\title{
Calendaring Extensions to WebDAV (CalDAV)
}

\section{Status of This Memo}

This document specifies an Internet standards track protocol for the Internet community, and requests discussion and suggestions for improvements. Please refer to the current edition of the "Internet Official Protocol Standards" (STD 1) for the standardization state and status of this protocol. Distribution of this memo is unlimited.

\section{Copyright Notice}

Copyright (C) The IETF Trust (2007). All Rights Reserved.

\begin{abstract}
This document defines extensions to the Web Distributed Authoring and Versioning (WebDAV) protocol to specify a standard way of accessing, managing, and sharing calendaring and scheduling information based on the iCalendar format. This document defines the "calendar-access" feature of CalDAV.
\end{abstract}




\section{Table of Contents}

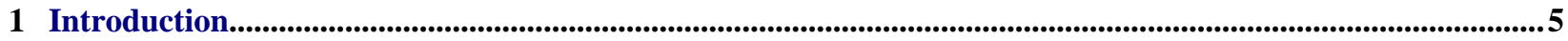

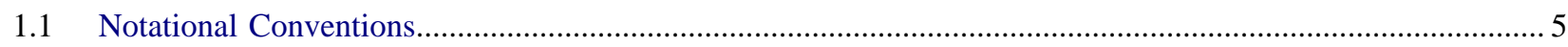

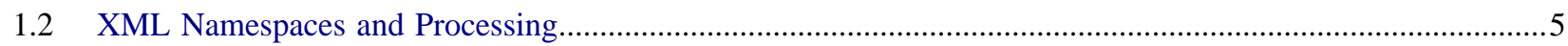

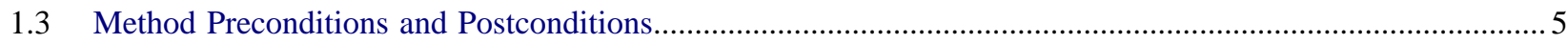

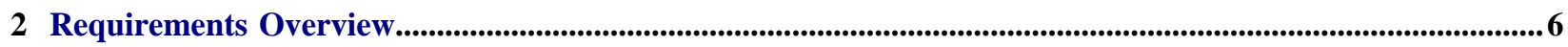

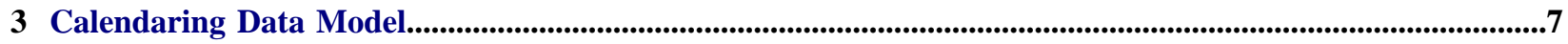

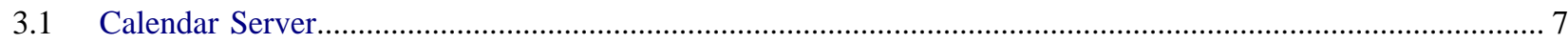

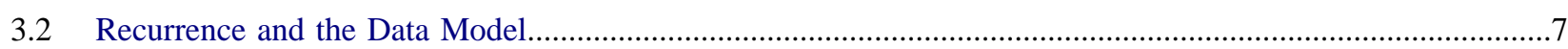

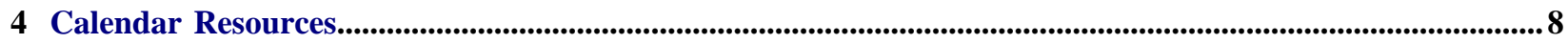

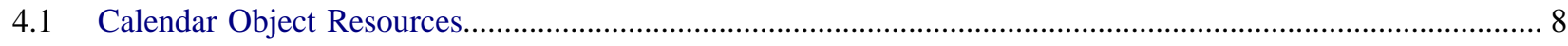

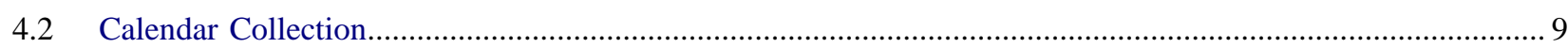

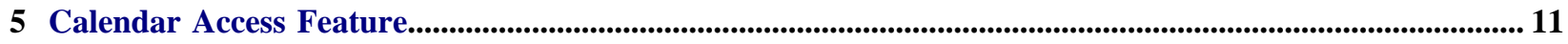

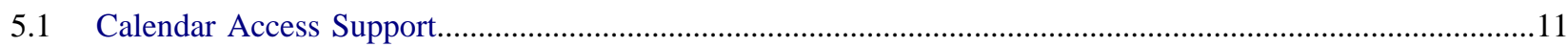

5.1.1 Example: Using OPTIONS for the Discovery of Calendar Access Support.......................................... 11

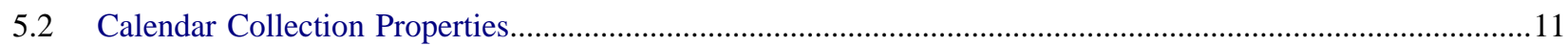

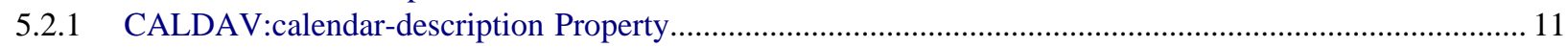

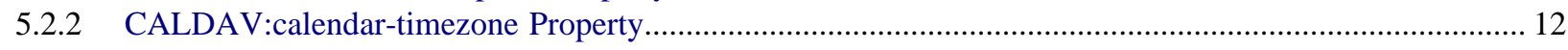

5.2.3 CALDAV:supported-calendar-component-set Property.................................................................. 13

5.2.4 CALDAV:supported-calendar-data Property.................................................................................. 14

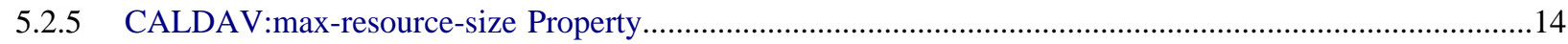

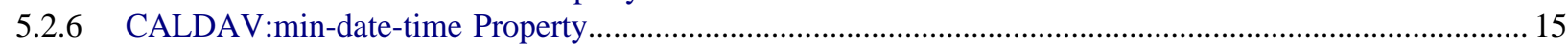

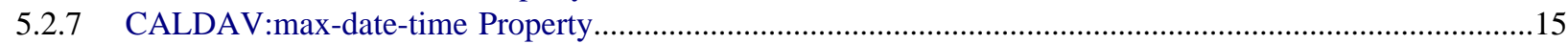

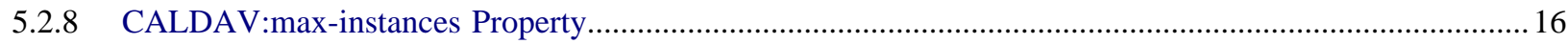

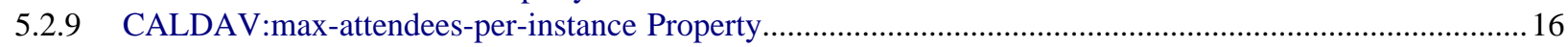

5.2.10 Additional Precondition for PROPPATCH....................................................................................

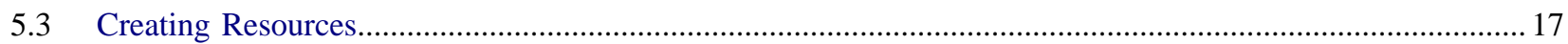

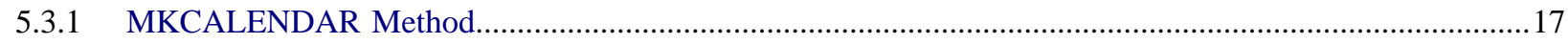

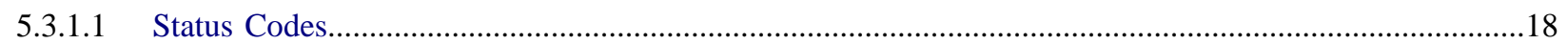

5.3.1.2 Example: Successful MKCALENDAR Request.............................................................................. 18

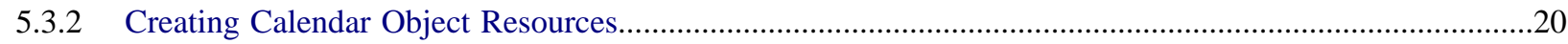

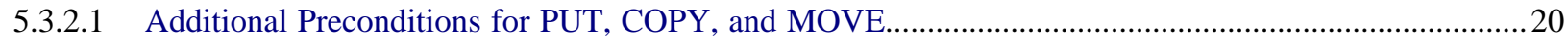

5.3.3 Non-Standard Components, Properties, and Parameters.................................................................. 21

5.3.4 Calendar Object Resource Entity Tag ...................................................................................22

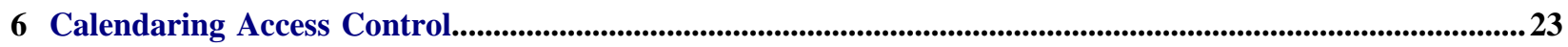

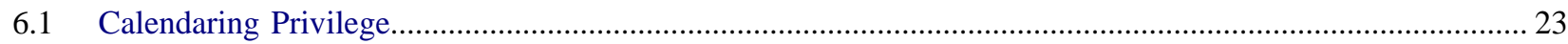

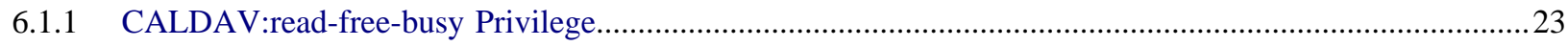

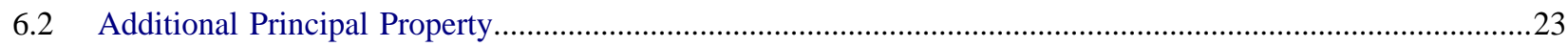

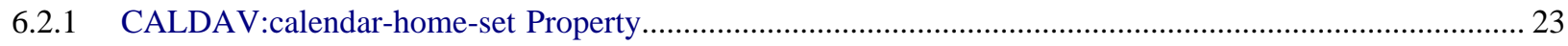

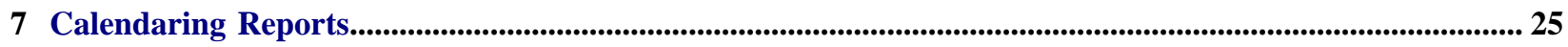

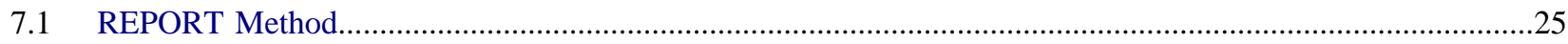




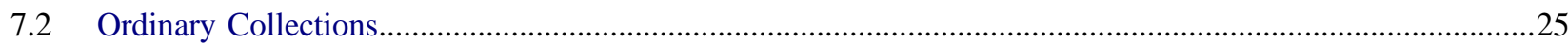

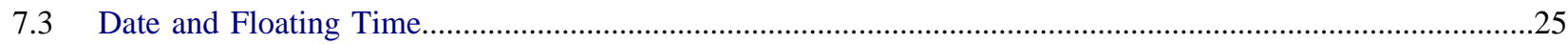

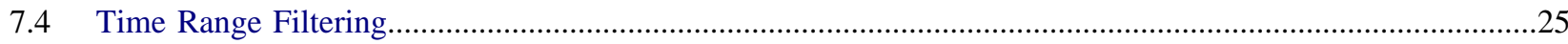

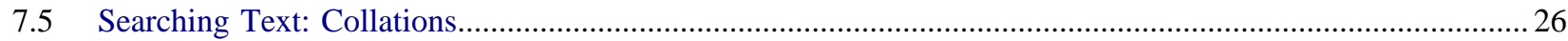

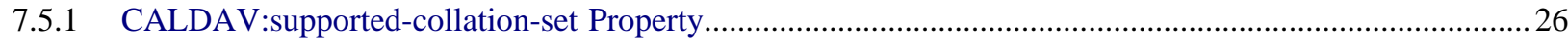

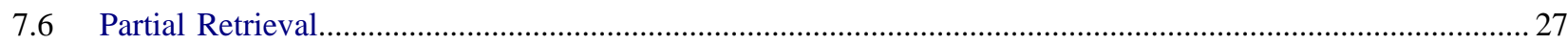

7.7 Non-Standard Components, Properties, and Parameters.......................................................................... 27

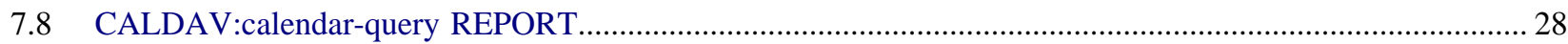

7.8.1 Example: Partial Retrieval of Events by Time Range.........................................................................29

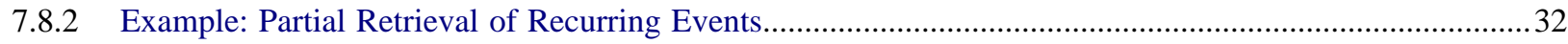

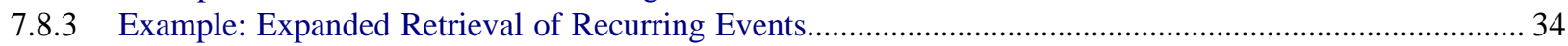

7.8.4 Example: Partial Retrieval of Stored Free Busy Components..................................................................36

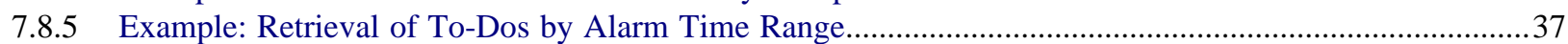

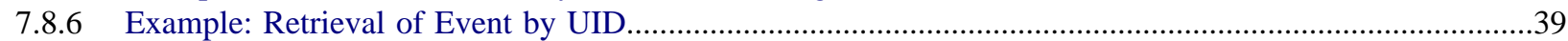

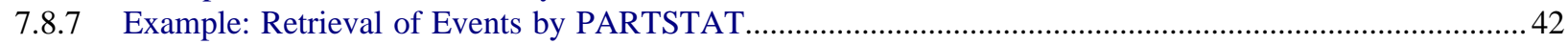

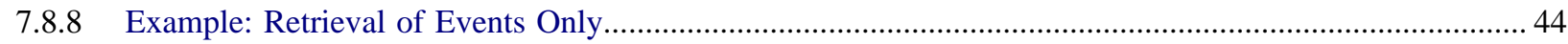

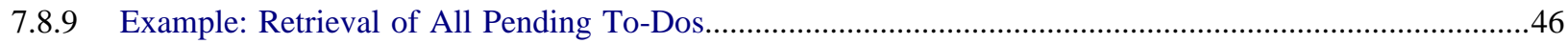

7.8.10 Example: Attempt to Query Unsupported Property.................................................................................48

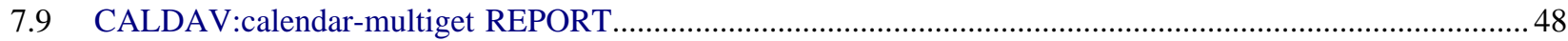

7.9.1 Example: Successful CALDAV:calendar-multiget REPORT................................................................. 49

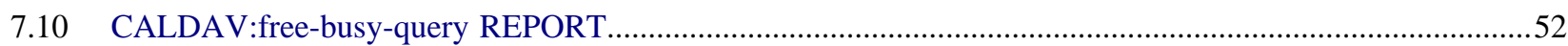

7.10.1 Example: Successful CALDAV:free-busy-query REPORT...............................................................53

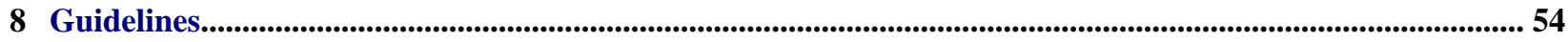

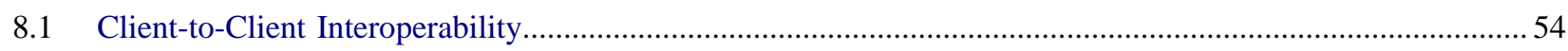

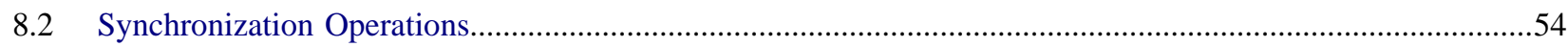

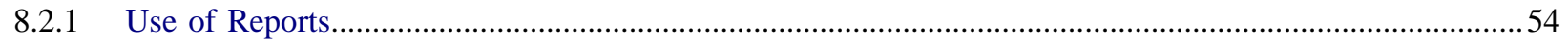

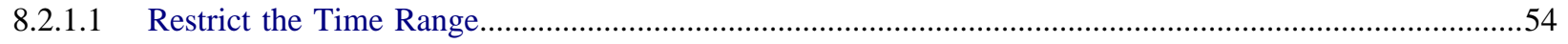

8.2.1.2 Synchronize by Time Range

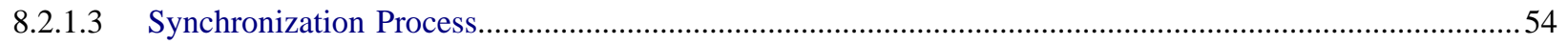

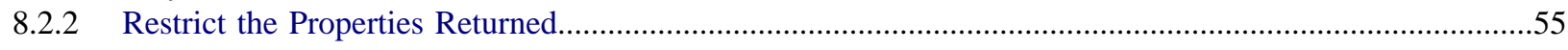

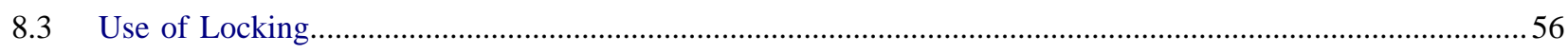

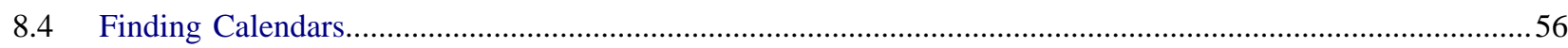

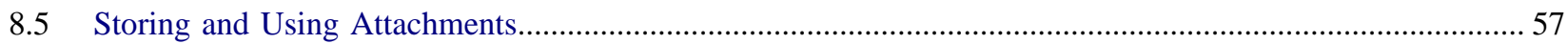

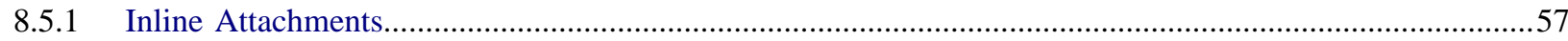

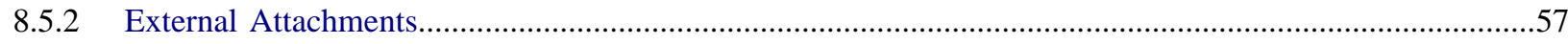

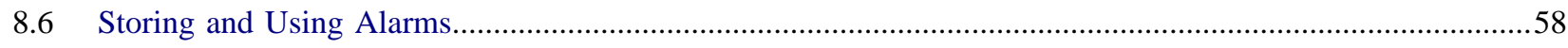

9 XML Element Definitions........................................................................................................................................59

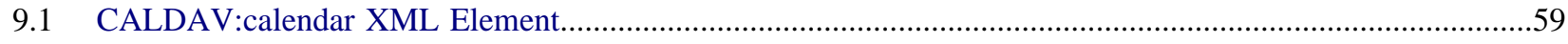

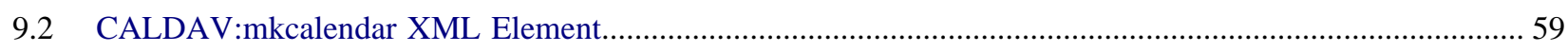

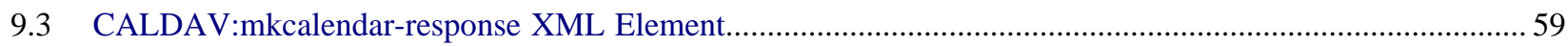

9.4 CALDAV:supported-collation XML Element........................................................................................5

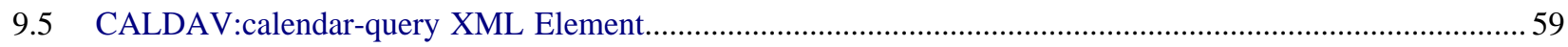

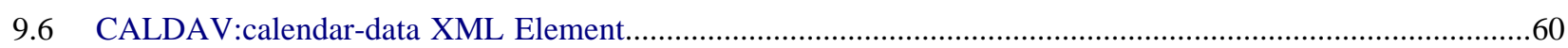

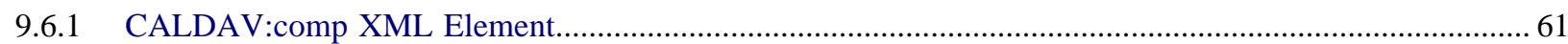




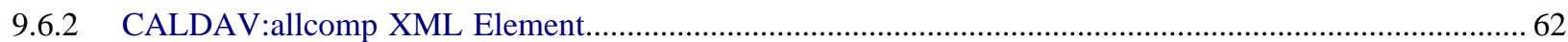

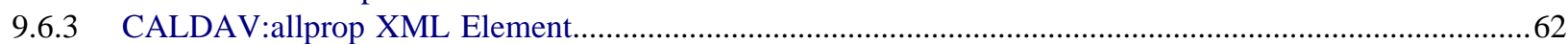

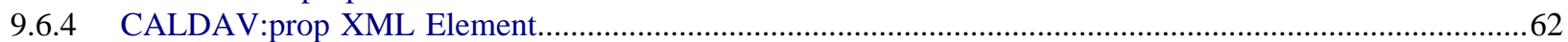

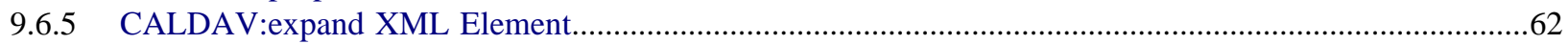

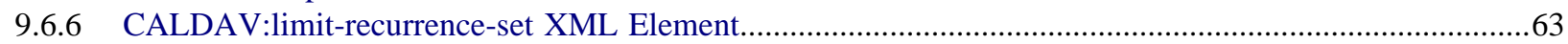

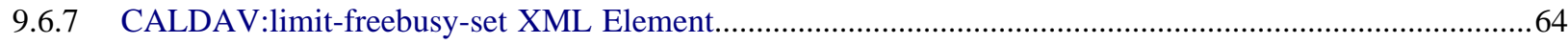

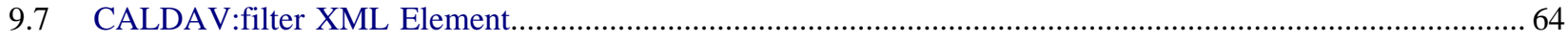

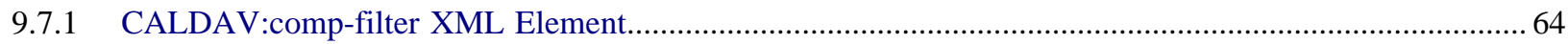

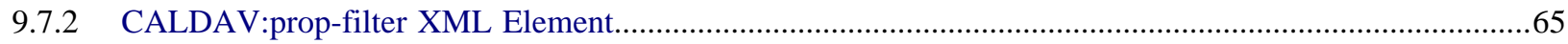

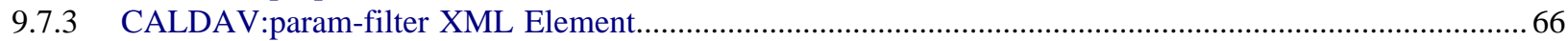

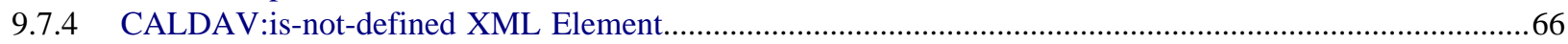

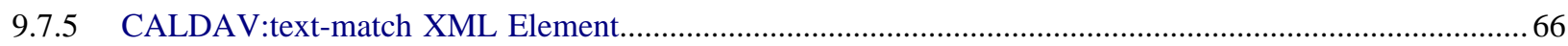

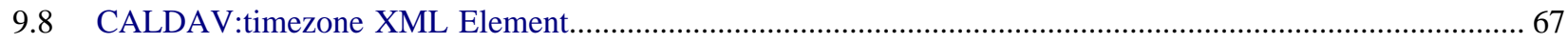

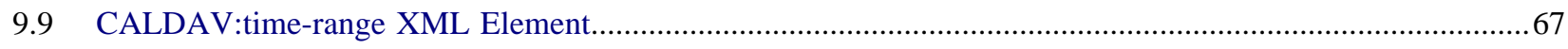

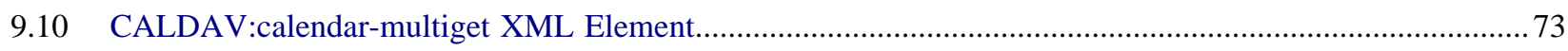

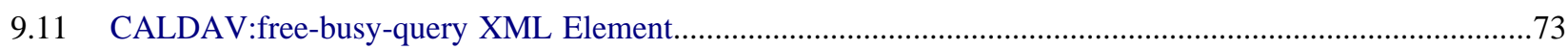

10 Internationalization Considerations........................................................................................................................... 74

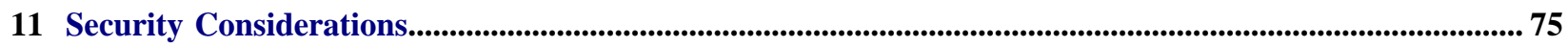

12 IANA Considerations....................................................................................................................................................76

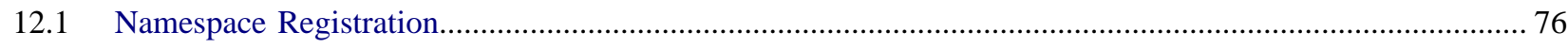

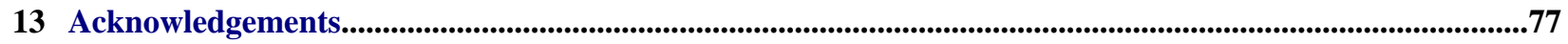

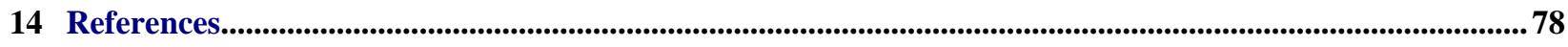

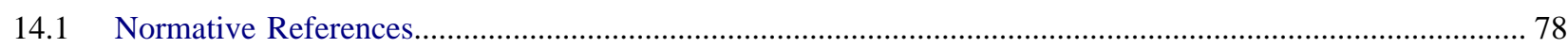

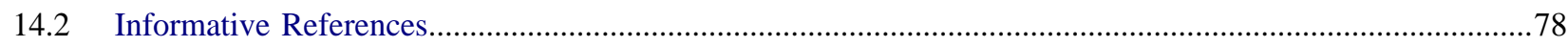

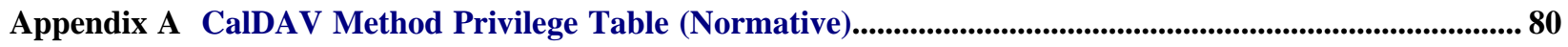

Appendix B Calendar Collections Used in the Examples............................................................................81

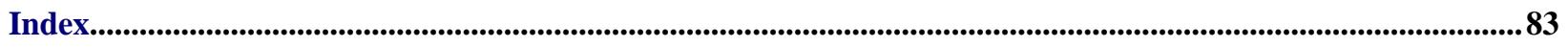

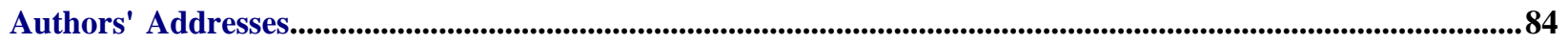

Intellectual Property and Copyright Statements...........................................................................................................84 


\section{Introduction}

The concept of using HTTP [RFC2616] and WebDAV [RFC2518] as a basis for a calendar access protocol is by no means a new concept: it was discussed in the IETF CALSCH working group as early as 1997 or 1998. Several companies have implemented calendar access protocols using HTTP to upload and download iCalendar [RFC2445] objects, and using WebDAV to get listings of resources. However, those implementations do not interoperate because there are many small and big decisions to be made in how to model calendaring data as WebDAV resources, as well as how to implement required features that aren't already part of WebDAV. This document proposes a way to model calendar data in WebDAV, with additional features to make an interoperable calendar access protocol.

\subsection{Notational Conventions}

The key words "MUST", "MUST NOT", "REQUIRED", "SHALL", "SHALL NOT", "SHOULD", "SHOULD NOT", "RECOMMENDED", "MAY", and "OPTIONAL" in this document are to be interpreted as described in [RFC2119].

The term "protected" is used in the Conformance field of property definitions as defined in Section 1.4.2 of [RFC3253].

When XML element types in the namespaces "DAV:" and "urn:ietf:params:xml:ns:caldav" are referenced in this document outside of the context of an XML fragment, the string "DAV:" and "CALDAV:" will be prefixed to the element type names, respectively.

\subsection{XML Namespaces and Processing}

Definitions of XML elements in this document use XML element type declarations (as found in XML Document Type Declarations), described in Section 3.2 of [W3C.REC-xml-20060816].

The namespace "urn:ietf:params:xml:ns:caldav" is reserved for the XML elements defined in this specification, its revisions, and related CalDAV specifications. XML elements defined by individual implementations MUST NOT use the "urn:ietf:params:xml:ns:caldav" namespace, and instead should use a namespace that they control.

The XML declarations used in this document do not include namespace information. Thus, implementers must not use these declarations as the only way to create valid CalDAV properties or to validate CalDAV XML element types. Some of the declarations refer to XML elements defined by WebDAV [RFC2518], which use the "DAV:" namespace. Wherever such XML elements appear, they are explicitly prefixed with "DAV:" to avoid confusion.

Also note that some CalDAV XML element names are identical to WebDAV XML element names, though their namespace differs. Care must be taken not to confuse the two sets of names.

Processing of XML by CalDAV clients and servers MUST follow the rules described in [RFC2518]; in particular, Section 14, and Appendix 3 of that specification.

\subsection{Method Preconditions and Postconditions}

A "precondition" of a method describes the state of the server that must be true for that method to be performed. A "postcondition" of a method describes the state of the server that must be true after that method has been completed. If a method precondition or postcondition for a request is not satisfied, the response status of the request MUST either be 403 (Forbidden), if the request should not be repeated because it will always fail, or 409 (Conflict), if it is expected that the user might be able to resolve the conflict and resubmit the request.

In order to allow better client handling of 403 and 409 responses, a distinct XML element type is associated with each method precondition and postcondition of a request. When a particular precondition is not satisfied or a particular postcondition cannot be achieved, the appropriate XML element MUST be returned as the child of a top-level DAV:error element in the response body, unless otherwise negotiated by the request. 


\section{Requirements Overview}

This section lists what functionality is required of a CalDAV server. To advertise support for CalDAV, a server:

- MUST support iCalendar [RFC2445] as a media type for the calendar object resource format;

- MUST support WebDAV Class 1 [RFC2518] (note that [rfc2518bis] describes clarifications to [RFC2518] that aid interoperability);

- MUST support WebDAV ACL [RFC3744] with the additional privilege defined in Section 6.1 of this document;

- MUST support transport over TLS [RFC2246] as defined in [RFC2818] (note that [RFC2246] has been obsoleted by [RFC4346]);

- MUST support ETags [RFC2616] with additional requirements specified in Section 5.3.4 of this document;

- MUST support all calendaring reports defined in Section 7 of this document; and

- MUST advertise support on all calendar collections and calendar object resources for the calendaring reports in the DAV:supported-report-set property, as defined in Versioning Extensions to WebDAV [RFC3253].

In addition, a server:

- SHOULD support the MKCALENDAR method defined in Section 5.3.1 of this document. 


\section{Calendaring Data Model}

One of the features that has made WebDAV a successful protocol is its firm data model. This makes it a useful framework for other applications such as calendaring. This specification follows the same pattern by developing all features based on a well-described data model.

As a brief overview, a CalDAV calendar is modeled as a WebDAV collection with a defined structure; each calendar collection contains a number of resources representing calendar objects as its direct child resource. Each resource representing a calendar object (event, to-do, journal entry, or other calendar components) is called a "calendar object resource". Each calendar object resource and each calendar collection can be individually locked and have individual WebDAV properties. Requirements derived from this model are provided in Section 4.1 and Section 4.2.

\subsection{Calendar Server}

A CalDAV server is a calendaring-aware engine combined with a WebDAV repository. A WebDAV repository is a set of WebDAV collections, containing other WebDAV resources, within a unified URL namespace. For example, the repository "http://www.example.com/webdav/" may contain WebDAV collections and resources, all of which have URLs beginning with "http://www.example.com/webdav/". Note that the root URL, "http:// www.example.com/", may not itself be a WebDAV repository (for example, if the WebDAV support is implemented through a servlet or other Web server extension).

A WebDAV repository MAY include calendar data in some parts of its URL namespace, and non-calendaring data in other parts.

A WebDAV repository can advertise itself as a CalDAV server if it supports the functionality defined in this specification at any point within the root of the repository. That might mean that calendaring data is spread throughout the repository and mixed with non-calendar data in nearby collections (e.g., calendar data may be found in /home/lisa/calendars/ as well as in /home/bernard/calendars/, and non-calendar data in /home/lisa/ contacts/). Or, it might mean that calendar data can be found only in certain sections of the repository (e.g., / calendar/). Calendaring features are only required in the repository sections that are or contain calendar object resources. Therefore, a repository confining calendar data to the /calendar/ collection would only need to support the CalDAV required features within that collection.

The CalDAV server or repository is the canonical location for calendar data and state information. Clients may submit requests to change data or download data. Clients may store calendar objects offline and attempt to synchronize at a later time. However, clients MUST be prepared for calendar data on the server to change between the time of last synchronization and when attempting an update, as calendar collections may be shared and accessible via multiple clients. Entity tags and other features make this possible.

\subsection{Recurrence and the Data Model}

Recurrence is an important part of the data model because it governs how many resources are expected to exist. This specification models a recurring calendar component and its recurrence exceptions as a single resource. In this model, recurrence rules, recurrence dates, exception rules, and exception dates are all part of the data in a single calendar object resource. This model avoids problems of limiting how many recurrence instances to store in the repository, how to keep recurrence instances in sync with the recurring calendar component, and how to link recurrence exceptions with the recurring calendar component. It also results in less data to synchronize between client and server, and makes it easier to make changes to all recurrence instances or to a recurrence rule. It makes it easier to create a recurring calendar component and to delete all recurrence instances.

Clients are not forced to retrieve information about all recurrence instances of a recurring component. The CALDAV:calendar-query and CALDAV:calendar-multiget reports defined in this document allow clients to retrieve only recurrence instances that overlap a given time range. 


\section{Calendar Resources}

\subsection{Calendar Object Resources}

Calendar object resources contained in calendar collections MUST NOT contain more than one type of calendar component (e.g., VEVENT, VTODO, VJOURNAL, VFREEBUSY, etc.) with the exception of VTIMEZONE components, which MUST be specified for each unique TZID parameter value specified in the iCalendar object. For instance, a calendar object resource can contain one VEVENT component and one VTIMEZONE component, but it cannot contain one VEVENT component and one VTODO component. Instead, the VEVENT and VTODO components would have to be stored in separate calendar object resources in the same collection.

Calendar object resources contained in calendar collections MUST NOT specify the iCalendar METHOD property.

The UID property value of the calendar components contained in a calendar object resource MUST be unique in the scope of the calendar collection in which they are stored.

Calendar components in a calendar collection that have different UID property values MUST be stored in separate calendar object resources.

Calendar components with the same UID property value, in a given calendar collection, MUST be contained in the same calendar object resource. This ensures that all components in a recurrence "set" are contained in the same calendar object resource. It is possible for a calendar object resource to just contain components that represent "overridden" instances (ones that modify the behavior of a regular instance, and thus include a RECURRENCE-ID property) without also including the "master" recurring component (the one that defines the recurrence "set" and does not contain any RECURRENCE-ID property).

For example, given the following iCalendar object: 


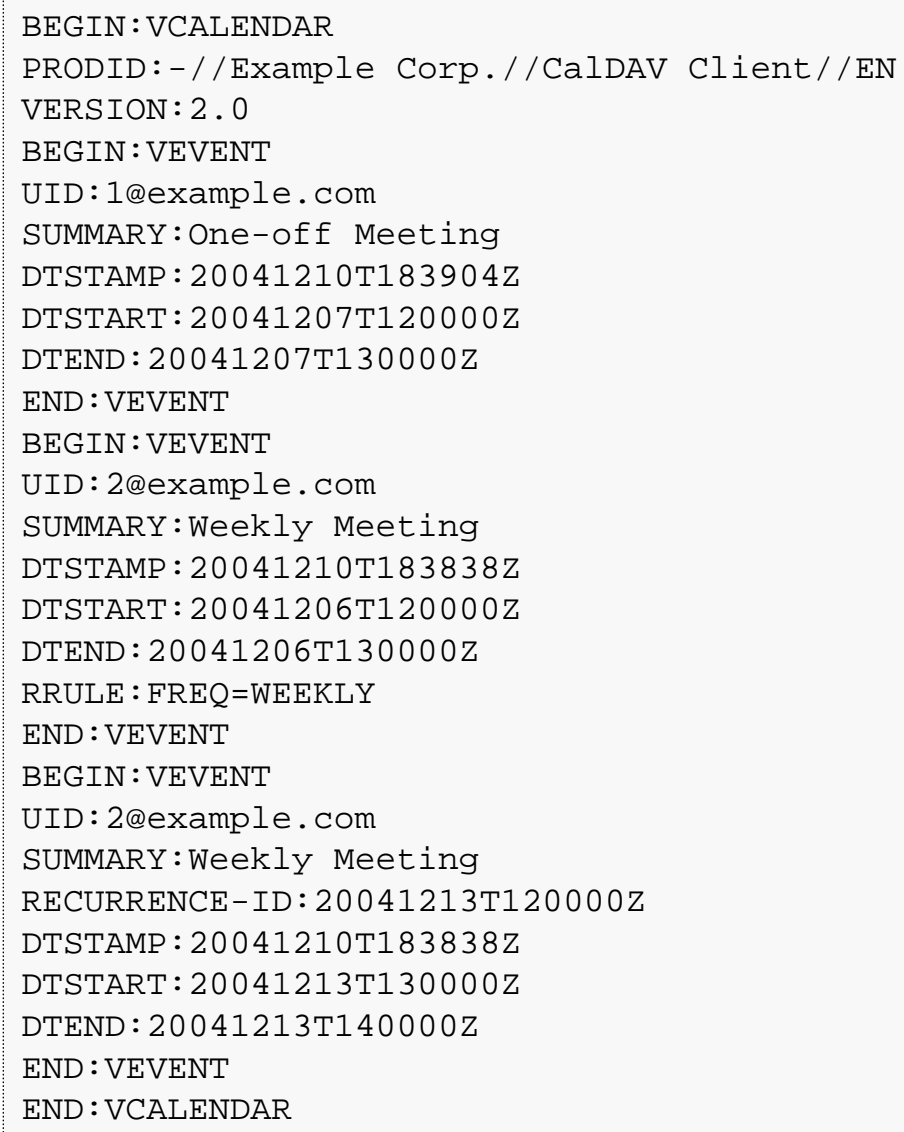

The VEVENT component with the UID value "1@example.com" would be stored in its own calendar object resource. The two VEVENT components with the UID value "2@example.com", which represent a recurring event where one recurrence instance has been overridden, would be stored in the same calendar object resource.

\subsection{Calendar Collection}

A calendar collection contains calendar object resources that represent calendar components within a calendar. A calendar collection is manifested to clients as a WebDAV resource collection identified by a URL. A calendar collection MUST report the DAV:collection and CALDAV:calendar XML elements in the value of the DAV:resourcetype property. The element type declaration for CALDAV:calendar is:

$<$ !ELEMENT calendar EMPTY>

A calendar collection can be created through provisioning (i.e., automatically created when a user's account is provisioned), or it can be created with the MKCALENDAR method (see Section 5.3.1). This method can be useful for a user to create additional calendars (e.g., soccer schedule) or for users to share a calendar (e.g., team events or conference rooms). However, note that this document doesn't define the purpose of extra calendar collections. Users must rely on non-standard cues to find out what a calendar collection is for, or use the CALDAV:calendar-description property defined in Section 5.2.1 to provide such a cue.

The following restrictions are applied to the resources within a calendar collection:

a. Calendar collections MUST only contain calendar object resources and collections that are not calendar collections, i.e., the only "top-level" non-collection resources allowed in a calendar collection are calendar object resources. This ensures that calendar clients do not have to deal with non-calendar data in a calendar collection, though they do have to distinguish between calendar object resources and collections when using standard WebDAV techniques to examine the contents of a collection. 
b. Collections contained in calendar collections MUST NOT contain calendar collections at any depth, i.e., "nesting" of calendar collections within other calendar collections at any depth is not allowed. This specification does not define how collections contained in a calendar collection are used or how they relate to any calendar object resources contained in the calendar collection.

Multiple calendar collections MAY be children of the same collection. 


\section{Calendar Access Feature}

\subsection{Calendar Access Support}

A server supporting the features described in this document MUST include "calendar-access" as a field in the DAV response header from an OPTIONS request on any resource that supports any calendar properties, reports, method, or privilege. A value of "calendar-access" in the DAV response header MUST indicate that the server supports all MUST level requirements specified in this document.

\subsubsection{Example: Using OPTIONS for the Discovery of Calendar Access Support}

$>$ Request $<<$

OPTIONS /home/bernard/calendars/ HTTP/1.1

Host: cal.example.com

$>>$ Response $<<$

HTTP/1.1 200 OK

Allow: OPTIONS, GET, HEAD, POST, PUT, DELETE, TRACE, COPY, MOVE

Allow: PROPFIND, PROPPATCH, LOCK, UNLOCK, REPORT, ACL

DAV: 1, 2, access-control, calendar-access

Date: Sat, 11 Nov 2006 09:32:12 GMT

Content-Length: 0

In this example, the OPTIONS method returns the value "calendar-access" in the DAV response header to indicate that the collection "/home/bernard/calendars/" supports the properties, reports, method, or privilege defined in this specification.

\subsection{Calendar Collection Properties}

This section defines properties for calendar collections.

\subsubsection{CALDAV:calendar-description Property}

Name: $\quad$ calendar-description

Namespace: urn:ietf:params:xml:ns:caldav

Purpose: Provides a human-readable description of the calendar collection.

Conformance: This property MAY be defined on any calendar collection. If defined, it MAY be protected and SHOULD NOT be returned by a PROPFIND DAV:allprop request (as defined in Section 12.14.1 of [RFC2518]). An xml:lang attribute indicating the human language of the description SHOULD be set for this property by clients or through server provisioning. Servers MUST return any xml:lang attribute if set for the property.

Description: If present, the property contains a description of the calendar collection that is suitable for presentation to a user. If not present, the client should assume no description for the calendar collection.

Definition:

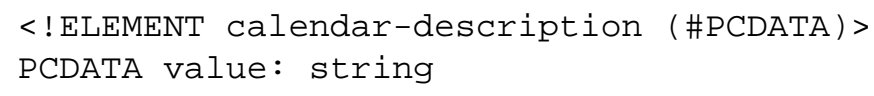


Example:

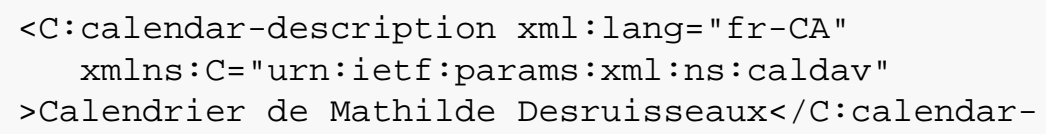

\subsubsection{CALDAV:calendar-timezone Property}

Name:

calendar-timezone

Namespace:

urn:ietf:params:xml:ns:caldav

Purpose:

Specifies a time zone on a calendar collection.

Conformance: This property SHOULD be defined on all calendar collections. If defined, it SHOULD NOT be returned by a PROPFIND DAV:allprop request (as defined in Section 12.14.1 of [RFC2518]).

Description: The CALDAV:calendar-timezone property is used to specify the time zone the server should rely on to resolve "date" values and "date with local time" values (i.e., floating time) to "date with UTC time" values. The server will require this information to determine if a calendar component scheduled with "date" values or "date with local time" values overlaps a CALDAV:time-range specified in a CALDAV:calendar-query REPORT. The server will also require this information to compute the proper FREEBUSY time period as "date with UTC time" in the VFREEBUSY component returned in a response to a CALDAV:free-busy-query REPORT request that takes into account calendar components scheduled with "date" values or "date with local time" values. In the absence of this property, the server MAY rely on the time zone of their choice.

Note: $\quad$ The iCalendar data embedded within the CALDAV:calendar-timezone XML element MUST follow the standard XML character data encoding rules, including use of \&lt;, \&gt; \&amp; etc. entity encoding or the use of a $<$ ! [CDATA $\ldots$... ]]> construct. In the later case, the iCalendar data cannot contain the character sequence "]]>", which is the end delimiter for the CDATA section.

Definition:

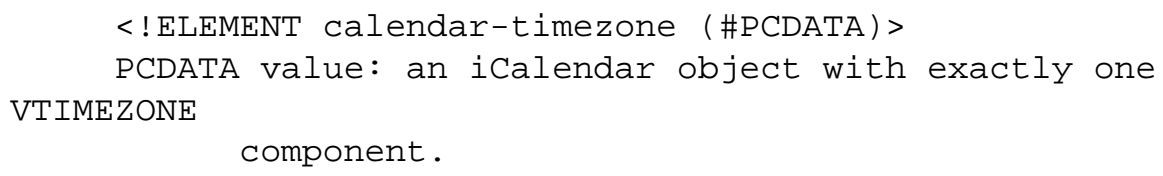




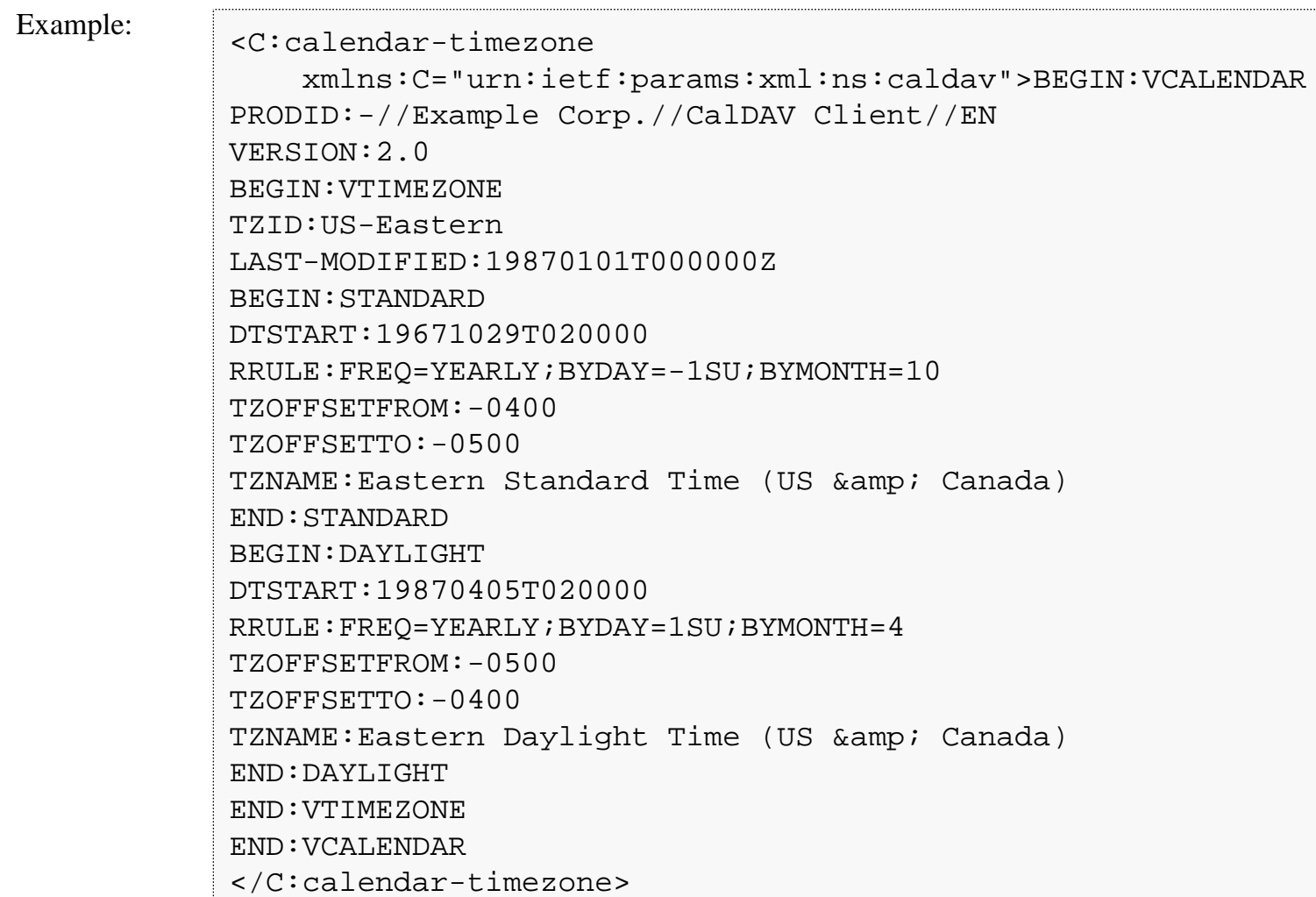

\subsubsection{CALDAV:supported-calendar-component-set Property}

Name: $\quad$ supported-calendar-component-set

Namespace: urn:ietf:params:xml:ns:caldav

Purpose: $\quad$ Specifies the calendar component types (e.g., VEVENT, VTODO, etc.) that calendar object resources can contain in the calendar collection.

Conformance: This property MAY be defined on any calendar collection. If defined, it MUST be protected and SHOULD NOT be returned by a PROPFIND DAV:allprop request (as defined in Section 12.14.1 of [RFC2518]).

Description: The CALDAV:supported-calendar-component-set property is used to specify restrictions on the calendar component types that calendar object resources may contain in a calendar collection. Any attempt by the client to store calendar object resources with component types not listed in this property, if it exists, MUST result in an error, with the CALDAV:supportedcalendar-component precondition (Section 5.3.2.1) being violated. Since this property is protected, it cannot be changed by clients using a PROPPATCH request. However, clients can initialize the value of this property when creating a new calendar collection with MKCALENDAR. The empty-element tag <C:comp name="VTIMEZONE"/> MUST only be specified if support for calendar object resources that only contain VTIMEZONE components is provided or desired. Support for VTIMEZONE components in calendar object resources that contain VEVENT or VTODO components is always assumed. In the absence of this property, the server MUST accept all component types, and the client can assume that all component types are accepted.

Definition:

$$
<\text { !ELEMENT supported-calendar-component-set (comp+)> }
$$


Example:

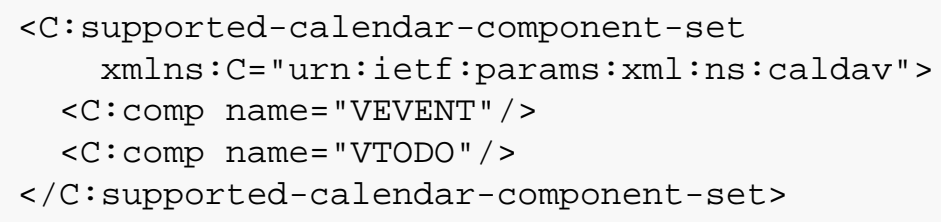

\subsubsection{CALDAV:supported-calendar-data Property}

Name: $\quad$ supported-calendar-data

Namespace: urn:ietf:params:xml:ns:caldav

Purpose: $\quad$ Specifies what media types are allowed for calendar object resources in a calendar collection.

Conformance: This property MAY be defined on any calendar collection. If defined, it MUST be protected and SHOULD NOT be returned by a PROPFIND DAV:allprop request (as defined in Section 12.14.1 of [RFC2518]).

Description: The CALDAV:supported-calendar-data property is used to specify the media type supported for the calendar object resources contained in a given calendar collection (e.g., iCalendar version 2.0). Any attempt by the client to store calendar object resources with a media type not listed in this property MUST result in an error, with the CALDAV:supported-calendardata precondition (Section 5.3.2.1) being violated. In the absence of this property, the server MUST only accept data with the media type "text/calendar" and iCalendar version 2.0, and clients can assume that the server will only accept this data.

Definition:

<!ELEMENT supported-calendar-data (calendar-data+)>

Example:

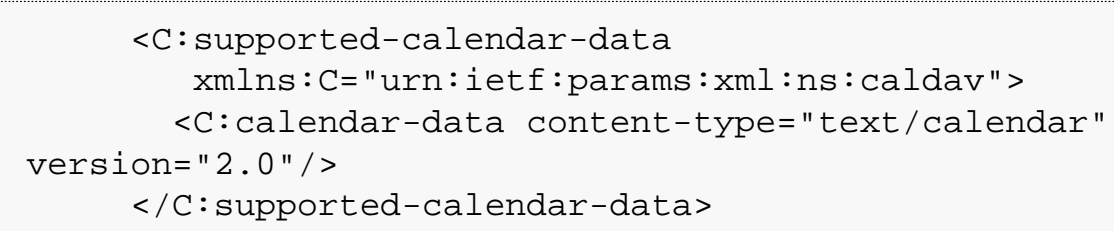

\subsubsection{CALDAV:max-resource-size Property}

Name:

Namespace:

Purpose:

Conformance:

Description:

Definition:

max-resource-size

urn:ietf:params:xml:ns:caldav

Provides a numeric value indicating the maximum size of a resource in octets that the server is willing to accept when a calendar object resource is stored in a calendar collection.

This property MAY be defined on any calendar collection. If defined, it MUST be protected and SHOULD NOT be returned by a PROPFIND DAV:allprop request (as defined in Section $\underline{12.14 .1}$ of [RFC2518]).

The CALDAV:max-resource-size is used to specify a numeric value that represents the maximum size in octets that the server is willing to accept when a calendar object resource is stored in a calendar collection. Any attempt to store a calendar object resource exceeding this size MUST result in an error, with the CALDAV:max-resource-size precondition (Section 5.3.2.1) being violated. In the absence of this property, the client can assume that the server will allow storing a resource of any reasonable size.

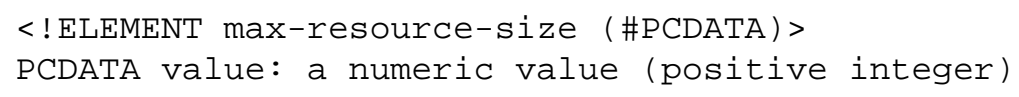


Example:

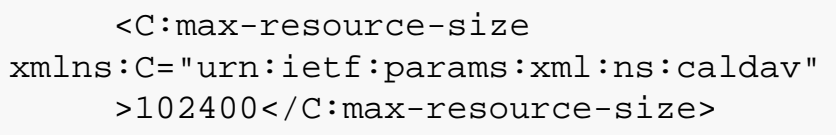

\subsubsection{CALDAV:min-date-time Property}

Name:

Namespace:

Purpose:

Conformance:

Description:

Definition:

Example:

min-date-time

$$
\text { urn:ietf:params:xml:ns:caldav }
$$

Provides a DATE-TIME value indicating the earliest date and time (in UTC) that the server is willing to accept for any DATE or DATE-TIME value in a calendar object resource stored in a calendar collection.

This property MAY be defined on any calendar collection. If defined, it MUST be protected and SHOULD NOT be returned by a PROPFIND DAV:allprop request (as defined in $\underline{\text { Section }}$ $\underline{12.14 .1}$ of [RFC2518]).

The CALDAV:min-date-time is used to specify an iCalendar DATE-TIME value in UTC that indicates the earliest inclusive date that the server is willing to accept for any explicit DATE or DATE-TIME value in a calendar object resource stored in a calendar collection. Any attempt to store a calendar object resource using a DATE or DATE-TIME value earlier than this value MUST result in an error, with the CALDAV:min-date-time precondition (Section 5.3.2.1) being violated. Note that servers MUST accept recurring components that specify instances beyond this limit, provided none of those instances have been overridden. In that case, the server MAY simply ignore those instances outside of the acceptable range when processing reports on the calendar object resource. In the absence of this property, the client can assume any valid iCalendar date may be used at least up to the CALDAV:max-date-time value, if that is defined.

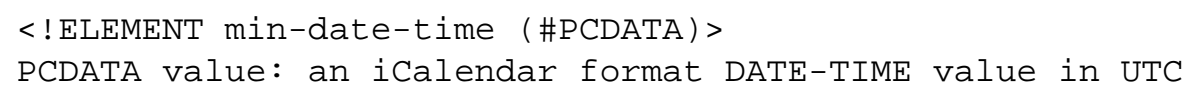

\subsubsection{CALDAV:max-date-time Property}

Name:

Namespace:

Purpose:

Conformance:

Description: max-date-time

urn:ietf:params:xml:ns:caldav

Provides a DATE-TIME value indicating the latest date and time (in UTC) that the server is willing to accept for any DATE or DATE-TIME value in a calendar object resource stored in a calendar collection.

This property MAY be defined on any calendar collection. If defined, it MUST be protected and SHOULD NOT be returned by a PROPFIND DAV:allprop request (as defined in Section $\underline{12.14 .1}$ of [RFC2518]).

The CALDAV:max-date-time is used to specify an iCalendar DATE-TIME value in UTC that indicates the inclusive latest date that the server is willing to accept for any date or time value in a calendar object resource stored in a calendar collection. Any attempt to store a calendar object resource using a DATE or DATE-TIME value later than this value MUST result in an error, with the CALDAV:max-date-time precondition (Section 5.3.2.1) being violated. Note that servers MUST accept recurring components that specify instances beyond this limit, provided none of those instances have been overridden. In that case, the server MAY simply 
ignore those instances outside of the acceptable range when processing reports on the calendar object resource. In the absence of this property, the client can assume any valid iCalendar date may be used at least down to the CALDAV:min-date-time value, if that is defined.

Definition: <!ELEMENT max-date-time (\#PCDATA) > PCDATA value: an iCalendar format DATE-TIME value in UTC

Example: $<$ : $m a x-d a t e-t i m e x m l n s: C=" u r n:$ iet $\mathrm{f}$ :params:xml:ns:caldav" $>20491231 \mathrm{~T} 235959 \mathrm{Z}</ \mathrm{C}$ :max-date-time>

\subsubsection{CALDAV:max-instances Property}

Name: max-instances

Namespace: urn:ietf:params:xml:ns:caldav

Purpose:

Conformance:

Provides a numeric value indicating the maximum number of recurrence instances that a calendar object resource stored in a calendar collection can generate.

Conformance:

This property MAY be defined on any calendar collection. If defined, it MUST be protected and SHOULD NOT be returned by a PROPFIND DAV:allprop request (as defined in $\underline{\text { Section }}$ $\underline{12.14 .1}$ of [RFC2518]).

Description:

The CALDAV:max-instances is used to specify a numeric value that indicates the maximum number of recurrence instances that a calendar object resource stored in a calendar collection can generate. Any attempt to store a calendar object resource with a recurrence pattern that generates more instances than this value MUST result in an error, with the CALDAV:maxinstances precondition (Section 5.3.2.1) being violated. In the absence of this property, the client can assume that the server has no limits on the number of recurrence instances it can handle or expand.

Definition:

$<$ !ELEMENT max-instances (\#PCDATA) > PCDATA value: a numeric value (integer greater than zero)

Example: $<$ C:max-instances xmlns:C="urn:ietf:params:xml:ns:caldav" $>100</ C:$ max-instances $>$

\subsubsection{CALDAV:max-attendees-per-instance Property}

Name:

Namespace:

Purpose:

Conformance:

Description: max-attendees-per-instance

urn:ietf:params:xml:ns:caldav

Provides a numeric value indicating the maximum number of ATTENDEE properties in any instance of a calendar object resource stored in a calendar collection.

This property MAY be defined on any calendar collection. If defined, it MUST be protected and SHOULD NOT be returned by a PROPFIND DAV:allprop request (as defined in $\underline{\text { Section }}$ $\underline{12.14 .1}$ of [RFC2518]).

The CALDAV:max-attendees-per-instance is used to specify a numeric value that indicates the maximum number of iCalendar ATTENDEE properties on any one instance of a calendar object resource stored in a calendar collection. Any attempt to store a calendar object resource with more ATTENDEE properties per instance than this value MUST result in an error, with the CALDAV:max-attendees-per-instance precondition (Section 5.3.2.1) being violated. In the absence of this property, the client can assume that the server can handle any number of ATTENDEE properties in a calendar component. 
Definition:

<!ELEMENT max-attendees-per-instance (\#PCDATA)>

PCDATA value: a numeric value (integer greater than zero)

Example:

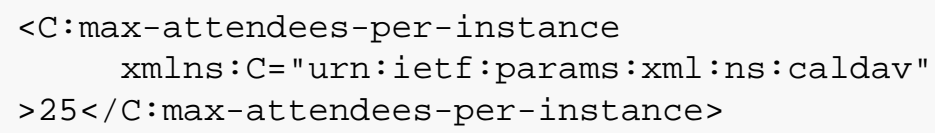

\subsubsection{Additional Precondition for PROPPATCH}

This specification requires an additional Precondition for the PROPPATCH method. The precondition is:

(CALDAV:valid-calendar-data): The time zone specified in CALDAV:calendar-timezone property MUST be a valid iCalendar object containing a single valid VTIMEZONE component.

\subsection{Creating Resources}

Calendar collections and calendar object resources may be created by either a CalDAV client or by the CalDAV server. This specification defines restrictions and a data model that both clients and servers MUST adhere to when manipulating such calendar data.

\subsubsection{MKCALENDAR Method}

An HTTP request using the MKCALENDAR method creates a new calendar collection resource. A server MAY restrict calendar collection creation to particular collections.

Support for MKCALENDAR on the server is only RECOMMENDED and not REQUIRED because some calendar stores only support one calendar per user (or principal), and those are typically pre-created for each account. However, servers and clients are strongly encouraged to support MKCALENDAR whenever possible to allow users to create multiple calendar collections to help organize their data better.

Clients SHOULD use the DAV:displayname property for a human-readable name of the calendar. Clients can either specify the value of the DAV:displayname property in the request body of the MKCALENDAR request, or alternatively issue a PROPPATCH request to change the DAV:displayname property to the appropriate value immediately after issuing the MKCALENDAR request. Clients SHOULD NOT set the DAV:displayname property to be the same as any other calendar collection at the same URI "level". When displaying calendar collections to users, clients SHOULD check the DAV:displayname property and use that value as the name of the calendar. In the event that the DAV:displayname property is empty, the client MAY use the last part of the calendar collection URI as the name; however, that path segment may be "opaque" and not represent any meaningful human-readable text.

If a MKCALENDAR request fails, the server state preceding the request MUST be restored.

\section{Marshalling:}

If a request body is included, it MUST be a CALDAV:mkcalendar XML element. Instruction processing MUST occur in the order instructions are received (i.e., from top to bottom). Instructions MUST either all be executed or none executed. Thus, if any error occurs during processing, all executed instructions MUST be undone and a proper error result returned. Instruction processing details can be found in the definition of the DAV:set instruction in Section 12.13.2 of [RFC2518].

$$
<\text { !ELEMENT mkcalendar (DAV:set) > }
$$

If a response body for a successful request is included, it MUST be a CALDAV:mkcalendar-response XML element.

$$
\text { <!ELEMENT mkCalendar-response ANY> }
$$

The response MUST include a Cache-Control:no-cache header. 


\section{Preconditions:}

(DAV:resource-must-be-null): A resource MUST NOT exist at the Request-URI;

(CALDAV:calendar-collection-location-ok): The Request-URI MUST identify a location where a calendar collection can be created;

(CALDAV:valid-calendar-data): The time zone specified in the CALDAV:calendar-timezone property MUST be a valid iCalendar object containing a single valid VTIMEZONE component;

(DAV:needs-privilege): The DAV:bind privilege MUST be granted to the current user on the parent collection of the Request-URI.

\section{Postconditions:}

(CALDAV:initialize-calendar-collection): A new calendar collection exists at the Request-URI. The DAV:resourcetype of the calendar collection MUST contain both DAV:collection and CALDAV:calendar XML elements.

\subsubsection{Status Codes}

The following are examples of response codes one would expect to get in a response to a MKCALENDAR request. Note that this list is by no means exhaustive.

201 (Created) - The calendar collection resource was created in its entirety;

207 (Multi-Status) - The calendar collection resource was not created since one or more DAV:set instructions specified in the request body could not be processed successfully. The following are examples of response codes one would expect to be used in a 207 (Multi-Status) response in this situation:

403 (Forbidden) - The client, for reasons the server chooses not to specify, cannot alter one of the properties;

409 (Conflict) - The client has provided a value whose semantics are not appropriate for the property. This includes trying to set read-only properties;

424 (Failed Dependency) - The DAV:set instruction on the specified resource would have succeeded if it were not for the failure of another DAV:set instruction specified in the request body;

423 (Locked) - The specified resource is locked and the client either is not a lock owner or the lock type requires a lock token to be submitted and the client did not submit it; and

507 (Insufficient Storage) - The server did not have sufficient space to record the property;

403 (Forbidden) - This indicates at least one of two conditions: 1) the server does not allow the creation of calendar collections at the given location in its namespace, or 2) the parent collection of the Request-URI exists but cannot accept members;

409 (Conflict) - A collection cannot be made at the Request-URI until one or more intermediate collections have been created;

415 (Unsupported Media Type) - The server does not support the request type of the body; and

507 (Insufficient Storage) - The resource does not have sufficient space to record the state of the resource after the execution of this method.

\subsubsection{Example: Successful MKCALENDAR Request}

This example creates a calendar collection called /home/lisa/calendars/events/ on the server cal.example.com with specific values for the properties DAV:displayname, CALDAV:calendar-description, CALDAV:supported-calendar-component-set, and CALDAV:calendar-timezone. 


\section{> Request $<<$}

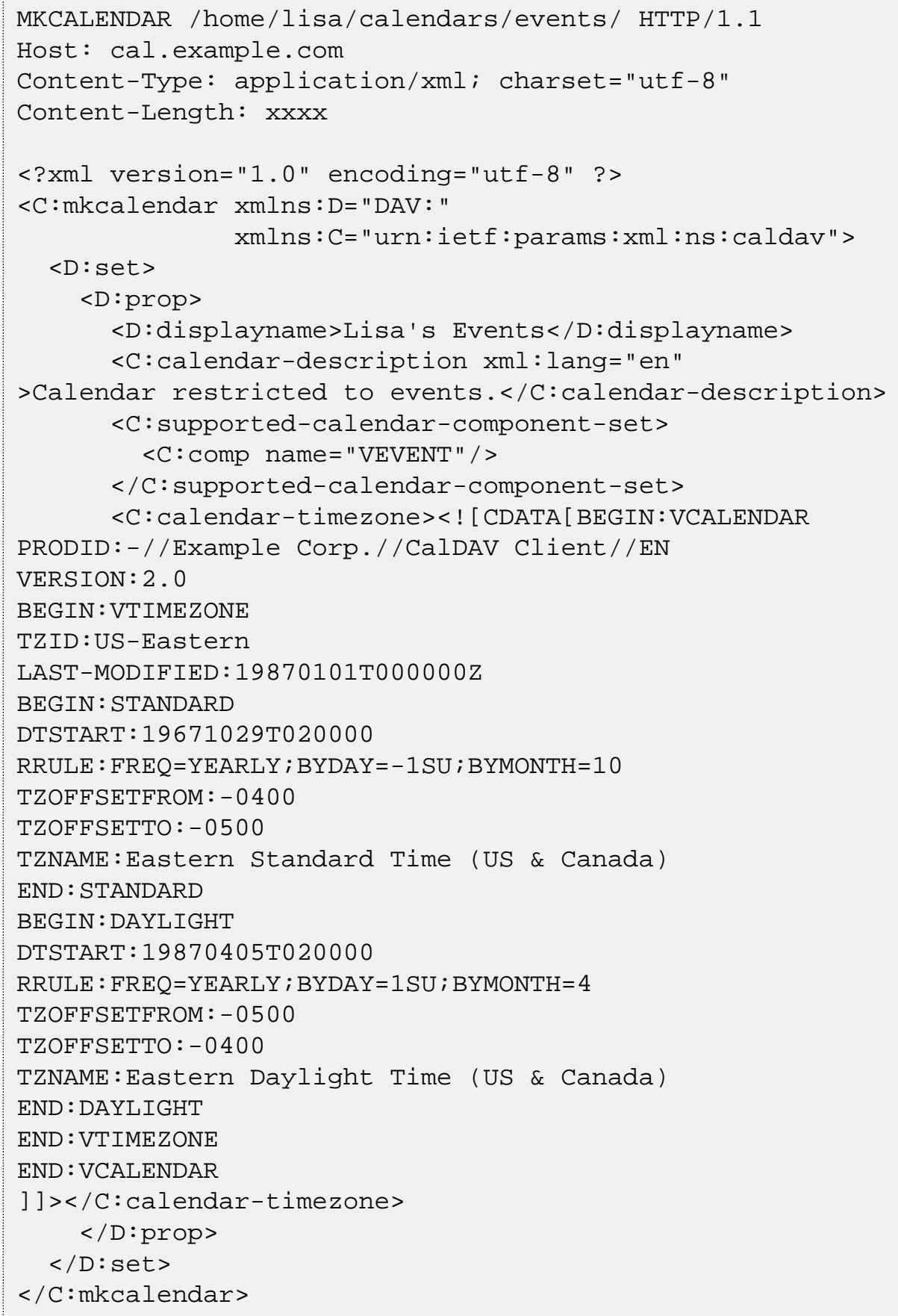

>> Response <<

HTTP/1.1 201 Created

Cache-Control: no-cache

Date: Sat, 11 Nov 2006 09:32:12 GMT

Content-Length: 0 


\subsubsection{Creating Calendar Object Resources}

Clients populate calendar collections with calendar object resources. The URL for each calendar object resource is entirely arbitrary and does not need to bear a specific relationship to the calendar object resource's iCalendar properties or other metadata. New calendar object resources MUST be created with a PUT request targeted at an unmapped URI. A PUT request targeted at a mapped URI updates an existing calendar object resource.

When servers create new resources, it's not hard for the server to choose an unmapped URI. It's slightly tougher for clients, because a client might not want to examine all resources in the collection and might not want to lock the entire collection to ensure that a new resource isn't created with a name collision. However, there is an HTTP feature to mitigate this. If the client intends to create a new non-collection resource, such as a new VEVENT, the client SHOULD use the HTTP request header "If-None-Match: *" on the PUT request. The Request-URI on the PUT request MUST include the target collection, where the resource is to be created, plus the name of the resource in the last path segment. The "If-None-Match: *" request header ensures that the client will not inadvertently overwrite an existing resource if the last path segment turned out to already be used.

\section{$>$ Request $<<$}

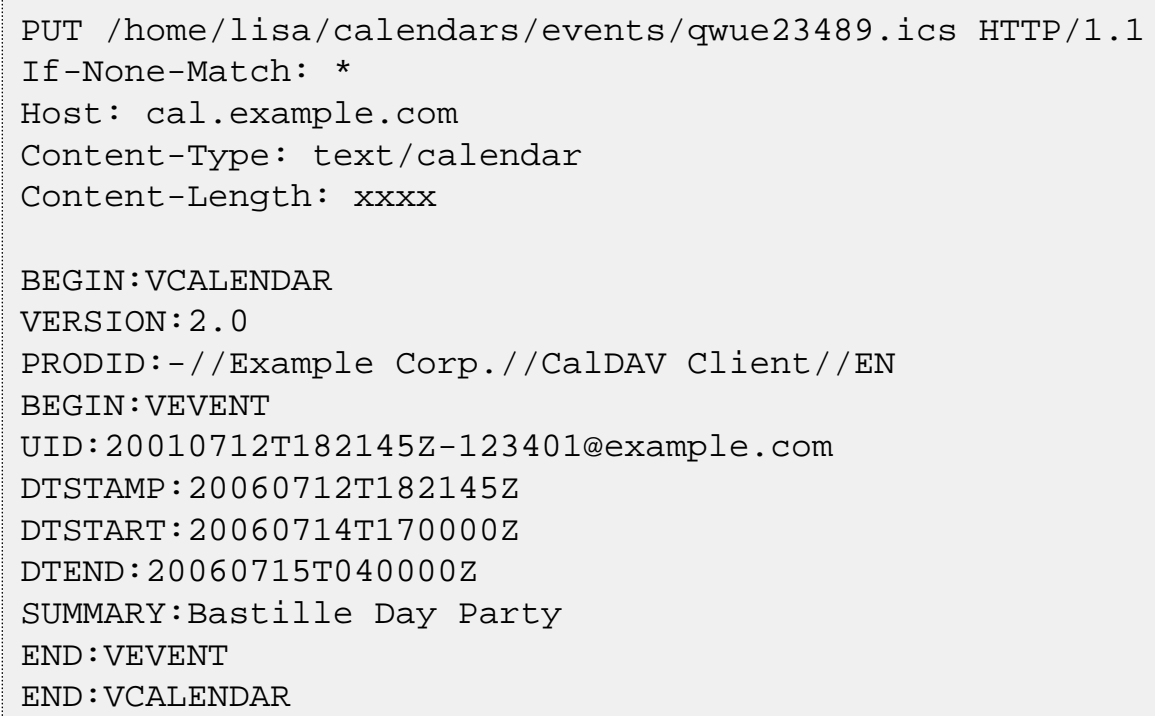

The request to change an existing event is the same, but with a specific ETag in the "If-Match" header, rather than the "If-None-Match" header.

As indicated in Section 3.10 of [RFC2445], the URL of calendar object resources containing (an arbitrary set of) calendaring and scheduling information may be suffixed by ".ics", and the URL of calendar object resources containing free or busy time information may be suffixed by ".ifb".

\subsubsection{Additional Preconditions for PUT, COPY, and MOVE}

This specification creates additional Preconditions for PUT, COPY, and MOVE methods. These preconditions apply when a PUT operation of a calendar object resource into a calendar collection occurs, or when a COPY or MOVE operation of a calendar object resource into a calendar collection occurs, or when a COPY or MOVE operation occurs on a calendar collection. 
The new preconditions are:

(CALDAV:supported-calendar-data): The resource submitted in the PUT request, or targeted by a COPY or MOVE request, MUST be a supported media type (i.e., iCalendar) for calendar object resources;

(CALDAV:valid-calendar-data): The resource submitted in the PUT request, or targeted by a COPY or MOVE request, MUST be valid data for the media type being specified (i.e., MUST contain valid iCalendar data);

(CALDAV:valid-calendar-object-resource): The resource submitted in the PUT request, or targeted by a COPY or MOVE request, MUST obey all restrictions specified in Section 4.1 (e.g., calendar object resources MUST NOT contain more than one type of calendar component, calendar object resources MUST NOT specify the iCalendar METHOD property, etc.);

(CALDAV:supported-calendar-component): The resource submitted in the PUT request, or targeted by a COPY or MOVE request, MUST contain a type of calendar component that is supported in the targeted calendar collection;

(CALDAV:no-uid-conflict): The resource submitted in the PUT request, or targeted by a COPY or MOVE request, MUST NOT specify an iCalendar UID property value already in use in the targeted calendar collection or overwrite an existing calendar object resource with one that has a different UID property value. Servers SHOULD report the URL of the resource that is already making use of the same UID property value in the DAV:href element;

<!ELEMENT no-uid-conflict (DAV:href) >

(CALDAV:calendar-collection-location-ok): In a COPY or MOVE request, when the Request-URI is a calendar collection, the Destination-URI MUST identify a location where a calendar collection can be created;

(CALDAV:max-resource-size): The resource submitted in the PUT request, or targeted by a COPY or MOVE request, MUST have an octet size less than or equal to the value of the CALDAV:max-resourcesize property value (Section 5.2.5) on the calendar collection where the resource will be stored;

(CALDAV:min-date-time): The resource submitted in the PUT request, or targeted by a COPY or MOVE request, MUST have all of its iCalendar DATE or DATE-TIME property values (for each recurring instance) greater than or equal to the value of the CALDAV:min-date-time property value (Section 5.2.6) on the calendar collection where the resource will be stored;

(CALDAV:max-date-time): The resource submitted in the PUT request, or targeted by a COPY or MOVE request, MUST have all of its iCalendar DATE or DATE-TIME property values (for each recurring instance) less than the value of the CALDAV:max-date-time property value (Section 5.2.7) on the calendar collection where the resource will be stored;

(CALDAV:max-instances): The resource submitted in the PUT request, or targeted by a COPY or MOVE request, MUST generate a number of recurring instances less than or equal to the value of the CALDAV:max-instances property value (Section 5.2.8) on the calendar collection where the resource will be stored;

(CALDAV:max-attendees-per-instance): The resource submitted in the PUT request, or targeted by a COPY or MOVE request, MUST have a number of ATTENDEE properties on any one instance less than or equal to the value of the CALDAV:max-attendees-per-instance property value (Section 5.2.9) on the calendar collection where the resource will be stored;

\subsubsection{Non-Standard Components, Properties, and Parameters}

iCalendar provides a "standard mechanism for doing non-standard things". This extension support allows implementers to make use of non-standard components, properties, and parameters whose names are prefixed with the text "X-".

Servers MUST support the use of non-standard components, properties, and parameters in calendar object resources stored via the PUT method. 
Servers may need to enforce rules for their own "private" components, properties, or parameters, so servers MAY reject any attempt by the client to change those or use values for those outside of any restrictions the server may have. Servers SHOULD ensure that any "private" components, properties, or parameters it uses follow the convention of including a vendor id in the "X-" name, as described in Section 4.2 of [RFC2445], e.g., "X-ABC-PRIVATE".

\subsubsection{Calendar Object Resource Entity Tag}

The DAV:getetag property MUST be defined and set to a strong entity tag on all calendar object resources.

A response to a GET request targeted at a calendar object resource MUST contain an ETag response header field indicating the current value of the strong entity tag of the calendar object resource.

Servers SHOULD return a strong entity tag (ETag header) in a PUT response when the stored calendar object resource is equivalent by octet equality to the calendar object resource submitted in the body of the PUT request. This allows clients to reliably use the returned strong entity tag for data synchronization purposes. For instance, the client can do a PROPFIND request on the stored calendar object resource and have the DAV:getetag property returned, and compare that value with the strong entity tag it received on the PUT response, and know that if they are equal, then the calendar object resource on the server has not been changed.

In the case where the data stored by a server as a result of a PUT request is not equivalent by octet equality to the submitted calendar object resource, the behavior of the ETag response header is not specified here, with the exception that a strong entity tag MUST NOT be returned in the response. As a result, clients may need to retrieve the modified calendar object resource (and ETag) as a basis for further changes, rather than use the calendar object resource it had sent with the PUT request. 


\section{Calendaring Access Control}

\subsection{Calendaring Privilege}

CalDAV servers MUST support and adhere to the requirements of WebDAV ACL [RFC3744]. WebDAV ACL provides a framework for an extensible set of privileges that can be applied to WebDAV collections and ordinary resources. CalDAV servers MUST also support the calendaring privilege defined in this section.

\subsubsection{CALDAV:read-free-busy Privilege}

Calendar users often wish to allow other users to see their busy time information, without viewing the other details of the calendar components (e.g., location, summary, attendees). This allows a significant amount of privacy while still allowing other users to schedule meetings at times when the user is likely to be free.

The CALDAV:read-free-busy privilege controls which calendar collections, regular collections, and calendar object resources are examined when a CALDAV:free-busy-query REPORT request is processed (see Section 7.10). This privilege can be granted on calendar collections, regular collections, or calendar object resources. Servers MUST support this privilege on all calendar collections, regular collections, and calendar object resources.

$$
\text { <!ELEMENT read-free-busy EMPTY> }
$$

The CALDAV:read-free-busy privilege MUST be aggregated in the DAV:read privilege. Servers MUST allow the CALDAV:read-free-busy to be granted without the DAV:read privilege being granted.

Clients should note that when only the CALDAV:read-free-busy privilege has been granted on a resource, access to GET, HEAD, OPTIONS, and PROPFIND on the resource is not implied (those operations are governed by the DAV:read privilege).

\subsection{Additional Principal Property}

This section defines an additional property for WebDAV principal resources, as defined in [RFC3744].

\subsubsection{CALDAV:calendar-home-set Property}

Name:

Namespace:

Purpose:

Conformance:

Description:

Definition: calendar-home-set

urn:ietf:params:xml:ns:caldav

Identifies the URL of any WebDAV collections that contain calendar collections owned by the associated principal resource.

This property SHOULD be defined on a principal resource. If defined, it MAY be protected and SHOULD NOT be returned by a PROPFIND DAV:allprop request (as defined in Section 12.14.1 of [RFC2518]).

The CALDAV:calendar-home-set property is meant to allow users to easily find the calendar collections owned by the principal. Typically, users will group all the calendar collections that they own under a common collection. This property specifies the URL of collections that are either calendar collections or ordinary collections that have child or descendant calendar collections owned by the principal.

$$
<\text { !ELEMENT calendar-home-set (DAV:href*) > }
$$


Example:

$<$ C: calendar-home-set xmlns:D="DAV:"

xmlns: $\mathrm{C}=$ "urn: ietf $:$ params: $\mathrm{xml}: \mathrm{ns}: \mathrm{caldav">}$

<D: href > http://cal.example.com/home/bernard/

calendars/ $</ D:$ href $>$

$</$ C:calendar-home-set $>$ 


\section{Calendaring Reports}

This section defines the reports that CaIDAV servers MUST support on calendar collections and calendar object resources.

CalDAV servers MUST advertise support for these reports on all calendar collections and calendar object resources with the DAV:supported-report-set property, defined in Section 3.1.5 of [RFC3253]. CalDAV servers MAY also advertise support for these reports on ordinary collections.

Some of these reports allow calendar data (from possibly multiple resources) to be returned.

\subsection{REPORT Method}

The REPORT method (defined in Section 3.6 of [RFC3253]) provides an extensible mechanism for obtaining information about one or more resources. Unlike the PROPFIND method, which returns the value of one or more named properties, the REPORT method can involve more complex processing. REPORT is valuable in cases where the server has access to all of the information needed to perform the complex request (such as a query), and where it would require multiple requests for the client to retrieve the information needed to perform the same request.

CalDAV servers MUST support the DAV:expand-property REPORT defined in Section 3.8 of [RFC3253].

\subsection{Ordinary Collections}

Servers MAY support the reports defined in this document on ordinary collections (collections that are not calendar collections), in addition to calendar collections or calendar object resources. In computing responses to the reports on ordinary collections, servers MUST only consider calendar object resources contained in calendar collections that are targeted by the REPORT request, based on the value of the Depth request header.

\subsection{Date and Floating Time}

iCalendar provides a way to specify DATE and DATE-TIME values that are not bound to any time zone in particular, hereafter called "floating date" and "floating time", respectively. These values are used to represent the same day, hour, minute, and second value, regardless of which time zone is being observed. For instance, the DATE value "20051111", represents November 11, 2005 in no specific time zone, while the DATE-TIME value "20051111T111100" represents November 11, 2005, at 11:11 A.M. in no specific time zone.

CalDAV servers may need to convert "floating date" and "floating time" values in date with UTC time values in the processing of calendaring REPORT requests.

For the CALDAV:calendar-query REPORT, CalDAV servers MUST rely on the value of the CALDAV:timezone XML element, if specified as part of the request body, to perform the proper conversion of "floating date" and "floating time" values to date with UTC time values. If the CALDAV:timezone XML element is not specified in the request body, CalDAV servers MUST rely on the value of the CALDAV:calendar-timezone property, if defined, or else the CalDAV servers MAY rely on the time zone of their choice.

For the CALDAV:free-busy-query REPORT, CalDAV servers MUST rely on the value of the CALDAV:calendar-timezone property, if defined, to compute the proper FREEBUSY time period value as date with UTC time for calendar components scheduled with "floating date" or "floating time". If the CALDAV:calendar-timezone property is not defined, CalDAV servers MAY rely on the time zone of their choice.

\subsection{Time Range Filtering}

Some of the reports defined in this section can include a time range filter that is used to restrict the set of calendar object resources returned to just those that overlap the specified time range. The time range filter can 
be applied to a calendar component as a whole, or to specific calendar component properties with DATE or DATE-TIME value types.

To determine whether a calendar object resource matches the time range filter element, the start and end times for the targeted component or property are determined and then compared to the requested time range. If there is an overlap with the requested time range, then the calendar object resource matches the filter element. The rules defined in [RFC2445] for determining the actual start and end times of calendar components MUST be used, and these are fully enumerated in Section 9.9 of this document.

When such time range filtering is used, special consideration must be given to recurring calendar components, such as VEVENT and VTODO. The server MUST expand recurring components to determine whether any recurrence instances overlap the specified time range. If one or more recurrence instances overlap the time range, then the calendar object resource matches the filter element.

\subsection{Searching Text: Collations}

Some of the reports defined in this section do text matches of character strings provided by the client and are compared to stored calendar data. Since iCalendar data is, by default, encoded in the UTF-8 charset and may include characters outside the US-ASCII charset range in some property and parameter values, there is a need to ensure that text matching follows well-defined rules.

To deal with this, this specification makes use of the IANA Collation Registry defined in [RFC4790] to specify collations that may be used to carry out the text comparison operations with a well-defined rule.

The comparisons used in CalDAV are all "substring" matches, as per [RFC4790], Section 4.2. Collations supported by the server MUST support "substring" match operations.

CalDAV servers are REQUIRED to support the "i;ascii-casemap" and "i;octet" collations, as described in [RFC4790], and MAY support other collations.

Servers MUST advertise the set of collations that they support via the CALDAV:supported-collation-set property defined on any resource that supports reports that use collations.

Clients MUST only use collations from the list advertised by the server.

In the absence of a collation explicitly specified by the client, or if the client specifies the "default" collation identifier (as defined in [RFC4790], Section 3.1), the server MUST default to using "i;ascii-casemap" as the collation.

Wildcards (as defined in [RFC4790], Section 3.2) MUST NOT be used in the collation identifier.

If the client chooses a collation not supported by the server, the server MUST respond with a CALDAV:supported-collation precondition error response.

\subsubsection{CALDAV:supported-collation-set Property}

Name:

Namespace:

Purpose:

Conformance:

Description: supported-collation-set

urn:ietf:params:xml:ns:caldav

Identifies the set of collations supported by the server for text matching operations.

This property MUST be defined on any resource that supports a report that does text matching. If defined, it MUST be protected and SHOULD NOT be returned by a PROPFIND DAV:allprop request (as defined in Section 12.14.1 of [RFC2518]).

The CALDAV:supported-collation-set property contains zero or more CALDAV:supported-collation elements, which specify the collection identifiers of the collations supported by the server. 
Definition:

$$
\begin{aligned}
& \text { <!ELEMENT supported-collation-set (supported- } \\
& \text { Collation*)> } \\
& <\text { !ELEMENT supported-collation (\#PCDATA) > }
\end{aligned}
$$

Example:

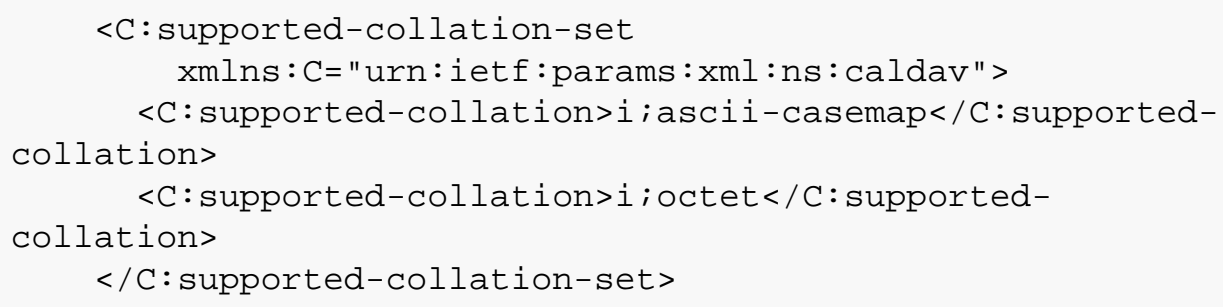

\subsection{Partial Retrieval}

Some calendaring reports defined in this document allow partial retrieval of calendar object resources. A CalDAV client can specify what information to return in the body of a calendaring REPORT request.

A CalDAV client can request particular WebDAV property values, all WebDAV property values, or a list of the names of the resource's WebDAV properties. A CalDAV client can also request calendar data to be returned and specify whether all calendar components and properties should be returned, or only particular ones. See CALDAV:calendar-data in Section 9.6.

By default, the returned calendar data will include the component that defines the recurrence set, referred to as the "master component", as well as the components that define exceptions to the recurrence set, referred to as the "overridden components".

A CalDAV client that is only interested in the recurrence instances that overlap a specified time range can request to receive only the "master component", along with the "overridden components" that impact the specified time range, and thus, limit the data returned by the server (see CALDAV:limit-recurrence-set in Section 9.6.6). An overridden component impacts a time range if its current start and end times overlap the time range, or if the original start and end times -- the ones that would have been used if the instance were not overridden -- overlap the time range, or if it affects other instances that overlap the time range.

A CalDAV client with no support for recurrence properties (i.e., EXDATE, EXRULE, RDATE, and RRULE) and possibly VTIMEZONE components, or a client unwilling to perform recurrence expansion because of limited processing capability, can request to receive only the recurrence instances that overlap a specified time range as separate calendar components that each define exactly one recurrence instance (see CALDAV:expand in Section 9.6.5.)

Finally, in the case of VFREEBUSY components, a CalDAV client can request to receive only the FREEBUSY property values that overlap a specified time range (see CALDAV:limit-freebusy-set in Section 9.6.7.)

\subsection{Non-Standard Components, Properties, and Parameters}

Servers MUST support the use of non-standard component, property, or parameter names in the CALDAV:calendar-data XML element in calendaring REPORT requests to allow clients to request that nonstandard components, properties, and parameters be returned in the calendar data provided in the response.

Servers MAY support the use of non-standard component, property, or parameter names in the CALDAV:comp-filter, CALDAV:prop-filter, and CALDAV:param-filter XML elements specified in the CALDAV:filter XML element of calendaring REPORT requests.

Servers MUST fail with the CALDAV:supported-filter precondition if a calendaring REPORT request uses a CALDAV:comp-filter, CALDAV:prop-filter, or CALDAV:param-filter XML element that makes reference to a non-standard component, property, or parameter name on which the server does not support queries. 


\subsection{CALDAV:calendar-query REPORT}

The CALDAV:calendar-query REPORT performs a search for all calendar object resources that match a specified filter. The response of this report will contain all the WebDAV properties and calendar object resource data specified in the request. In the case of the CALDAV:calendar-data XML element, one can explicitly specify the calendar components and properties that should be returned in the calendar object resource data that matches the filter.

The format of this report is modeled on the PROPFIND method. The request and response bodies of the CALDAV:calendar-query REPORT use XML elements that are also used by PROPFIND. In particular, the request can include XML elements to request WebDAV properties to be returned. When that occurs, the response should follow the same behavior as PROPFIND with respect to the DAV:multistatus response elements used to return specific property results. For instance, a request to retrieve the value of a property that does not exist is an error and MUST be noted with a response XML element that contains a 404 (Not Found) status value.

Support for the CALDAV:calendar-query REPORT is REQUIRED.

\section{Marshalling:}

The request body MUST be a CALDAV:calendar-query XML element, as defined in Section 9.5. The request MAY include a Depth header. If no Depth header is included, Depth:0 is assumed.

The response body for a successful request MUST be a DAV:multistatus XML element (i.e., the response uses the same format as the response for PROPFIND). In the case where there are no response elements, the returned DAV:multistatus XML element is empty.

The response body for a successful CALDAV:calendar-query REPORT request MUST contain a DAV:response element for each iCalendar object that matched the search filter. Calendar data is being returned in the CALDAV:calendar-data XML element inside the DAV:propstat XML element.

\section{Preconditions:}

(CALDAV:supported-calendar-data): The attributes "content-type" and "version" of the CALDAV:calendar-data XML element (see Section 9.6) specify a media type supported by the server for calendar object resources.

(CALDAV:valid-filter): The CALDAV:filter XML element (see Section 9.7) specified in the REPORT request MUST be valid. For instance, a CALDAV:filter cannot nest a $\langle\mathrm{C}:$ comp name="VEVENT" $\rangle$ element in a $\langle\mathrm{C}$ :comp name="VTODO" $>$ element, and a CALDAV:filter cannot nest a $<\mathrm{C}$ :time-range start="..." end="..."> element in a $<\mathrm{C}$ :prop name="SUMMARY"> element.

(CALDAV:supported-filter): The CALDAV:comp-filter (see Section 9.7.1), CALDAV:prop-filter (see Section 9.7.2), and CALDAV:param-filter (see Section 9.7.3) XML elements used in the CALDAV:filter XML element (see Section 9.7) in the REPORT request only make reference to components, properties, and parameters for which queries are supported by the server, i.e., if the CALDAV:filter element attempts to reference an unsupported component, property, or parameter, this precondition is violated. Servers SHOULD report the CALDAV:comp-filter, CALDAV:prop-filter, or CALDAV:param-filter for which it does not provide support.

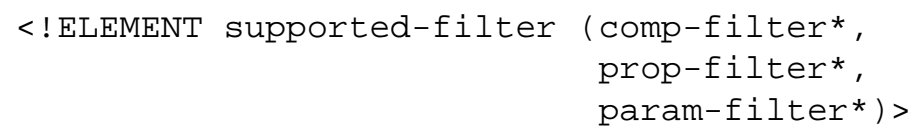

(CALDAV:valid-calendar-data): The time zone specified in the REPORT request MUST be a valid iCalendar object containing a single valid VTIMEZONE component.

(CALDAV:min-date-time): Any XML element specifying a range of time MUST have its start or end DATE or DATE-TIME values greater than or equal to the value of the CALDAV:min-date-time property value (Section 5.2.6) on the calendar collections being targeted by the REPORT request; 
(CALDAV:max-date-time): Any XML element specifying a range of time MUST have its start or end DATE or DATE-TIME values less than or equal to the value of the CALDAV:max-date-time property value (Section 5.2.7) on the calendar collections being targeted by the REPORT request;

(CALDAV:supported-collation): Any XML attribute specifying a collation MUST specify a collation supported by the server as described in Section 7.5.

\section{Postconditions:}

(DAV:number-of-matches-within-limits): The number of matching calendar object resources must fall within server-specific, predefined limits. For example, this condition might be triggered if a search specification would cause the return of an extremely large number of responses.

\subsubsection{Example: Partial Retrieval of Events by Time Range}

In this example, the client requests the server to return specific components and properties of the VEVENT components that overlap the time range from January 4, 2006, at 00:00:00 A.M. UTC to January 5, 2006, at 00:00:00 A.M. UTC. In addition, the DAV:getetag property is also requested and returned as part of the response. Note that the first calendar object returned is a recurring event whose first instance lies outside the requested time range, but whose third instance does overlap the time range. Note that due to the CALDAV:calendar-data element restrictions, the DTSTAMP property in VEVENT components has not been returned, and the only property returned in the VCALENDAR object is VERSION.

See Appendix B for the calendar data being targeted by this example. 


\section{$>$ Request $<<$}

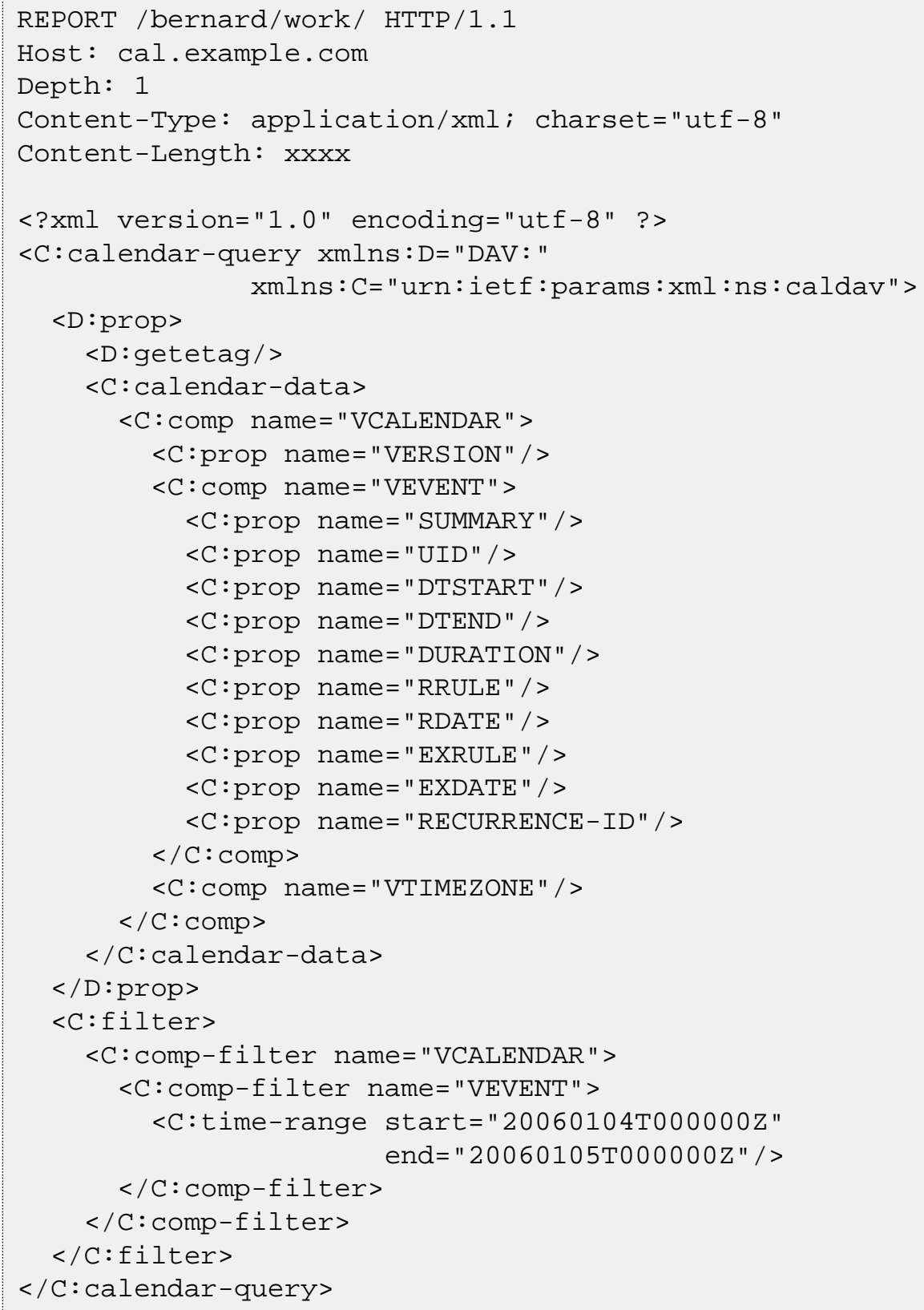


$>>$ Response $<<$

HTTP/1.1 207 Multi-Status

Date: Sat, 11 Nov 2006 09:32:12 GMT

Content-Type: application/xml; charset="utf-8"

Content-Length: $\mathrm{xxxx}$

<?xml version="1.0" encoding="utf-8" ?>

$<$ D:multistatus xmlns:D="DAV:"

xmlns: $\mathrm{C}=$ "urn: ietf $:$ params: $\mathrm{xml}: \mathrm{ns}: \mathrm{caldav">}$

$<\mathrm{D}:$ response>

$<\mathrm{D}:$ href $>$ http: / / cal . example.com/bernard/work/abcd2.ics</D: href $>$

$<$ D:propstat $>$

$<\mathrm{D}:$ prop $>$

$<$ D : getetag $>$ "fffff-abcd2" </D : getetag $>$

$<$ C:calendar-data>BEGIN : VCALENDAR

VERSION : 2.0

BEGIN : VTIMEZONE

LAST-MODIFIED : 20040110 T032845Z

TZID : US/Eastern

BEGIN : DAYLIGHT

DTSTART:20000404T020000

RRULE : $F R E Q=Y E A R L Y ; B Y D A Y=1 S U$; $B Y M O N T H=4$

TZNAME : EDT

TZOFFSETFROM: -0500

TZOFFSETTO: -0400

END : DAYLIGHT

BEGIN : STANDARD

DTSTART:20001026T020000

RRULE : $F R E Q=Y E A R L Y ; B Y D A Y=-1 S U$; $B Y M O N T H=10$

TZNAME :EST

TZOFFSETFROM: -0400

TZOFFSETTO: -0500

END : STANDARD

END : VTIMEZONE

BEGIN : VEVENT

DTSTART; TZID=US/Eastern :20060102T120000

DURATION : PT1H

RRULE : FREQ=DAILY; $C O U N T=5$

SUMMARY:Event \#2

UID : 0095 9BC664CA650E933C892C@example.com

END : VEVENT

BEGIN : VEVENT

DTSTART; TZID=US /Eastern:20060104T140000

DURATION : PT1H

RECURRENCE-ID; TZID=US / Eastern : $20060104 \mathrm{~T} 120000$

SUMMARY:Event \#2 bis

UID : 00959BC664CA650E933C892C@example.com

END : VEVENT

BEGIN : VEVENT

DTSTART; TZID=US/Eastern:20060106T140000

DURATION : PT1H

RECURRENCE-ID; TZID=US / Eastern : $20060106 \mathrm{~T} 120000$

SUMMARY:Event \#2 bis bis

UID : 0095 9BC664CA650E933C892C dexample.com

END : VEVENT

END : VCALENDAR

</C:calendar-data $>$

Daboo, et al. </D : prop $>$

Standards Track

[Page 31] $<$ D: status $>$ HTTP/1.1 200 OK</D:status $>$ 


\subsubsection{Example: Partial Retrieval of Recurring Events}

In this example, the client requests the server to return VEVENT components that overlap the time range from January 3, 2006, at 00:00:00 A.M. UTC to January 5, 2006, at 00:00:00 A.M. UTC. Use of the CALDAV:limit-recurrence-set element causes the server to only return overridden recurrence components that overlap the time range specified in that element or that affect other instances that overlap the time range (e.g., in the case of a THISANDFUTURE behavior). In this example, the first overridden component in the matching resource is returned, but the second one is not.

See Appendix B for the calendar data being targeted by this example.

$>$ Request $<<$

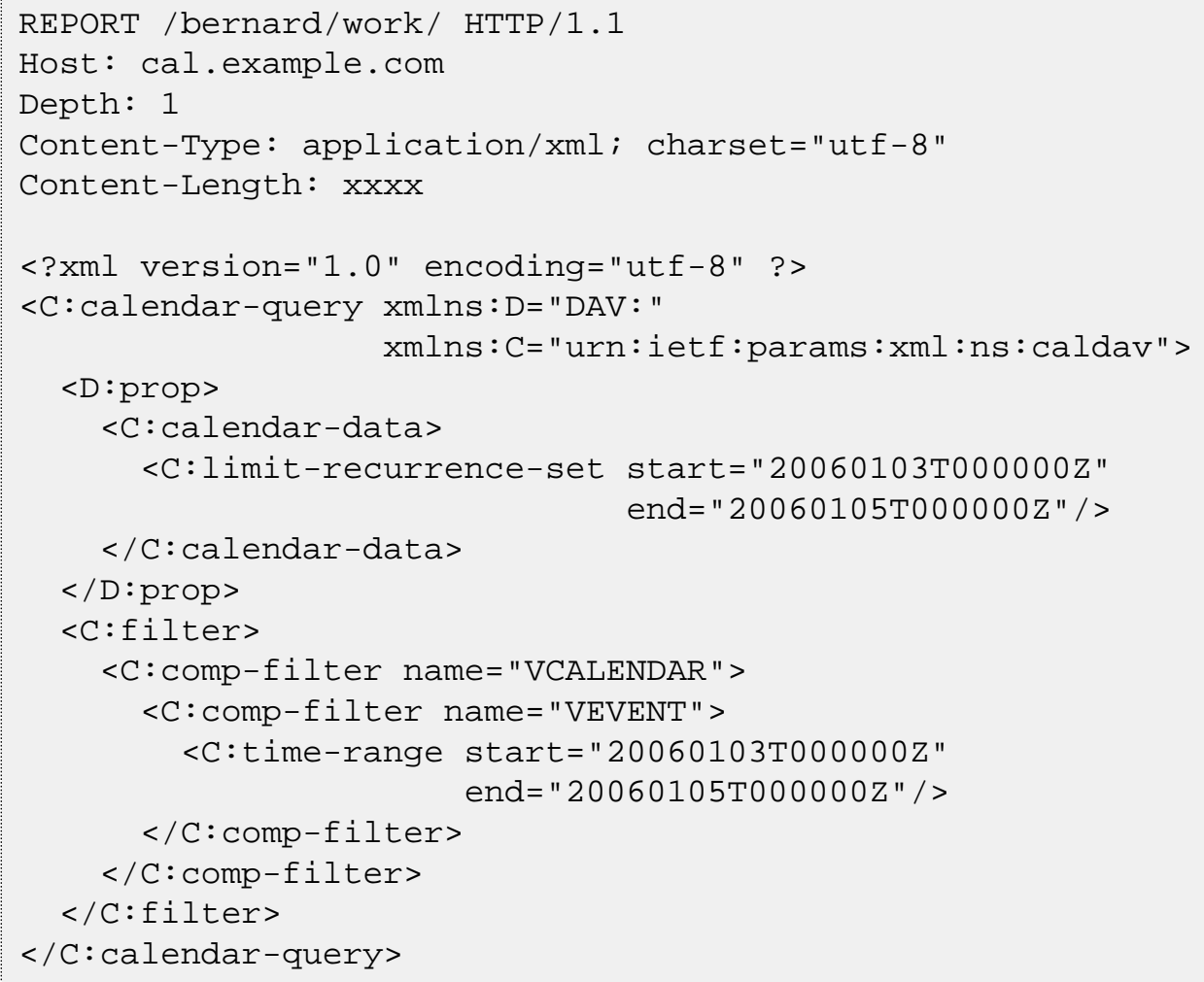




\section{$>$ Response $<<$}

HTTP/1.1 207 Multi-Status

Date: Sat, 11 Nov 2006 09:32:12 GMT

Content-Type: application/xml; charset="utf-8"

Content-Length: $\mathrm{xxxx}$

<?xml version="1.0" encoding="utf-8" ?>

$<$ : multistatus xmlns:D="DAV:"

xmlns:C="urn: ietf $:$ params: $x m l: n s:$ caldav">

$<\mathrm{D}:$ response>

$<$ D : href>http: / / cal . example.com/bernard/work/abcd2.ics</D: href>

$<\mathrm{D}:$ propstat $>$

$<\mathrm{D}:$ prop $>$

$<$ D : getetag $>$ "fffff-abcd2" </D : getetag $>$

$<$ C:calendar-data>BEGIN : VCALENDAR

VERSION : 2.0

PRODID:-//Example Corp.//CalDAV Client//EN

BEGIN : VTIMEZONE

LAST-MODIFIED : 20040110 T032845Z

TZID:US/Eastern

BEGIN : DAYLIGHT

DTSTART : $20000404 T 020000$

RRULE : $F R E Q=Y E A R L Y$; $B Y D A Y=1 S U$; $B Y M O N T H=4$

TZNAME : EDT

TZOFFSETFROM: -0500

TZOFFSETTO: -0400

END :DAYLIGHT

BEGIN : STANDARD

DTSTART : $20001026 T 020000$

RRULE : $F R E Q=Y E A R L Y ; B Y D A Y=-1 S U$; $B Y M O N T H=10$

TZNAME :EST

TZOFFSETFROM: -0400

TZOFFSETTO:-0500

END : STANDARD

END : VTIMEZONE

BEGIN : VEVENT

DTSTAMP :20060206T001121Z

DTSTART; TZID=US/Eastern:20060102T120000

DURATION : PT1H

RRULE : $F R E Q=D A I L Y ; C O U N T=5$

SUMMARY:Event \#2

UID : 0095 9BC6 64CA650E933C892C@example.com

END : VEVENT

BEGIN : VEVENT

DTSTAMP : $20060206 \mathrm{~T} 001121 \mathrm{Z}$

DTSTART; TZID=US/Eastern:20060104T140000

DURATION :PT1H

RECURRENCE-ID; TZID=US / Eastern : $20060104 \mathrm{~T} 120000$

SUMMARY:Event \#2 bis

UID:00959BC664CA650E933C892C@example.com

END : VEVENT

END : VCALENDAR

$</ C$ : calendar-data $>$

$</ \mathrm{D}:$ prop $>$

$<$ D:status $>$ HTTP/1.1 200 OK</D:status $>$

$</ D$ :propstat $>$

$</ \mathrm{D}$ : response $>$

Daboo, etpal.response>

Standards Track

[Page 33]

<D: href>http://cal.example.com/bernard/work/abcd3.ics</D: href > 


\subsubsection{Example: Expanded Retrieval of Recurring Events}

In this example, the client requests the server to return VEVENT components that overlap the time range from January 2, 2006, at 00:00:00 A.M. UTC to January 5, 2006, at 00:00:00 A.M. UTC and to return recurring calendar components expanded into individual recurrence instance calendar components. Use of the CALDAV:expand element causes the server to only return overridden recurrence instances that overlap the time range specified in that element.

See Appendix B for the calendar data being targeted by this example.

$>$ Request $<<$

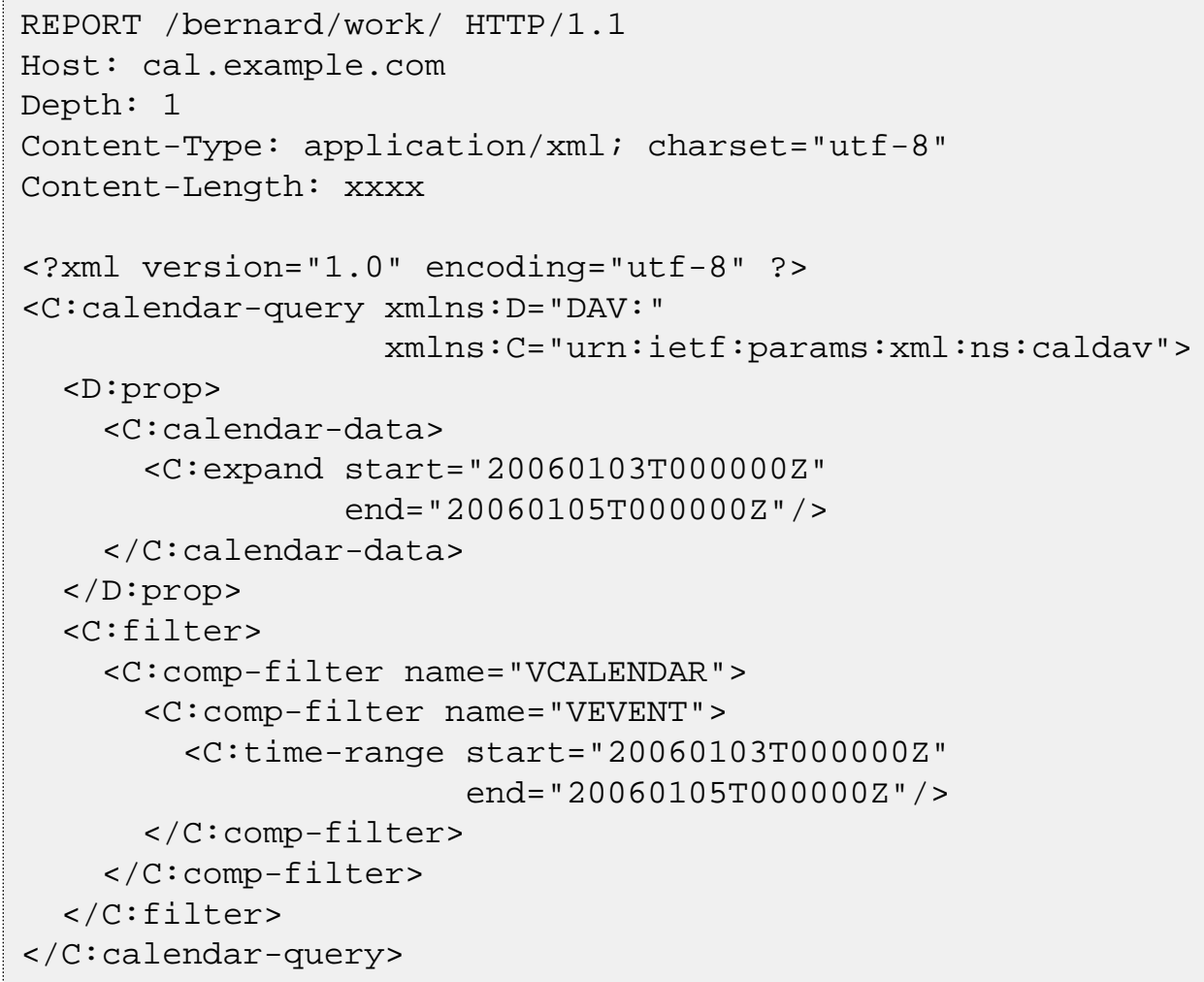




\section{$>$ Response $<<$}

HTTP/1.1 207 Multi-Status

Date: Sat, 11 Nov 2006 09:32:12 GMT

Content-Type: application/xml; charset="utf-8"

Content-Length: $\mathrm{xxxx}$

<?xml version="1.0" encoding="utf-8" ?>

$<$ : multistatus xmlns:D="DAV:"

xmlns:C="urn: ietf $:$ params: $x m l: n s:$ caldav">

$<\mathrm{D}:$ response>

$<$ D : href>http: / / cal . example.com/bernard/work/abcd2.ics</D: href>

$<\mathrm{D}:$ propstat $>$

$<\mathrm{D}:$ prop $>$

$<$ D : getetag $>$ "fffff-abcd2" </D : getetag $>$

$<$ C:calendar-data>BEGIN :VCALENDAR

VERSION : 2.0

PRODID:-//Example Corp.//CalDAV Client//EN

BEGIN : VEVENT

DTSTAMP : $20060206 \mathrm{~T} 001121 \mathrm{Z}$

DTSTART : $20060103 T 170000$

DURATION :PT $1 \mathrm{H}$

RECURRENCE-ID:20060103T170000

SUMMARY : Event \#2

UID : 00959 BC 664 CA 650E933C892C@example.com

END : VEVENT

BEGIN : VEVENT

DTSTAMP : $20060206 \mathrm{~T} 001121 \mathrm{Z}$

DTSTART : $20060104 \mathrm{~T} 190000$

DURATION : PT1H

RECURRENCE-ID: $20060104 \mathrm{~T} 170000$

SUMMARY:Event \#2 bis

UID : 0095 9BC664CA650E933C892C@example.com

END : VEVENT

END : VCALENDAR

$</$ C:calendar-data $>$

$</ \mathrm{D}:$ prop $>$

$<$ D:status $>$ HTTP/1.1 200 OK</D:status $>$

$</ \mathrm{D}:$ propstat $>$

$</ \mathrm{D}:$ response $>$

$<\mathrm{D}:$ response $>$

$<$ D : href>http: / / cal . example.com/bernard/work/abcd3.ics</D:href>

$<\mathrm{D}$ : propstat $>$

$<\mathrm{D}:$ prop $>$

$<$ D: getetag>"fffff-abcd3"</D: getetag>

$<C$ : calendar-data>BEGIN : VCALENDAR

VERSION $: 2.0$

PRODID:-//Example Corp.//CalDAV Client//EN

BEGIN : VEVENT

ATTENDEE; PARTSTAT=ACCEPTED; ROLE=CHAIR : mailto : cyrusdexample. com

ATTENDEE; PARTSTAT=NEEDS-ACTION : mailto:lisa@example.com

DTSTAMP : $20060206 \mathrm{~T} 001220 \mathrm{Z}$

DTSTART : $20060104 \mathrm{~T} 150000$

DURATION :PT1H

LAST-MODIF IED : 20060206 T001330Z

ORGANIZER:mailto: cyrus@example.com

SEQUENCE : 1

STATUS : TENTATIVE

DaboguAtA

Standards Track

[Page 35]

UID:DC6C50A017428C5216A2F1CD@example.com 


\subsubsection{Example: Partial Retrieval of Stored Free Busy Components}

In this example, the client requests the server to return the VFREEBUSY components that have free busy information that overlap the time range from January 2, 2006, at 00:00:00 A.M. UTC (inclusively) to January 3, 2006, at 00:00:00 A.M. UTC (exclusively). Use of the CALDAV:limit-freebusy-set element causes the server to only return the FREEBUSY property values that overlap the time range specified in that element. Note that this is not an example of discovering when the calendar owner is busy.

See Appendix B for the calendar data being targeted by this example.

$>$ Request $<<$

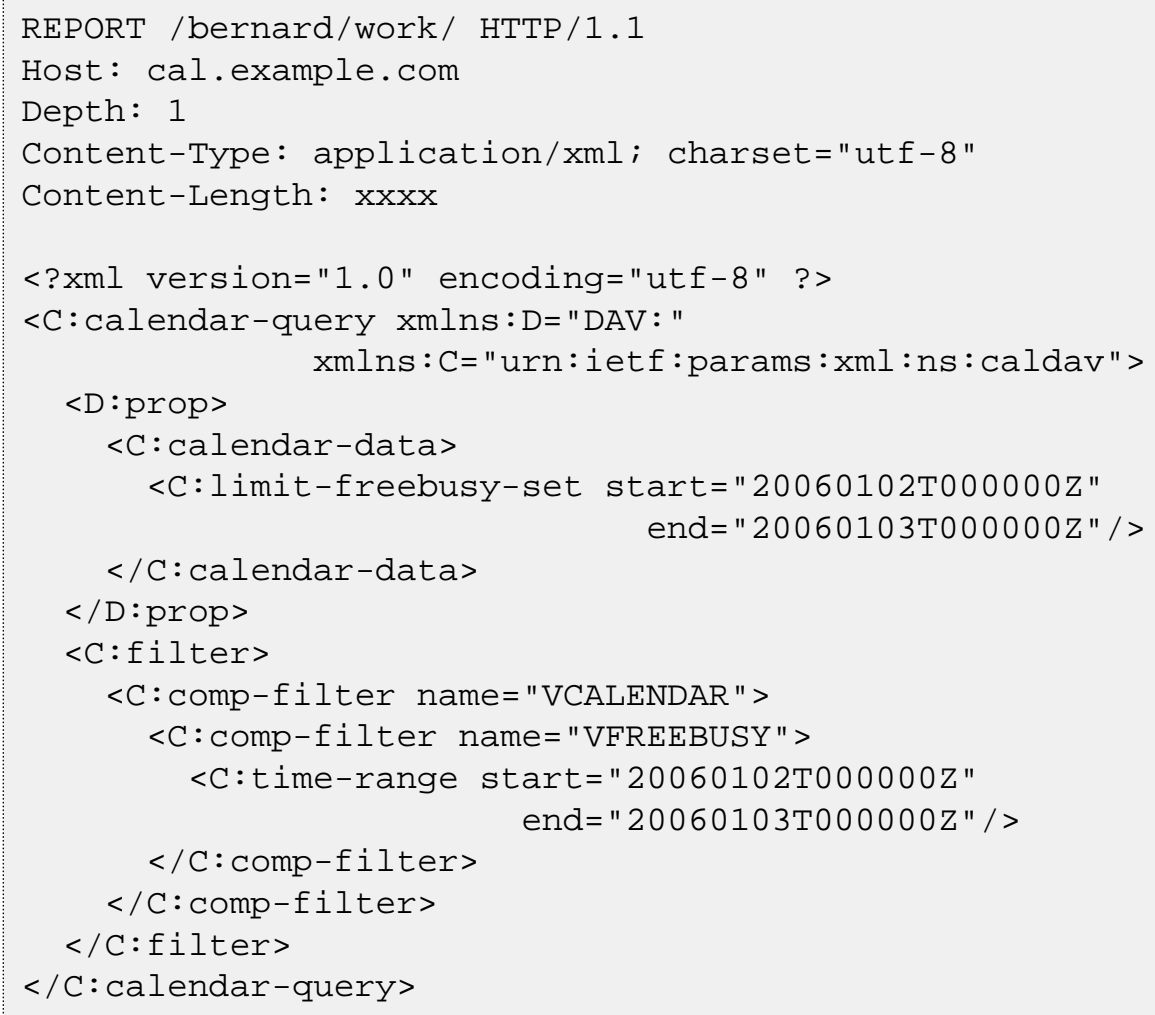




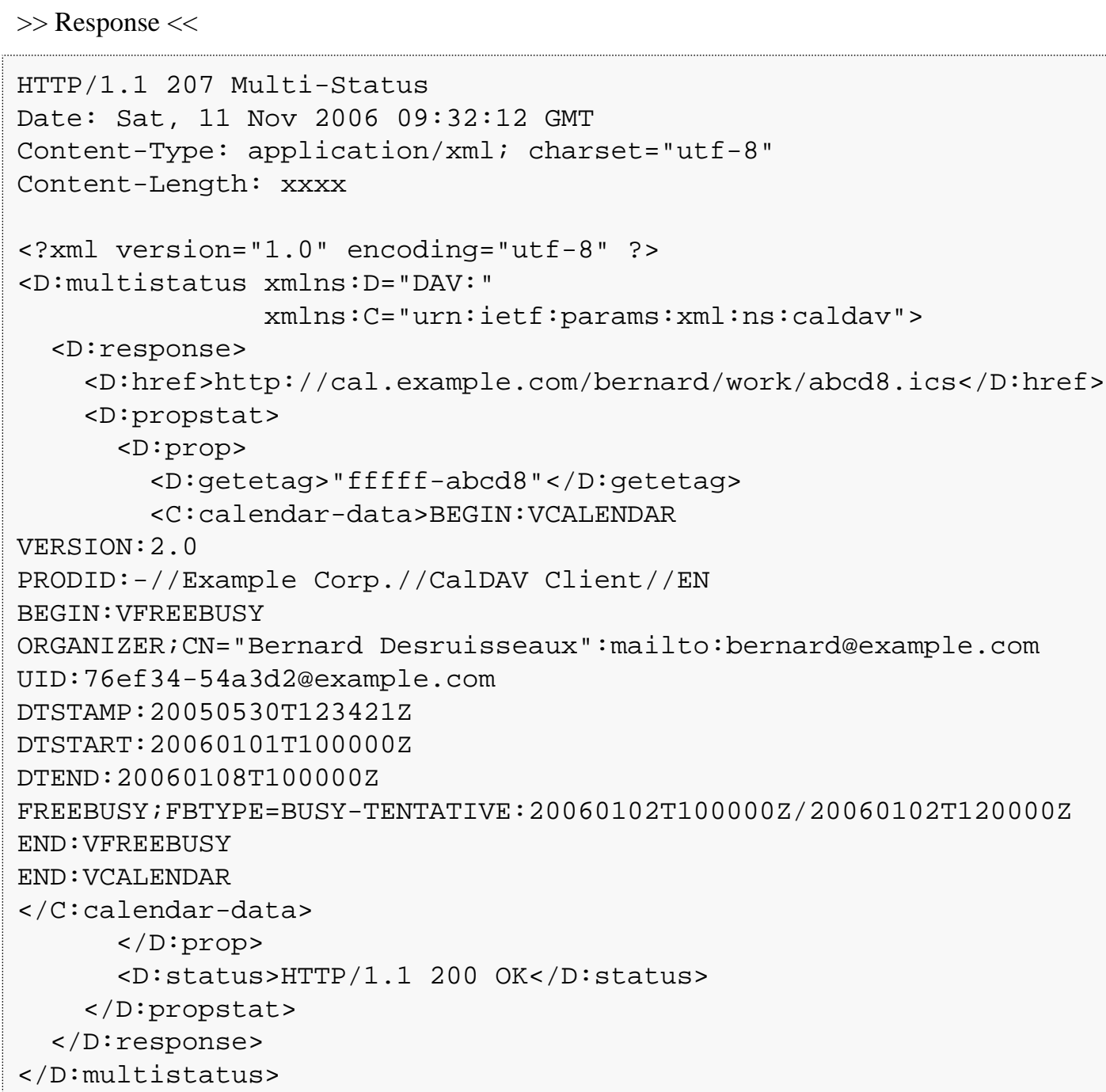

\subsubsection{Example: Retrieval of To-Dos by Alarm Time Range}

In this example, the client requests the server to return the VTODO components that have an alarm trigger scheduled in the specified time range.

See Appendix B for the calendar data being targeted by this example. 


\section{$>$ Request $<<$}

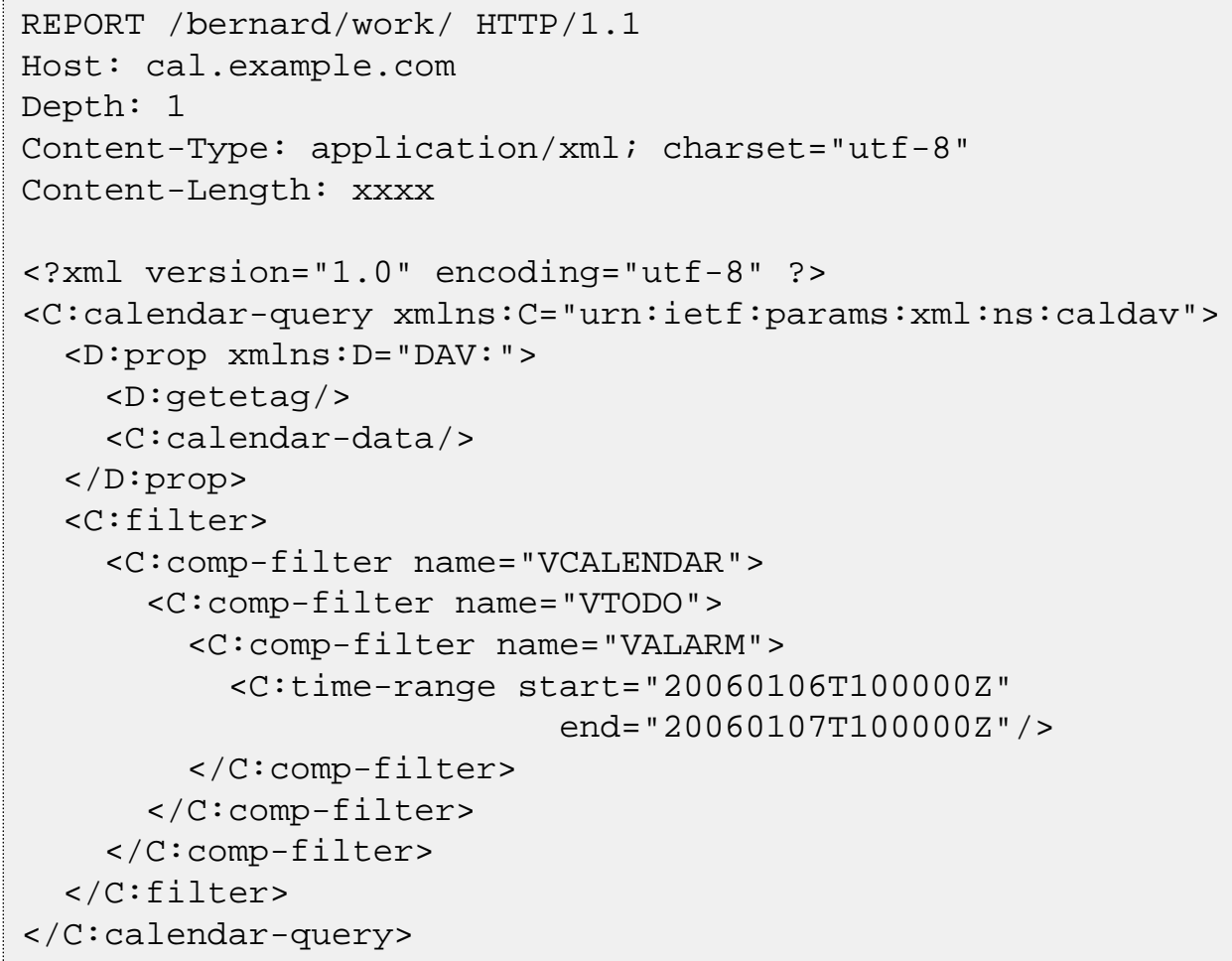




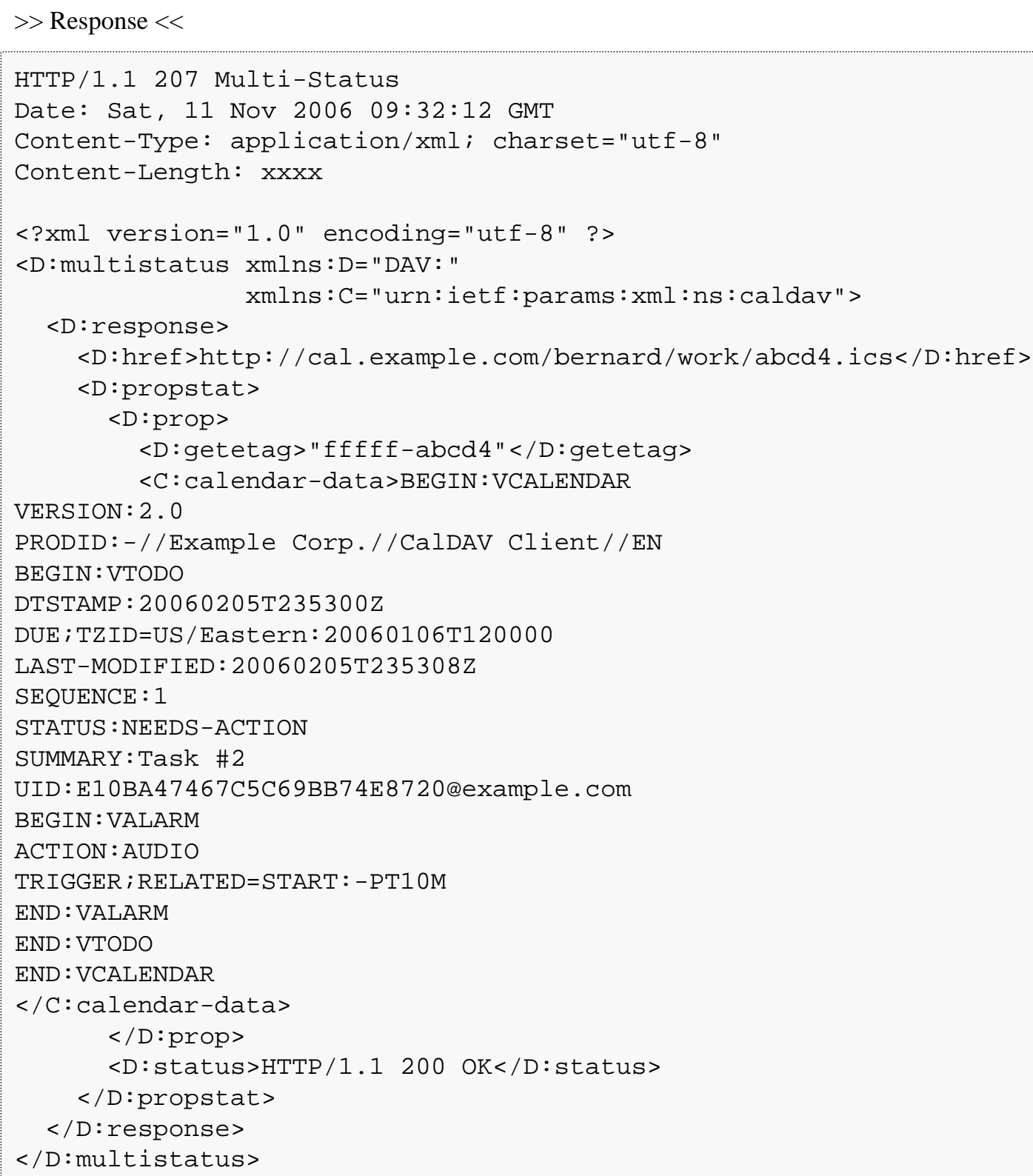

\subsubsection{Example: Retrieval of Event by UID}

In this example, the client requests the server to return the VEVENT component that has the UID property set to "DC6C50A017428C5216A2F1CD@example.com".

See Appendix B for the calendar data being targeted by this example. 


\section{> Request $<<$}

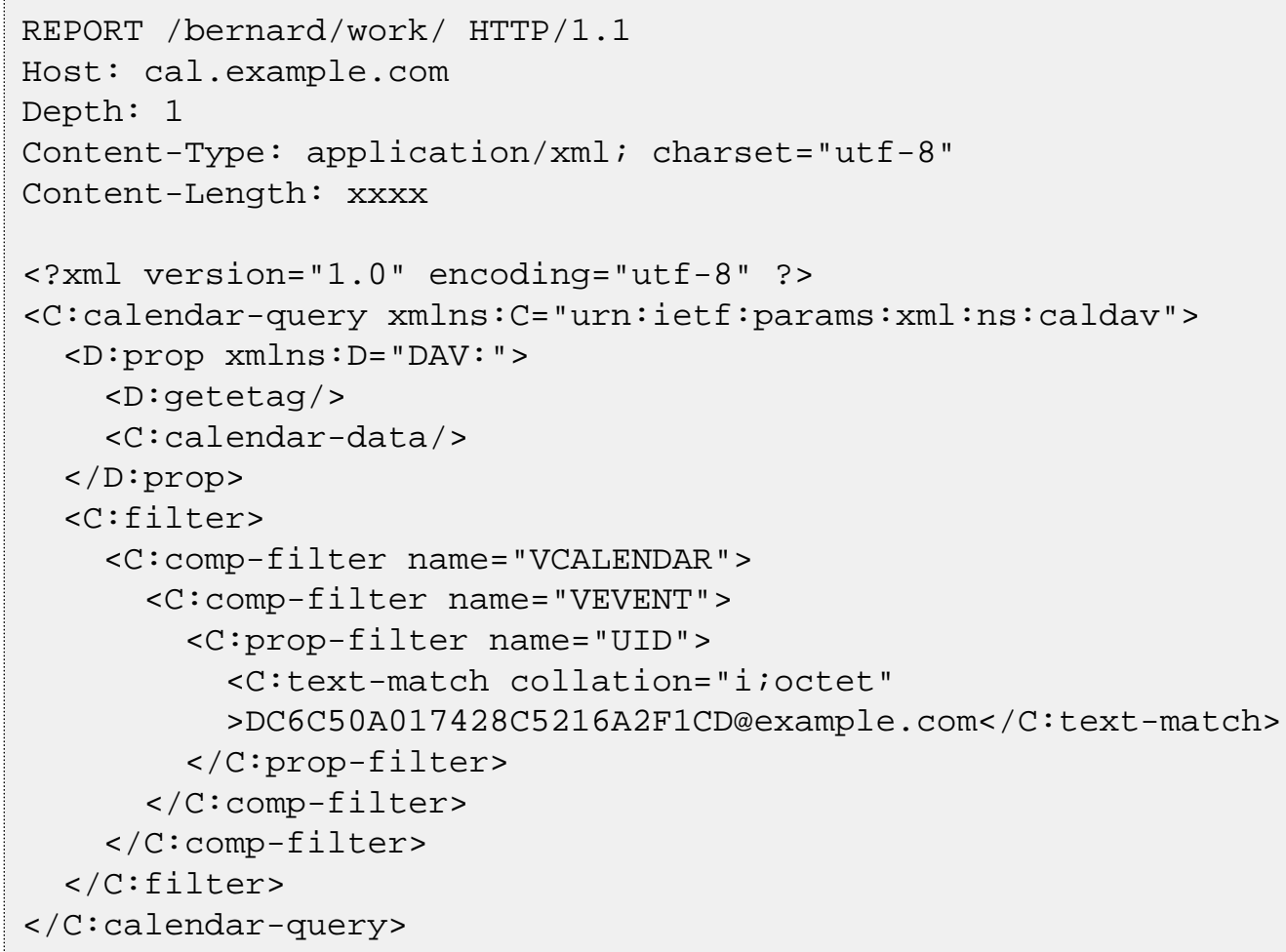




\section{$>$ Response $<<$}

HTTP/1.1 207 Multi-Status

Date: Sat, 11 Nov 2006 09:32:12 GMT

Content-Type: application/xml; charset="utf-8"

Content-Length: $\mathrm{xxxx}$

<?xml version="1.0" encoding="utf-8" ?>

$<$ D:multistatus xmlns:D="DAV:"

xmlns:C="urn: ietf $:$ params: $x m l: n s:$ caldav">

$<\mathrm{D}:$ response>

$<$ D: href $>$ http: //cal . example.com/bernard/work/abcd3.ics</D:href $>$

$<$ D:propstat $>$

$<\mathrm{D}:$ prop $>$

$<$ D : getetag $>$ "fffff-abcd3"</D: getetag $>$

$<$ C:calendar-data>BEGIN : VCALENDAR

VERSION : 2.0

PRODID:-//Example Corp.//CalDAV Client//EN

BEGIN : VTIMEZONE

LAST-MODIFIED : 20040110 T032845Z

TZID:US/Eastern

BEGIN : DAYLIGHT

DTSTART : $20000404 T 020000$

RRULE : $F R E Q=Y E A R L Y$; $B Y D A Y=1 S U$; $B Y M O N T H=4$

TZNAME : EDT

TZOFFSETFROM: -0500

TZOFFSETTO: -0400

END : DAYLIGHT

BEGIN : STANDARD

DTSTART : $20001026 T 020000$

RRULE : $F R E Q=Y E A R L Y ; B Y D A Y=-1 S U$; $B Y M O N T H=10$

TZNAME : EST

TZOFFSETFROM: -0400

TZOFFSETTO:-0500

END : STANDARD

END : VTIMEZONE

BEGIN : VEVENT

ATTENDEE; PARTSTAT=ACCEPTED; ROLE=CHAIR : mailto : cyrusdexample. com

ATTENDEE; PARTSTAT=NEEDS-ACTION : mailto:lisadexample.com

DTSTAMP : $20060206 \mathrm{~T} 001220 \mathrm{Z}$

DTSTART; TZID=US/Eastern:20060104T100000

DURATION : PT $1 \mathrm{H}$

LAST-MODIFIED : 20060206 T001330Z

ORGANIZER:mailto: cyrus@example.com

SEQUENCE : 1

STATUS : TENTATIVE

SUMMARY:Event \#3

UID : DC6C50A017428C5216A2F1CD@example.com

$\mathrm{X}-\mathrm{ABC}-\mathrm{GUID}: \mathrm{E} 1 \mathrm{CX} 5 \mathrm{Dr}-0007 \mathrm{ym}-\mathrm{Hz}$ @example.com

END : VEVENT

END : VCALENDAR

$</$ C:calendar-data $>$

$</ \mathrm{D}:$ prop $>$

$<$ D: status $>$ HTTP/1.1 200 OK</D:status $>$

$</ \mathrm{D}$ :propstat $>$

$</ \mathrm{D}$ :response $>$

$</ D: m u l t i s t a t u s>$ 


\subsubsection{Example: Retrieval of Events by PARTSTAT}

In this example, the client requests the server to return the VEVENT components that have the ATTENDEE property with the value "mailto:lisa@example.com" and for which the PARTSTAT parameter is set to NEEDS-ACTION.

See Appendix B for the calendar data being targeted by this example.

$>$ Request $<<$

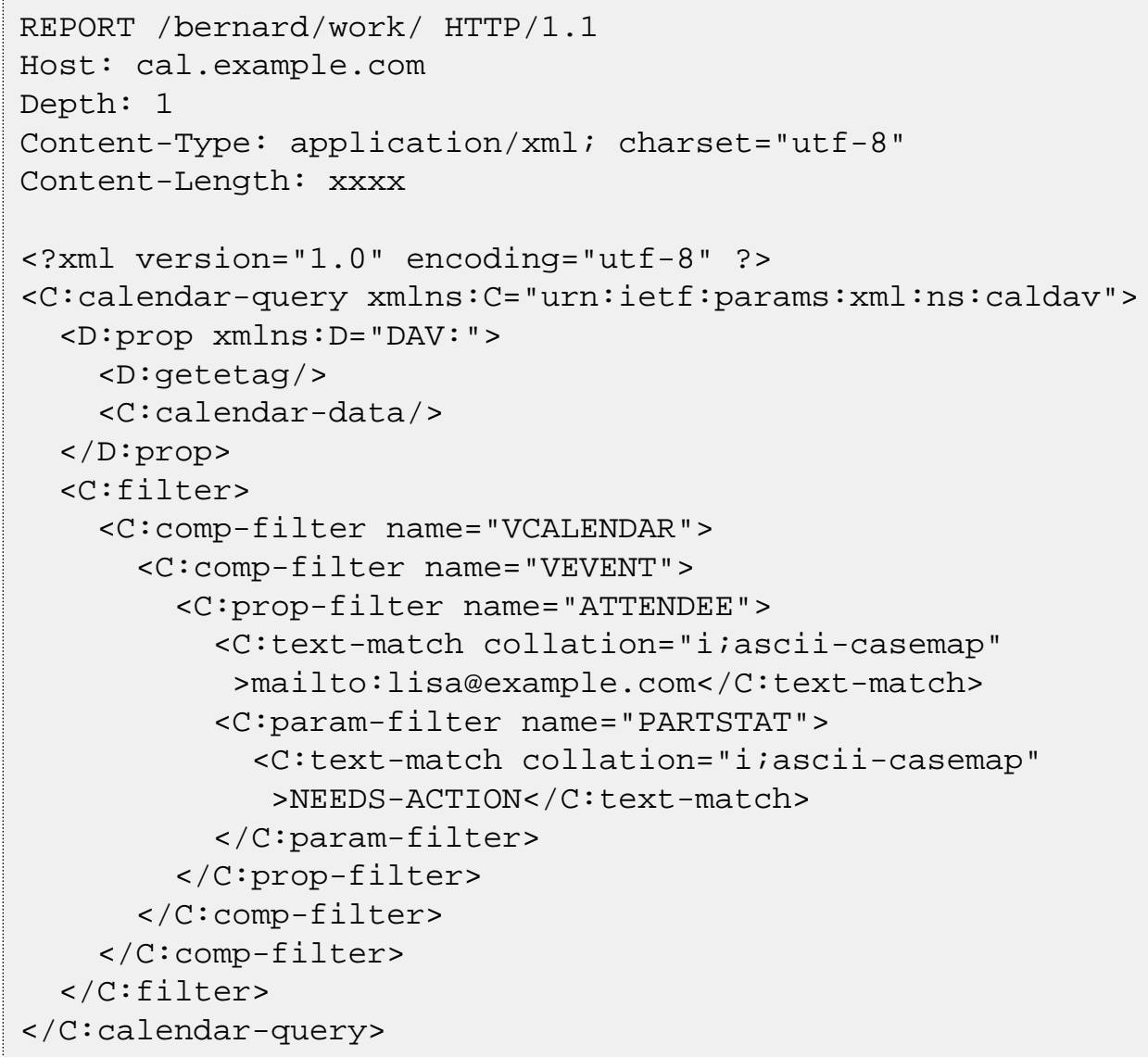




\section{$>$ Response $<<$}

HTTP/1.1 207 Multi-Status

Date: Sat, 11 Nov 2006 09:32:12 GMT

Content-Type: application/xml; charset="utf-8"

Content-Length: $\mathrm{xxxx}$

<?xml version="1.0" encoding="utf-8" ?>

$<$ D:multistatus xmlns:D="DAV:"

xmlns:C="urn: ietf $:$ params: $x m l: n s:$ caldav">

$<\mathrm{D}:$ response>

$<$ D: href $>$ http: //cal . example.com/bernard/work/abcd3.ics</D:href $>$

$<$ D:propstat $>$

$<\mathrm{D}:$ prop $>$

$<$ D : getetag $>$ "fffff-abcd3"</D: getetag $>$

$<$ C:calendar-data>BEGIN : VCALENDAR

VERSION : 2.0

PRODID:-//Example Corp.//CalDAV Client//EN

BEGIN : VTIMEZONE

LAST-MODIFIED : 20040110 T032845Z

TZID:US/Eastern

BEGIN : DAYLIGHT

DTSTART : $20000404 T 020000$

RRULE : $F R E Q=Y E A R L Y$; $B Y D A Y=1 S U$; $B Y M O N T H=4$

TZNAME : EDT

TZOFFSETFROM: -0500

TZOFFSETTO: -0400

END : DAYLIGHT

BEGIN : STANDARD

DTSTART : $20001026 T 020000$

RRULE : $F R E Q=Y E A R L Y ; B Y D A Y=-1 S U$; $B Y M O N T H=10$

TZNAME : EST

TZOFFSETFROM: -0400

TZOFFSETTO:-0500

END : STANDARD

END : VTIMEZONE

BEGIN : VEVENT

ATTENDEE; PARTSTAT=ACCEPTED; ROLE=CHAIR : mailto : cyrusdexample. com

ATTENDEE; PARTSTAT=NEEDS-ACTION : mailto:lisadexample.com

DTSTAMP : $20060206 \mathrm{~T} 001220 \mathrm{Z}$

DTSTART; TZID=US/Eastern:20060104T100000

DURATION : PT $1 \mathrm{H}$

LAST-MODIFIED : 20060206 T001330Z

ORGANIZER:mailto: cyrus@example.com

SEQUENCE : 1

STATUS : TENTATIVE

SUMMARY:Event \#3

UID : DC6C50A017428C5216A2F1CD@example.com

$\mathrm{X}-\mathrm{ABC}-\mathrm{GUID}: \mathrm{E} 1 \mathrm{CX} 5 \mathrm{Dr}-0007 \mathrm{ym}-\mathrm{Hz}$ @example.com

END : VEVENT

END : VCALENDAR

$</$ C:calendar-data $>$

$</ \mathrm{D}:$ prop $>$

$<$ D: status $>$ HTTP/1.1 200 OK</D:status $>$

$</ \mathrm{D}$ :propstat $>$

$</ \mathrm{D}$ :response $>$

$</ D: m u l t i s t a t u s>$ 


\subsubsection{Example: Retrieval of Events Only}

In this example, the client requests the server to return all VEVENT components.

See Appendix B for the calendar data being targeted by this example.

$>$ Request <<

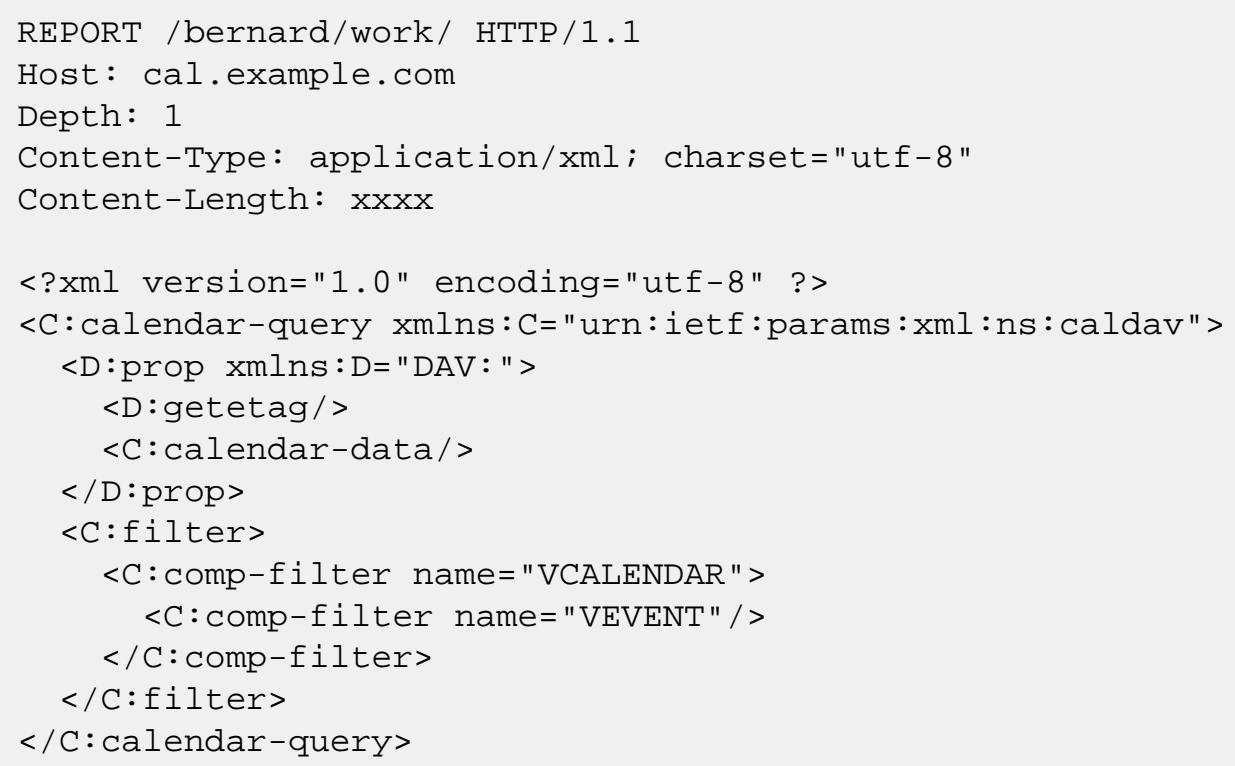




\section{$>$ Response $<<$}

HTTP/1.1 207 Multi-Status

Date: Sat, 11 Nov 2006 09:32:12 GMT

Content-Type: application/xml; charset="utf-8"

Content-Length: $\mathrm{xxxx}$

<?xml version="1.0" encoding="utf-8" ?>

$<$ D:multistatus xmlns:D="DAV:"

xmlns:C="urn: iet $f:$ params: $x m l: n s:$ caldav">

$<\mathrm{D}:$ response>

$<$ D : href>http: / / cal . example.com/bernard/work/abcdl.ics</D: href>

$<\mathrm{D}:$ propstat $>$

$<\mathrm{D}:$ prop $>$

$<$ D : getetag $>$ "fffff-abcd1" </D : getetag $>$

$<$ C:calendar-data>BEGIN : VCALENDAR

VERSION $: 2.0$

PRODID:-//Example Corp.//CalDAV Client//EN

BEGIN : VTIMEZONE

LAST-MODIFIED : 20040110 T032845Z

TZID:US/Eastern

BEGIN : DAYLIGHT

DTSTART : $20000404 T 020000$

RRULE : $F R E Q=Y E A R L Y$; $B Y D A Y=1 S U$; $B Y M O N T H=4$

TZNAME : EDT

TZOFFSETFROM: -0500

TZOFFSETTO: -0400

END :DAYLIGHT

BEGIN : STANDARD

DTSTART : $20001026 T 020000$

RRULE : $F R E Q=Y E A R L Y ; B Y D A Y=-1 S U$; $B Y M O N T H=10$

TZNAME :EST

TZOFFSETFROM: -0400

TZOFFSETTO:-0500

END : STANDARD

END : VTIMEZONE

BEGIN : VEVENT

DTSTAMP :20060206T001102Z

DTSTART; TZID=US/Eastern:20060102T100000

DURATION : PT1H

SUMMARY:Event \#1

Description:Go Steelers!

UID : 74855313 FA803DA593CD579A@example.com

END : VEVENT

END : VCALENDAR

$</ C$ :calendar-data $>$

$</ \mathrm{D}:$ prop $>$

$<$ D:status $>$ HTTP/1.1 200 OK</D:status $>$

$</ \mathrm{D}:$ propstat $>$

$</ D$ :response $>$

$<$ D:response>

$<$ D: href>http://cal.example.com/bernard/work/abcd2.ics</D:href>

$<\mathrm{D}:$ propstat $>$

$<\mathrm{D}:$ prop $>$

$<$ D: getetag>"fffff-abcd2"</D: getetag>

$<$ C:calendar-data>BEGIN:VCALENDAR

VERSION : 2.0

PRODID:-//Example Corp.//CalDAV Client//EN

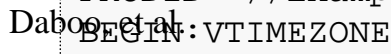

Standards Track

[Page 45]

LAST-MODIFIED : $20040110 T 032845 \mathrm{Z}$ 


\subsubsection{Example: Retrieval of All Pending To-Dos}

In this example, the client requests the server to return all VTODO components that do not include a COMPLETED property and do not have a STATUS property value matching CANCELLED, i.e., VTODOs that still need to be worked on.

See Appendix B for the calendar data being targeted by this example.

$>$ Request $<<$

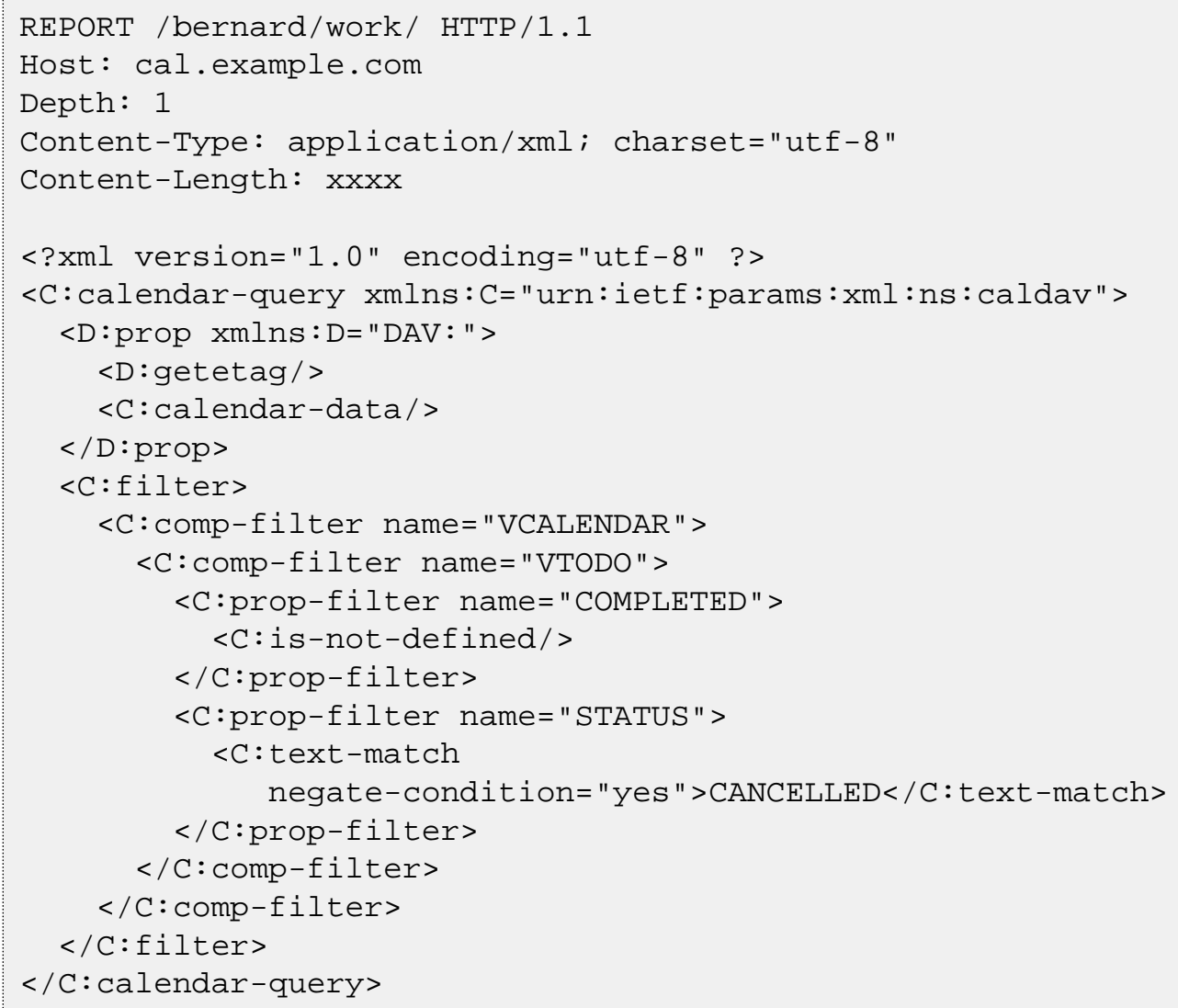




\section{$>$ Response $<<$}

HTTP/1.1 207 Multi-Status

Date: Sat, 11 Nov 2006 09:32:12 GMT

Content-Type: application/xml; charset="utf-8"

Content-Length: $\mathrm{xxxx}$

<?xml version="1.0" encoding="utf-8" ?>

$<$ D:multistatus xmlns:D="DAV:"

xmlns:C="urn: iet $f:$ params: $x m l: n s:$ caldav">

$<\mathrm{D}:$ response>

$<$ D : href>http: / / cal . example.com/bernard/work/abcd4.ics</D: href>

$<\mathrm{D}:$ propstat $>$

$<\mathrm{D}:$ prop $>$

$<$ D : getetag $>$ "fffff-abcd4 "</D: getetag $>$

$<$ C: calendar-data>BEGIN : VCALENDAR

VERSION $: 2.0$

PRODID:-//Example Corp.//CalDAV Client//EN

BEGIN : VTODO

DTSTAMP : $20060205 \mathrm{~T} 235335 \mathrm{Z}$

DUE; VALUE=DATE : 20060104

STATUS : NEEDS-ACTION

SUMMARY:Task \#1

UID : DDDEEB 7915FA61233B861457@example.com

BEGIN : VALARM

ACTION : AUDIO

TRIGGER; RELATED=START : $-\mathrm{PT} 10 \mathrm{M}$

END : VALARM

END : VTODO

END : VCALENDAR

$</ C$ :calendar-data $>$

$</ \mathrm{D}:$ prop $>$

$<$ D:status $>$ HTTP/1.1 200 OK</D:status $>$

$</ D$ :propstat $>$

$</ \mathrm{D}$ :response $>$

$<$ D : response>

$<$ D: href > http: / / cal .example.com/bernard/work/abcd5.ics</D: href $>$

$<\mathrm{D}:$ propstat $>$

$<\mathrm{D}:$ prop $>$

$<$ D : getetag>"fffff-abcd5" </D: getetag $>$

$<$ C:calendar-data>BEGIN : VCALENDAR

VERSION : 2.0

PRODID:-//Example Corp.//CalDAV Client//EN

BEGIN : VTODO

DTSTAMP : $20060205 \mathrm{~T} 235300 \mathrm{Z}$

DUE; VALUE=DATE : 20060106

LAST-MODIFIED : 20060205 T235308Z

SEQUENCE : 1

STATUS : NEEDS-ACTION

SUMMARY:Task \#2

UID : E10BA4 7467C5C69BB74E8720@example.com

BEGIN : VALARM

ACTION : AUDIO

TRIGGER; RELATED=START : $-\mathrm{PT} 10 \mathrm{M}$

END : VALARM

END : VTODO

END : VCALENDAR

Daboe,edala lendar-data>

Standards Track

[Page 47]

</D:prop $>$ 


\subsubsection{Example: Attempt to Query Unsupported Property}

In this example, the client requests the server to return all VEVENT components that include an X-ABC-GUID property with a value matching "ABC". However, the server does not support querying that non-standard property, and instead returns an error response.

See Appendix B for the calendar data being targeted by this example.

$>$ Request $<<$

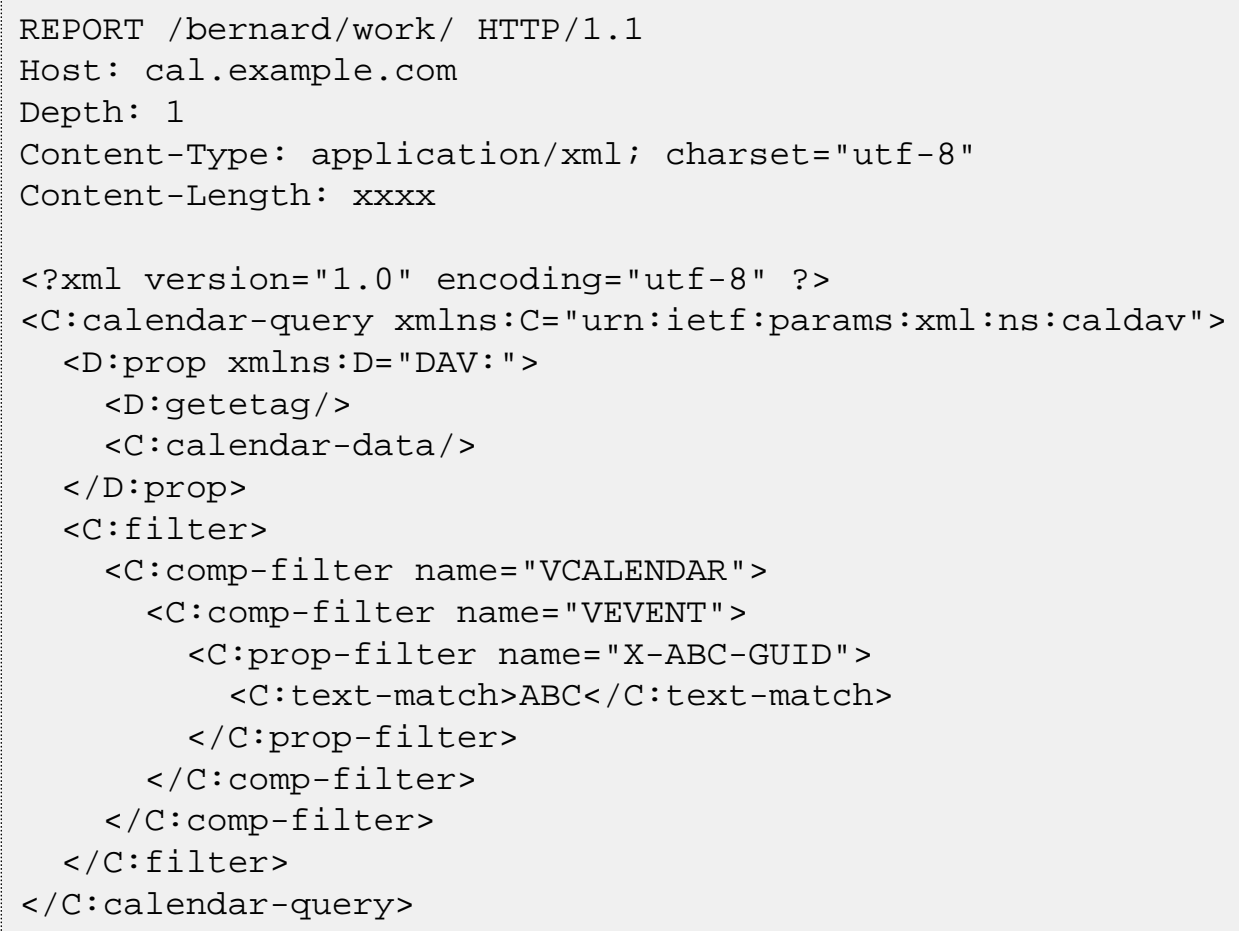

$>$ Response <<

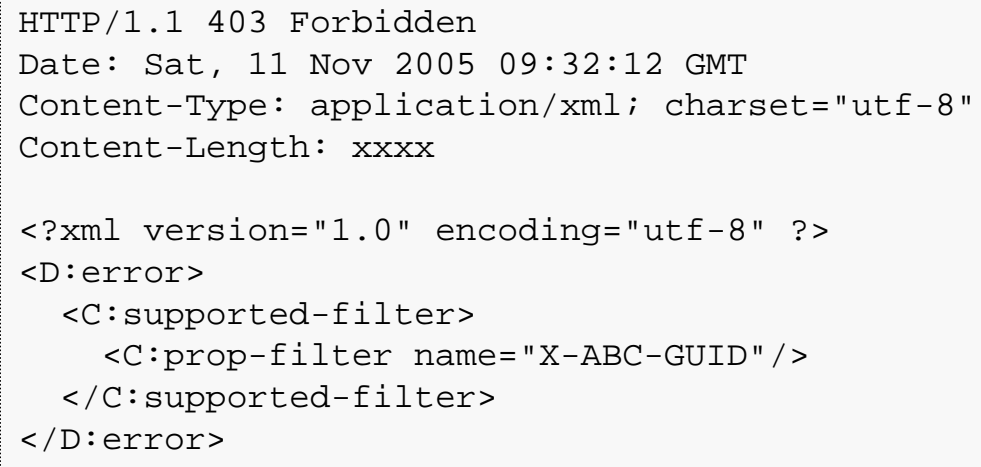

\subsection{CALDAV:calendar-multiget REPORT}

The CALDAV:calendar-multiget REPORT is used to retrieve specific calendar object resources from within a collection, if the Request-URI is a collection, or to retrieve a specific calendar object resource, if the RequestURI is a calendar object resource. This report is similar to the CALDAV:calendar-query REPORT (see Section 7.8), except that it takes a list of DAV:href elements, instead of a CALDAV:filter element, to determine which calendar object resources to return.

Support for the CALDAV:calendar-multiget REPORT is REQUIRED. 


\section{Marshalling:}

The request body MUST be a CALDAV:calendar-multiget XML element (see Section 9.10). If the Request-URI is a collection resource, then the DAV:href elements MUST refer to calendar object resources within that collection, and they MAY refer to calendar object resources at any depth within the collection. As a result, the "Depth" header MUST be ignored by the server and SHOULD NOT be sent by the client. If the Request-URI refers to a non-collection resource, then there MUST be a single DAV:href element that is equivalent to the Request-URI.

The response body for a successful request MUST be a DAV:multistatus XML element.

The response body for a successful CALDAV:calendar-multiget REPORT request MUST contain a DAV:response element for each calendar object resource referenced by the provided set of DAV:href elements. Calendar data is being returned in the CALDAV:calendar-data element inside the DAV:prop element.

In the case of an error accessing any of the provided DAV:href resources, the server MUST return the appropriate error status code in the DAV:status element of the corresponding DAV:response element.

\section{Preconditions:}

(CALDAV:supported-calendar-data): The attributes "content-type" and "version" of the

CALDAV:calendar-data XML elements (see Section 9.6) specify a media type supported by the server for calendar object resources.

(CALDAV:min-date-time): Any XML element specifying a range of time MUST have its start or end DATE or DATE-TIME values greater than or equal to the value of the CALDAV:min-date-time property value (Section 5.2.6) on the calendar collections being targeted by the REPORT request;

(CALDAV:max-date-time): Any XML element specifying a range of time MUST have its start or end DATE or DATE-TIME values less than or equal to the value of the CALDAV:max-date-time property value (Section 5.2.7) on the calendar collections being targeted by the REPORT request;

\section{Postconditions:}

None.

\subsubsection{Example: Successful CALDAV:calendar-multiget REPORT}

In this example, the client requests the server to return specific properties of the VEVENT components referenced by specific URIs. In addition, the DAV:getetag property is also requested and returned as part of the response. Note that in this example, the resource at http://cal.example.com/bernard/work/mtg 1 ics does not exist, resulting in an error status response.

See Appendix B for the calendar data being targeted by this example. 


\section{> Request $<<$}

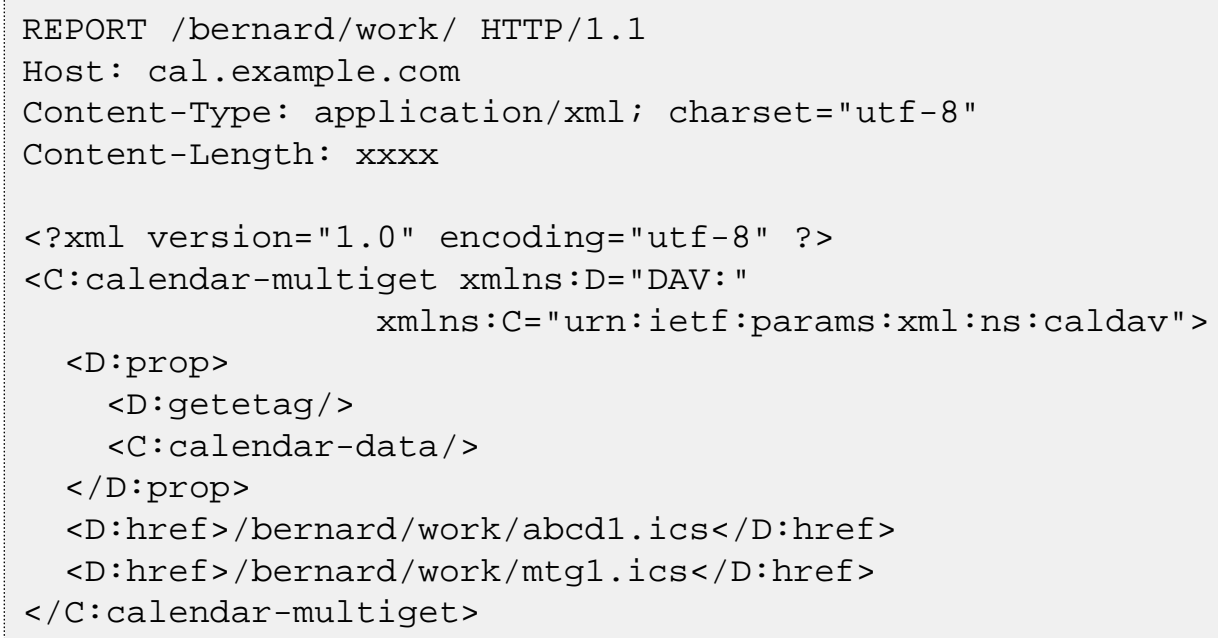




\section{$>$ Response $<<$}

HTTP/1.1 207 Multi-Status

Date: Sat, 11 Nov 2006 09:32:12 GMT

Content-Type: application/xml; charset="utf-8"

Content-Length: $\mathrm{xxxx}$

<?xml version="1.0" encoding="utf-8" ?>

$<$ D:multistatus xmlns:D="DAV:"

xmlns:C="urn: ietf $:$ params: $x m l: n s:$ caldav">

$<\mathrm{D}:$ response>

<D: href>http://cal.example.com/bernard/work/abcdl.ics</D:href>

$<$ D:propstat $>$

$<\mathrm{D}:$ prop $>$

$<$ D : getetag $>$ "fffff-abcd1" </D : getetag $>$

$<$ C:calendar-data>BEGIN : VCALENDAR

VERSION : 2.0

PRODID:-//Example Corp.//CalDAV Client//EN

BEGIN : VTIMEZONE

LAST-MODIFIED : 20040110 T032845Z

TZID:US/Eastern

BEGIN : DAYLIGHT

DTSTART : $20000404 T 020000$

RRULE : $F R E Q=Y E A R L Y ; B Y D A Y=1 S U$; $B Y M O N T H=4$

TZNAME : EDT

TZOFFSETFROM: -0500

TZOFFSETTO: -0400

END : DAYLIGHT

BEGIN : STANDARD

DTSTART : $20001026 T 020000$

RRULE : $F R E Q=Y E A R L Y ; B Y D A Y=-1 S U$; $B Y M O N T H=10$

TZNAME :EST

TZOFFSETFROM: -0400

TZOFFSETTO: -0500

END : STANDARD

END : VTIMEZONE

BEGIN : VEVENT

DTSTAMP : $20060206 \mathrm{~T} 001102 \mathrm{Z}$

DTSTART; TZID=US/Eastern:20060102T100000

DURATION : PT1H

SUMMARY:Event \#1

Description:Go Steelers!

UID : 74855313 FA803DA593CD579A@example.com

END : VEVENT

END:VCALENDAR

$</ \mathrm{C}$ :calendar-data $>$

$</ \mathrm{D}:$ prop $>$

$<$ D:status $>$ HTTP/1.1 200 OK</D:status $>$

$</ \mathrm{D}:$ propstat $>$

$</ D$ : response>

$<$ D:response>

$<$ D: href $>$ http://cal. example.com/bernard/work/mtg1.ics</D:href $>$

$<$ D:status $>$ HTTP/1.1 404 Not Found</D:status $>$

$</ D$ :response $>$

$</ D$ :multistatus $>$ 


\subsection{CALDAV:free-busy-query REPORT}

The CALDAV:free-busy-query REPORT generates a VFREEBUSY component containing free busy information for all the calendar object resources targeted by the request and that have the CALDAV:read-freebusy or DAV:read privilege granted to the current user.

Only VEVENT components without a TRANSP property or with the TRANSP property set to OPAQUE, and VFREEBUSY components SHOULD be considered in generating the free busy time information.

In the case of VEVENT components, the free or busy time type (FBTYPE) of the FREEBUSY properties in the returned VFREEBUSY component SHOULD be derived from the value of the TRANSP and STATUS properties, as outlined in the table below:

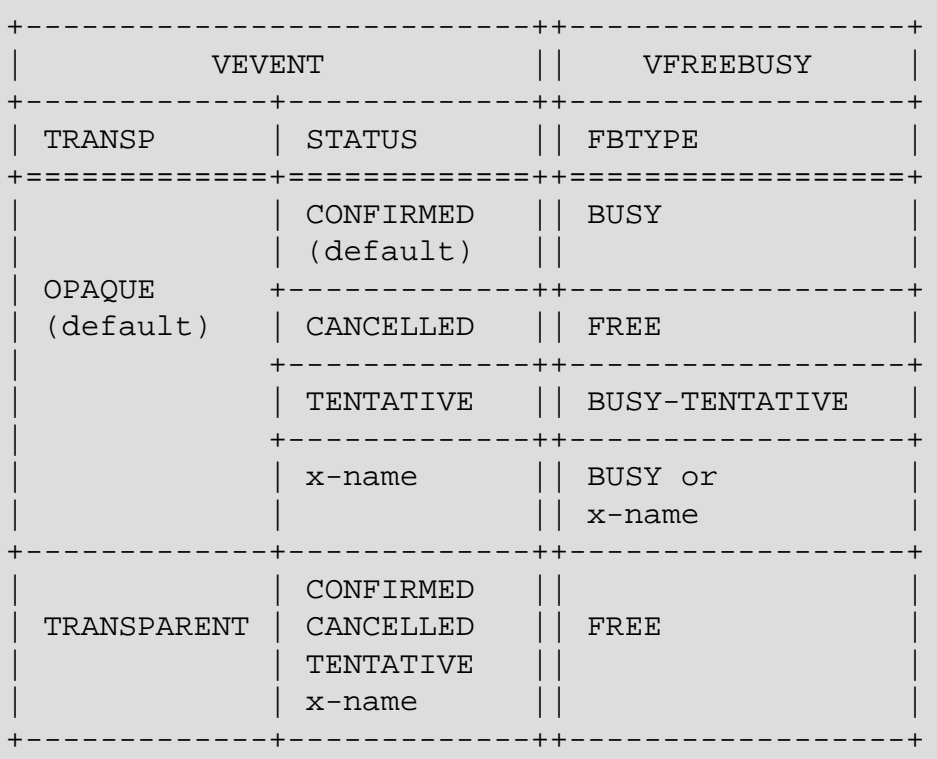

Duplicate busy time periods with the same FBTYPE parameter value SHOULD NOT be specified in the returned VFREEBUSY component. Servers SHOULD coalesce consecutive or overlapping busy time periods of the same type. Busy time periods with different FBTYPE parameter values MAY overlap.

Support for the CALDAV:free-busy-query REPORT is REQUIRED.

\section{Marshalling:}

The request body MUST be a CALDAV:free-busy-query XML element (see Section 9.11), which MUST contain exactly one CALDAV:time-range XML element, as defined in Section 9.9.

The request MAY include a Depth header. If no Depth header is included, Depth:0 is assumed.

The response body for a successful request MUST be an iCalendar object that contains exactly one VFREEBUSY component that describes the busy time intervals for the calendar object resources containing VEVENT, or VFREEBUSY components that satisfy the Depth value and for which the current user is at least granted the CALDAV:read-free-busy privilege. If no calendar object resources are found to satisfy these conditions, a VFREEBUSY component with no FREEBUSY property MUST be returned. This report only returns busy time information. Free time information can be inferred from the returned busy time information.

If the current user is not granted the CALDAV:read-free-busy or DAV:read privileges on the RequestURI, the CALDAV:free-busy-query REPORT request MUST fail and return a 404 (Not Found) status value. This restriction will prevent users from discovering URLs of resources for which they are only granted the CALDAV:read-free-busy privilege. 
The CALDAV:free-busy-query REPORT request can only be run against a collection (either a regular collection or a calendar collection). An attempt to run the report on a calendar object resource MUST fail and return a 403 (Forbidden) status value.

\section{Preconditions:}

None.

\section{Postconditions:}

(DAV:number-of-matches-within-limits): The number of matching calendar object resources must fall within server-specific, predefined limits. For example, this postcondition might fail if the specified CALDAV:time-range would cause an extremely large number of calendar object resources to be considered in computing the response.

\subsubsection{Example: Successful CALDAV:free-busy-query REPORT}

In this example, the client requests the server to return free busy information on the calendar collection / bernard/work/, between 9:00 A.M. and 5:00 P.M. EST (2:00 P.M. and 10:00 P.M. UTC) on the January 4, 2006. The server responds, indicating two busy time intervals of one hour, one of which is tentative.

See Appendix B for the calendar data being targeted by this example.

$>>$ Request $<$

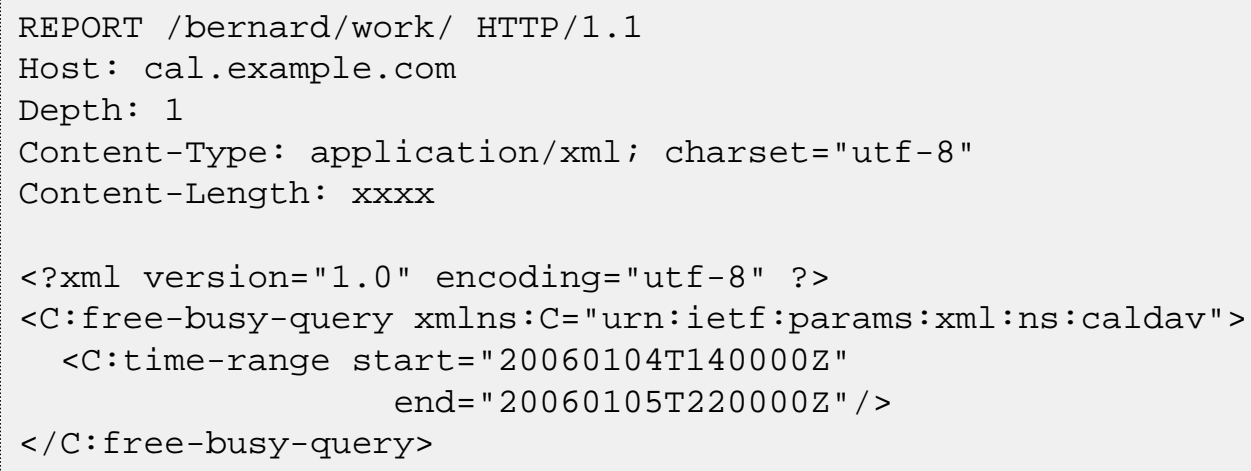




\section{Guidelines}

\subsection{Client-to-Client Interoperability}

There are a number of actions clients can take that will be legal (the server will not return errors), but that can degrade interoperability with other client implementations accessing the same data. For example, a recurrence rule could be replaced with a set of recurrence dates, a single recurring event could be replaced with a set of independent resources to represent each recurrence, or the start/end time values can be translated from the original time zone to another time zone. Although this advice amounts to iCalendar interoperability best practices and is not limited only to CalDAV usage, interoperability problems are likely to be more evident in CalDAV use cases.

\subsection{Synchronization Operations}

WebDAV already provides functionality required to synchronize a collection or set of collections, to make changes offline, and provides a simple way to resolve conflicts when reconnected. ETags are the key to making this work, but these are not required of all WebDAV servers. Since offline functionality is more important to calendar applications than to some other WebDAV applications, CalDAV servers MUST support ETags, as specified in Section 5.3.4.

\subsubsection{Use of Reports}

\subsubsection{Restrict the Time Range}

The reports provided in CalDAV can be used by clients to optimize their performance in terms of network bandwidth usage and resource consumption on the local client machine. Both are certainly major considerations for mobile or handheld devices with limited capacity, but they are also relevant to desktop client applications in cases where the calendar collections contain large amounts of data.

Typically, clients present calendar data to users in views that span a finite time interval, so whenever possible, clients should only retrieve calendar components from the server using CALDAV:calendar-query REPORT, combined with a CALDAV:time-range element, to limit the set of returned components to just those needed to populate the current view.

\subsubsection{Synchronize by Time Range}

Typically in a calendar, historical data (events, to-dos, etc. that have completed prior to the current date) do not change, though they may be deleted. As a result, a client can speed up the synchronization process by only considering data for the present time and the future up to a reasonable limit (e.g., one week, one month). If the user then tries to examine a portion of the calendar outside the range that has been synchronized, the client can perform another synchronization operation on the new time interval being examined. This "just-in-time" synchronization can minimize bandwidth for common user interaction behaviors.

\subsubsection{Synchronization Process}

If a client wants to support calendar data synchronization, as opposed to downloading calendar data each time it is needed, the client needs to cache the calendar object resource's URI and ETag, along with the actual calendar data. While the URI remains static for the lifetime of the calendar object resource, the ETag will change with each successive change to the calendar object resource. Thus, to synchronize a local data cache with the server, the client can first fetch the URI/ETag pairs for the time interval being considered, and compare those results with the cached data. Any cached component whose ETag differs from that on the server needs to be refreshed.

In order to properly detect the changes between the server and client data, the client will need to keep a record of which calendar object resources have been created, changed, or deleted since the last synchronization operation so that it can reconcile those changes with the data on the server.

Here's an example of how to do that: 
The client issues a CALDAV:calendar-query REPORT request for a specific time range and asks for only the DAV:getetag property to be returned:

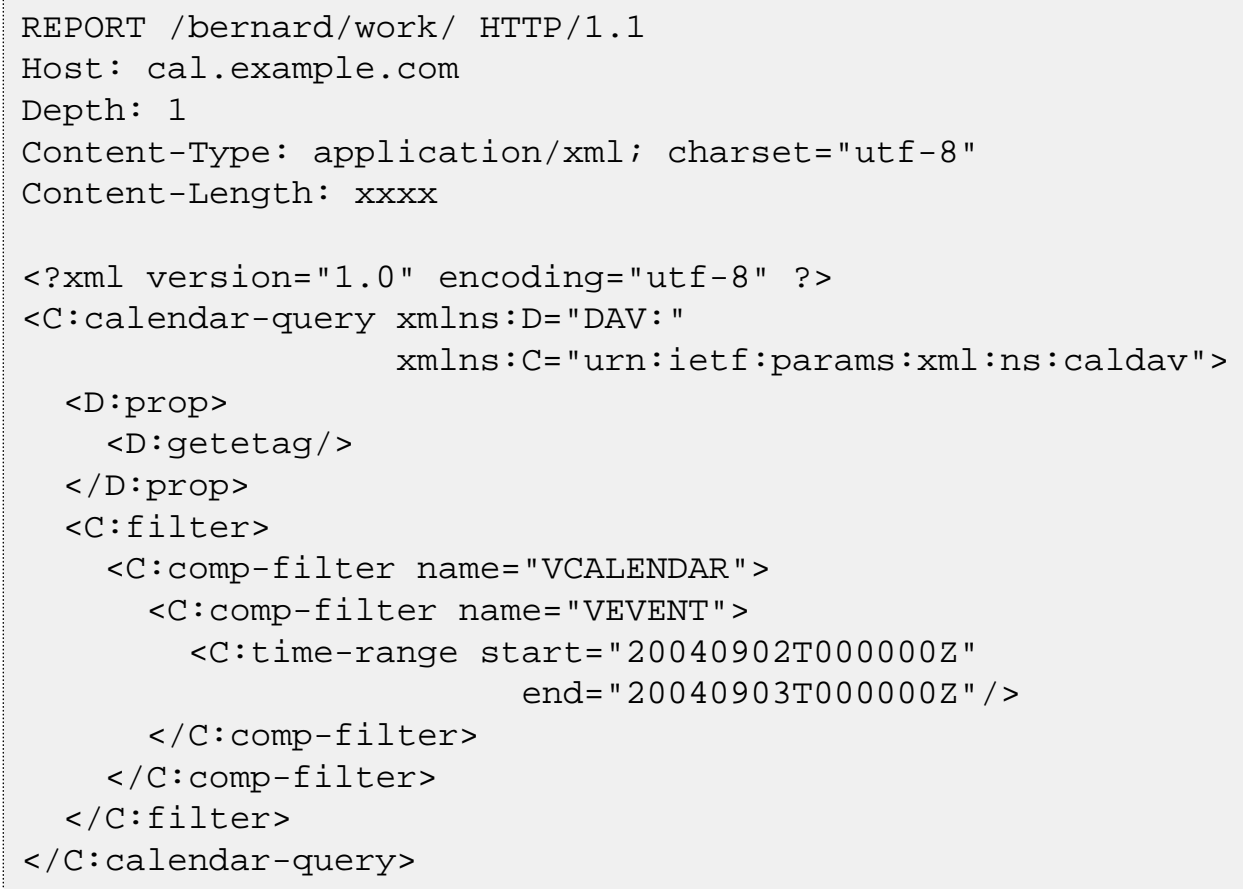

The client then uses the results to determine which calendar object resources have changed, been created, or deleted on the server, and how those relate to locally cached calendar object resources that may have changed, been created, or deleted. If the client determines that there are calendar object resources on the server that need to be fetched, the client issues a CALDAV:calendar-multiget REPORT request to fetch its calendar data:

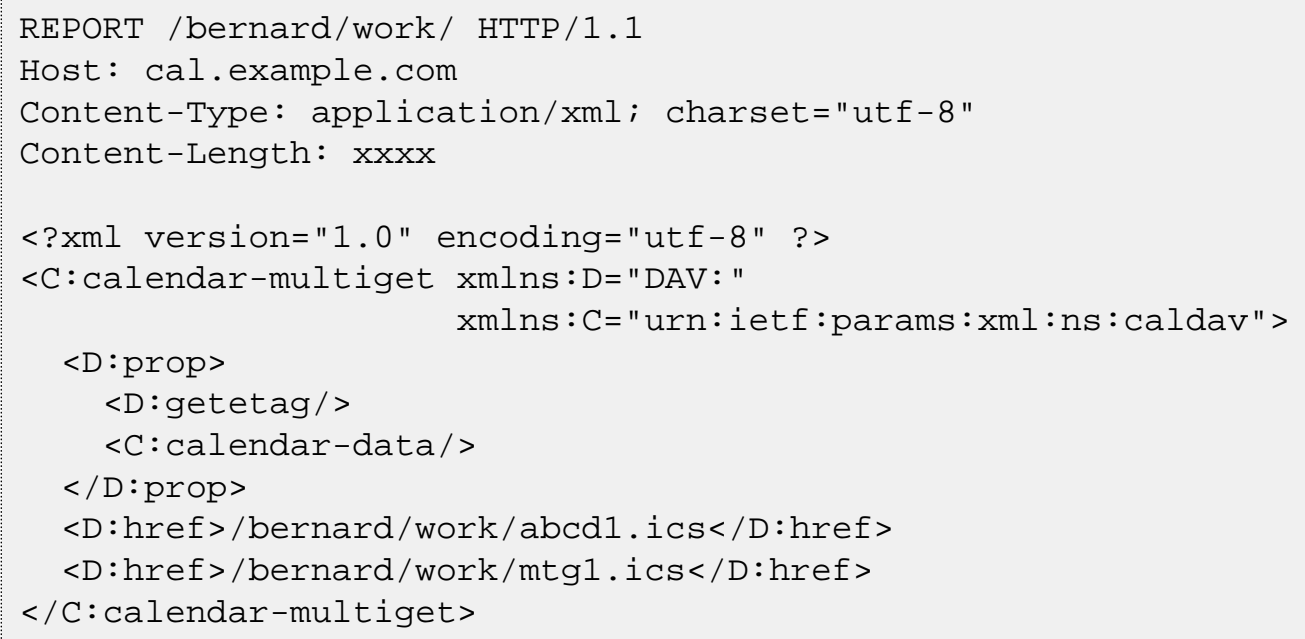

\subsubsection{Restrict the Properties Returned}

A client may not need all the calendar properties of a calendar object resource when presenting information to the user. Since some calendar property values can be large (e.g., ATTACH or ATTENDEE), a client can choose to restrict the calendar properties to be returned in a calendaring REPORT request to those it knows it will use.

However, if a client needs to make a change to a calendar object resource, it can only change the entire calendar object resource via a PUT request. There is currently no way to incrementally make a change to a set 
of calendar properties of a calendar object resource. As a result, the client will have to get the entire calendar object resource that is being changed.

\subsection{Use of Locking}

WebDAV locks can be used to prevent two clients that are modifying the same resource from either overwriting each others' changes (though that problem can also be solved by using ETags) or wasting time making changes that will conflict with another set of changes. In a multi-user calendar system, an interactive calendar client could lock an event while the user is editing the event, and unlock the event when the user finishes or cancels. Locks can also be used to prevent changes while data is being reorganized. For example, a calendar client might lock two calendar collections prior to moving a bunch of calendar resources from one to another.

Clients are responsible for requesting a lock timeout period that is appropriate to the use case. When the user explicitly decides to reserve a resource and prevent other changes, a long timeout might be appropriate, but in cases where the client automatically decides to lock the resource, the timeout should be short (and the client can always refresh the lock should it need to). A short lock timeout means that if the client is unable to remove the lock, the other calendar users aren't prevented from making changes.

\subsection{Finding Calendars}

Much of the time, a calendar client (or agent) will discover a new calendar's location by being provided directly with the URL. For example, a user will type his or her own calendar location into client configuration information or copy and paste a URL from email into the calendar application. The client need only confirm that the URL points to a resource that is a calendar collection. The client may also be able to browse WebDAV collections to find calendar collections.

The choice of HTTP URLs means that calendar object resources are backward compatible with existing software, but does have the disadvantage that existing software does not usually know to look at the OPTIONS response to that URL to determine what can be done with it. This is somewhat of a barrier for WebDAV usage as well as with CalDAV usage. This specification does not offer a way through this other than making the information available in the OPTIONS response should this be requested.

For calendar sharing and scheduling use cases, one might wish to find the calendar belonging to another user. If the other user has a calendar in the same repository, that calendar can be found by using the principal namespace required by WebDAV ACL support. For other cases, the authors have no universal solution, but implementers can consider whether to use vCard [RFC2426] or LDAP [RFC4511] standards together with calendar attributes [RFC2739].

Because CalDAV requires servers to support WebDAV ACL [RFC3744], including principal namespaces, and with the addition of the CALDAV:calendar-home-set property, there are a couple options for CalDAV clients to find one's own calendar or another user's calendar.

In this case, a DAV:principal-match REPORT is used to find a named property (the CALDAV:calendar-homeset) on the Principal-URL of the current user. Using this, a WebDAV client can learn "who am I" and "where are my calendars". The REPORT request body looks like this:

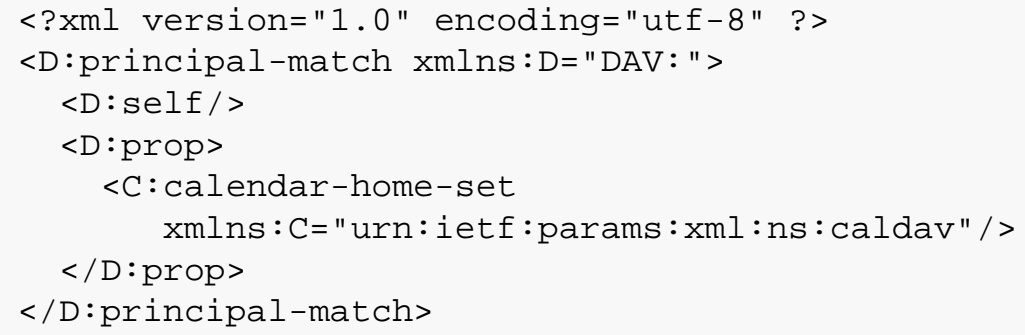


To find other users' calendars, the DAV:principal-property-search REPORT can be used to filter on some properties and return others. To search for a calendar owned by a user named "Laurie", the REPORT request body would look like this:

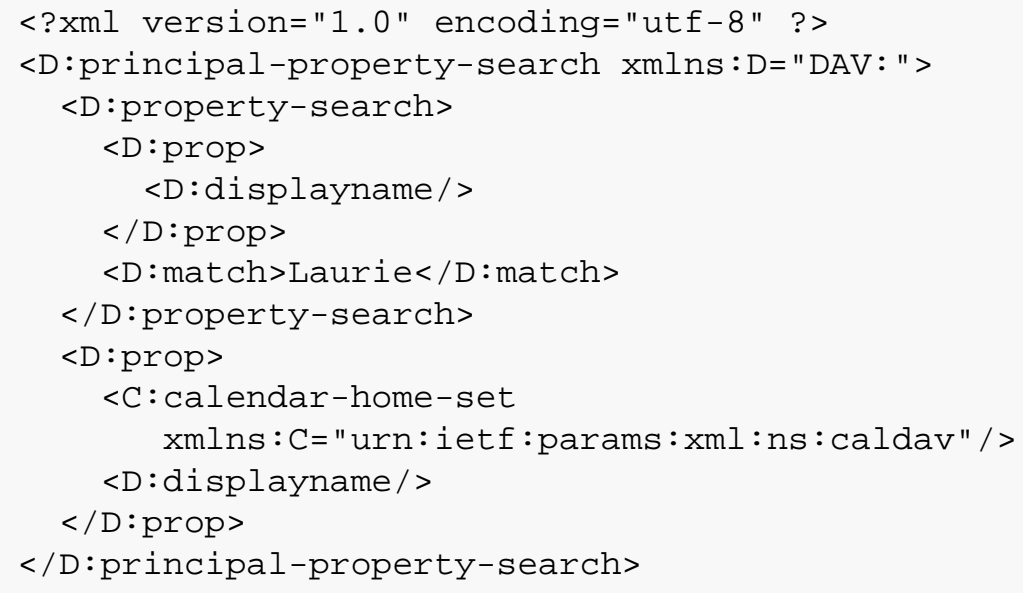

The server performs a case-sensitive or caseless search for a matching string subset of "Laurie" within the DAV:displayname property. Thus, the server might return "Laurie Dusseault", "Laurier Desruisseaux", or

"Wilfrid Laurier" as matching DAV:displayname values, and return the calendars for each of these.

\subsection{Storing and Using Attachments}

CalDAV clients MAY create attachments in calendar components either as inline or external. This section contains some guidelines for creating and managing attachments.

\subsubsection{Inline Attachments}

CalDAV clients MUST support inline attachments as specified in iCalendar [RFC2445]. CalDAV servers MUST support inline attachments, so clients can rely on being able to create attachments this way. On the other hand, inline attachments have some drawbacks:

- Servers MAY impose limitations on the size of calendar object resources (i.e., refusing PUT requests of very large iCalendar objects). Servers that impose such limitations MUST use the CALDAV:max-resourcesize property on a calendar collection to inform the client as to what the limitation is (see Section 5.2.5).

- Servers MAY impose storage quota limitations on calendar collections (See [RFC4331]).

- Any change to a calendar object resource containing an inline attachment requires the entire inline attachment to be re-uploaded.

- Clients synchronizing a changed calendar object resource have to download the entire calendar object resource, even if the attachment is unchanged.

\subsubsection{External Attachments}

CalDAV clients SHOULD support downloading of external attachments referenced by arbitrary URI schemes, by either processing them directly, or by passing the attachment URI to a suitable "helper application" for processing, if such an application exists. CalDAV clients MUST support downloading of external attachments referenced by the "http" or "https" URI schemes. An external attachment could be:

- In a collection in the calendar collection containing the calendar object resource;

- Somewhere else in the same repository that hosts the calendar collection; or

- On an HTTP or FTP server elsewhere.

CalDAV servers MAY provide support for child collections in calendar collections. CalDAV servers MAY allow the MKCOL method to create child collections in calendar collections. Child collections of calendar 
collections MAY contain any type of resource except calendar collections that they MUST NOT contain. Some CalDAV servers won't allow child collections in calendar collections, and it may be possible on such a server to discover other locations where attachments can be stored.

Clients are entirely responsible for maintaining reference consistency with calendar components that link to external attachments. A client deleting a calendar component with an external attachment might therefore also delete the attachment if that's appropriate; however, appropriateness can be very hard to determine. A new component might easily reference some pre-existing Web resource that is intended to have independent existence from the calendar component (the "attachment" could be a major proposal to be discussed in a meeting, for instance). Best practices will probably emerge and should probably be documented, but for now, clients should be wary of engaging in aggressive "cleanup" of external attachments. A client could involve the user in making decisions about removing unreferenced documents, or a client could be conservative in only deleting attachments it had created.

Also, clients are responsible for consistency of permissions when using external attachments. One reason for servers to support the storage of attachments within child collections of calendar collections is that ACL inheritance might make it easier to grant the same permissions to attachments that are granted on the calendar collection. Otherwise, it can be very difficult to keep permissions synchronized. With attachments stored on separate repositories, it can be impossible to keep permissions consistent -- the two repositories may not support the same permissions or have the same set of principals. Some systems have used tickets or other anonymous access control mechanisms to provide partially satisfactory solutions to these kinds of problems.

\subsection{Storing and Using Alarms}

Note that all CalDAV calendar collections (including those the user might treat as public or group calendars) can contain alarm information on events and to-dos. Users can synchronize a calendar between multiple devices and decide to have alarms execute on a different device than the device that created the alarm. Not all alarm action types are completely interoperable (e.g., those that name a sound file to play).

When the action is AUDIO and the client is configured to execute the alarm, the client SHOULD play the suggested sound if it's available or play another sound, but SHOULD NOT rewrite the alarm just to replace the suggested sound with a sound that's locally available.

When the action is DISPLAY and the client is configured to execute the alarm, the client SHOULD execute a display alarm by displaying according to the suggested description or some reasonable replacement, but SHOULD NOT rewrite the alarm for its own convenience.

When the action is EMAIL and the client is incapable of sending email, it SHOULD ignore the alarm, but it MUST continue to synchronize the alarm itself.

This specification makes no recommendations about executing alarms of type PROCEDURE, except to note that clients are advised to take care to avoid creating security holes by executing these.

Non-interoperable alarm information (e.g., should somebody define a color to be used in a display alarm) should be put in non-standard properties inside the VALARM component in order to keep the basic alarm usable on all devices.

Clients that allow changes to calendar object resources MUST synchronize the alarm data that already exists in the resources. Clients MAY execute alarms that are downloaded in this fashion, possibly based on user preference. If a client is only doing read operations on a calendar and there is no risk of losing alarm information, then the client MAY discard alarm information.

This specification makes no attempt to provide multi-user alarms on group calendars or to find out for whom an alarm is intended. Addressing those issues might require extensions to iCalendar; for example, to store alarms per-user, or to indicate for which user a VALARM was intended. In the meantime, clients might maximize interoperability by generally not uploading alarm information to public, group, or resource calendars. 


\section{XML Element Definitions}

\subsection{CALDAV:calendar XML Element}

Name:

Namespace:

Purpose:

Description:

Definition: calendar

urn:ietf:params:xml:ns:caldav

Specifies the resource type of a calendar collection.

See Section 4.2.

$<$ ! ELEMENT calendar EMPTY>

\subsection{CALDAV:mkcalendar XML Element}

Name:

Namespace:

Purpose:

Description:

Definition: mkcalendar

urn:ietf:params:xml:ns:caldav

Specifies a request that includes the WebDAV property values to be set for a calendar collection resource when it is created.

See Section 5.3.1.

\subsection{CALDAV:mkcalendar-response XML Element}

Name: $\quad$ mkcalendar-response

Namespace: $\quad$ urn:ietf:params:xml:ns:caldav

Purpose: $\quad$ Specifies a response body for a successful MKCALENDAR request.

Description: $\quad$ See Section 5.3.1.

Definition: $\quad$ <!ELEMENT mkcalendar-response ANY>

\subsection{CALDAV:supported-collation XML Element}

Name:

Namespace:

Purpose:

Description:

Definition: supported-collation

urn:ietf:params:xml:ns:caldav

Identifies a single collation via its collation identifier, as defined by [RFC4790].

The CALDAV:supported-collation contains the text of a collation identifier, as described in Section 7.5.1.
<!ELEMENT supported-collation (\#PCDATA) >

PCDATA value: collation identifier

\subsection{CALDAV:calendar-query XML Element}

Name:

Namespace:

Purpose:

Description: calendar-query

urn:ietf:params:xml:ns:caldav

Defines a report for querying calendar object resources.

See Section 7.8. 
Definition:

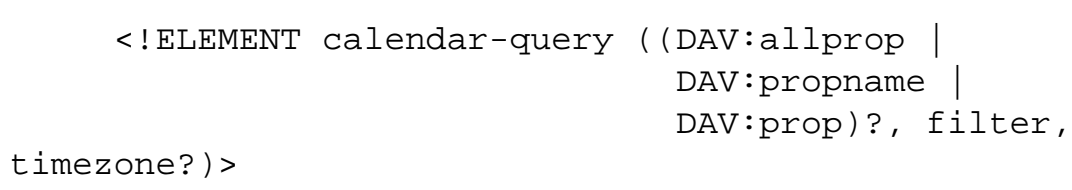

\subsection{CALDAV:calendar-data XML Element}

Name:

Namespace:

Purpose:

Description:

Note:

Note: calendar-data

urn:ietf:params:xml:ns:caldav

Specified one of the following:

1. A supported media type for calendar object resources when nested in the CALDAV:supported-calendar-data property;

2. The parts of a calendar object resource should be returned by a calendaring report;

3. The content of a calendar object resource in a response to a calendaring report.

When nested in the CALDAV:supported-calendar-data property, the CALDAV:calendar-data XML element specifies a media type supported by the CalDAV server for calendar object resources.

When used in a calendaring REPORT request, the CALDAV:calendar-data XML element specifies which parts of calendar object resources need to be returned in the response. If the CALDAV:calendar-data XML element doesn't contain any CALDAV:comp element, calendar object resources will be returned in their entirety.

Finally, when used in a calendaring REPORT response, the CALDAV:calendar-data XML element specifies the content of a calendar object resource. Given that XML parsers normalize the two-character sequence CRLF (US-ASCII decimal 13 and USASCII decimal 10) to a single LF character (US-ASCII decimal 10), the CR character (US-ASCII decimal 13) MAY be omitted in calendar object resources specified in the CALDAV:calendar-data XML element. Furthermore, calendar object resources specified in the CALDAV:calendar-data XML element MAY be invalid per their media type specification if the CALDAV:calendar-data XML element part of the calendaring REPORT request did not specify required properties (e.g., UID, DTSTAMP, etc.), or specified a CALDAV:prop XML element with the "novalue" attribute set to "yes".

The CALDAV:calendar-data XML element is specified in requests and responses inside the DAV:prop XML element as if it were a WebDAV property. However, the CALDAV:calendar-data XML element is not a WebDAV property and, as such, is not returned in PROPFIND responses, nor used in PROPPATCH requests.

The iCalendar data embedded within the CALDAV:calendar-data XML element MUST follow the standard XML character data encoding rules, including use of \&lt; \&gt; \&amp; etc. entity encoding or the use of a $<$ ![CDATA $[. .].]>$ construct. In the later case, the iCalendar data cannot contain the character sequence "]]>", which is the end delimiter for the CDATA section. 
Definition:

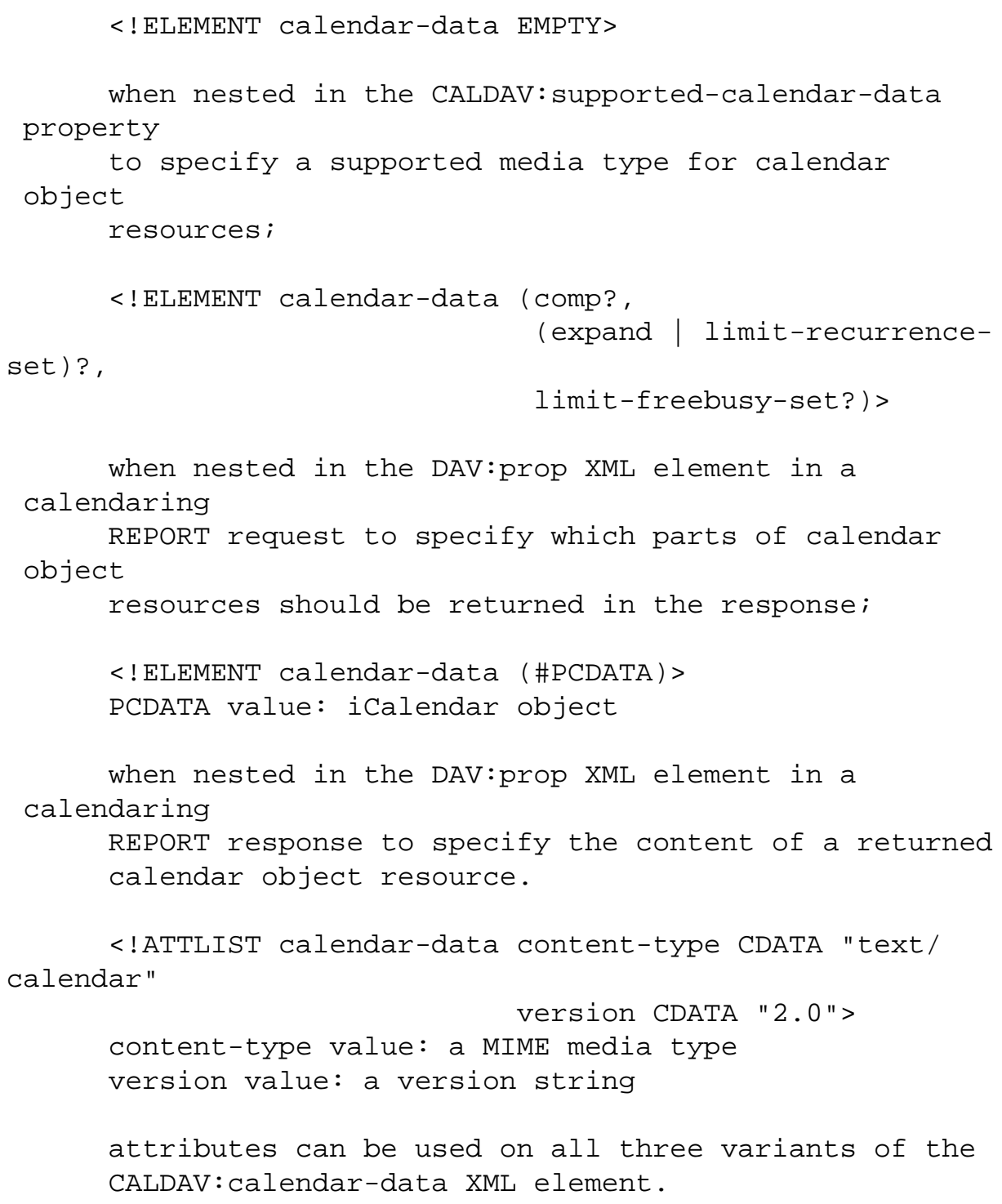

\subsubsection{CALDAV:comp XML Element}

Name:

Namespace:

Purpose:

Description:

Definition:

Note: comp

urn:ietf:params:xml:ns:caldav

Defines which component types to return.

The name value is a calendar component name (e.g., VEVENT).

$$
\begin{aligned}
& \text { comp*) })> \\
& <\text { !ATTLIST comp name CDATA \#REQUIRED> } \\
& \text { name value: a calendar component name }
\end{aligned}
$$$$
\text { <!ELEMENT comp ((allprop | prop*), (allcomp | }
$$

The CALDAV:prop and CALDAV:allprop elements have the same name as the DAV:prop and DAV:allprop elements defined in [RFC2518]. However, 
the CALDAV:prop and CALDAV:allprop elements are defined in the "urn:ietf:params:xml:ns:caldav" namespace instead of the "DAV:" namespace.

\subsubsection{CALDAV:allcomp XML Element}

Name:

Namespace:

Purpose:

Description:

Definition:

\section{allcomp}

urn:ietf:params:xml:ns:caldav

Specifies that all components shall be returned.

The CALDAV:allcomp XML element can be used when the client wants all types of components returned by a calendaring REPORT request.

$$
<\text { !ELEMENT allcomp EMPTY> }
$$

\subsubsection{CALDAV:allprop XML Element}

Name:

Namespace:

Purpose:

Description:

Definition:

Note: allprop

urn:ietf:params:xml:ns:caldav

Specifies that all properties shall be returned.

The CALDAV:allprop XML element can be used when the client wants all properties of components returned by a calendaring REPORT request.

$$
<\text { !ELEMENT allprop EMPTY> }
$$

The CALDAV:allprop element has the same name as the DAV:allprop element defined in [RFC2518]. However, the CALDAV:allprop element is defined in the "urn:ietf:params:xml:ns:caldav" namespace instead of the "DAV:" namespace.

\subsubsection{CALDAV:prop XML Element}

Name:

Namespace:

Purpose:

Description:

Definition:

Note: prop

urn:ietf:params:xml:ns:caldav

Defines which properties to return in the response.

The "name" attribute specifies the name of the calendar property to return (e.g., ATTENDEE). The "novalue" attribute can be used by clients to request that the actual value of the property not be returned (if the "novalue" attribute is set to "yes"). In that case, the server will return just the iCalendar property name and any iCalendar parameters and a trailing ":" without the subsequent value data.

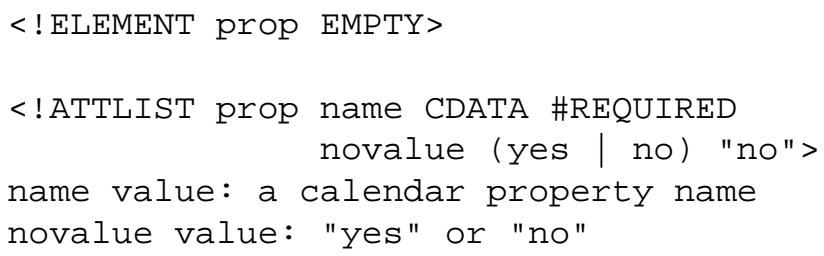

The CALDAV:prop element has the same name as the DAV:prop element defined in [RFC2518]. However, the CALDAV:prop element is defined in the "urn:ietf:params:xml:ns:caldav" namespace instead of the "DAV:" namespace.

\subsubsection{CALDAV:expand XML Element}

Name:

expand 
Namespace: $\quad$ urn:ietf:params:xml:ns:caldav

Purpose:

Description:

Definition:
Forces the server to expand recurring components into individual recurrence instances.

The CALDAV:expand XML element specifies that for a given calendaring REPORT request, the server MUST expand the recurrence set into calendar components that define exactly one recurrence instance, and MUST return only those whose scheduled time intersect a specified time range.

The "start" attribute specifies the inclusive start of the time range, and the "end" attribute specifies the non-inclusive end of the time range. Both attributes are specified as date with UTC time value. The value of the "end" attribute MUST be greater than the value of the "start" attribute.

The server MUST use the same logic as defined for CALDAV:time-range to determine if a recurrence instance intersects the specified time range.

Recurring components, other than the initial instance, MUST include a RECURRENCE-ID property indicating which instance they refer to.

The returned calendar components MUST NOT use recurrence properties (i.e., EXDATE, EXRULE, RDATE, and RRULE) and MUST NOT have reference to or include VTIMEZONE components. Date and local time with reference to time zone information MUST be converted into date with UTC time.

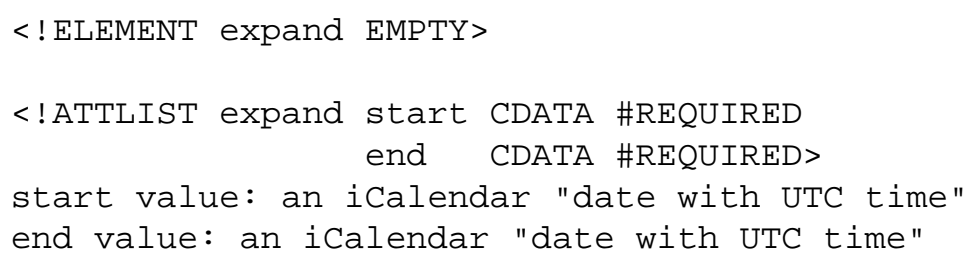

\subsubsection{CALDAV:limit-recurrence-set XML Element}

Name:

Namespace:

Purpose:

Description:

\author{
limit-recurrence-set
}

urn:ietf:params:xml:ns:caldav

Specifies a time range to limit the set of "overridden components" returned by the server.

The CALDAV:limit-recurrence-set XML element specifies that for a given calendaring REPORT request, the server MUST return, in addition to the "master component", only the "overridden components" that impact a specified time range. An overridden component impacts a time range if its current start and end times overlap the time range, or if the original start and end times -- the ones that would have been used if the instance were not overridden -- overlap the time range.

The "start" attribute specifies the inclusive start of the time range, and the "end" attribute specifies the non-inclusive end of the time range. Both attributes are specified as date with UTC time value. The value of the "end" attribute MUST be greater than the value of the "start" attribute.

The server MUST use the same logic as defined for CALDAV:time-range to determine if the current or original scheduled time of an "overridden" recurrence instance intersects the specified time range.

Overridden components that have a RANGE parameter on their RECURRENCEID property may specify one or more instances in the recurrence set, and some of those instances may fall within the specified time range or may have originally fallen within the specified time range prior to being overridden. If that is the case, the 
Definition:

overridden component MUST be included in the results, as it has a direct impact on the interpretation of instances within the specified time range.

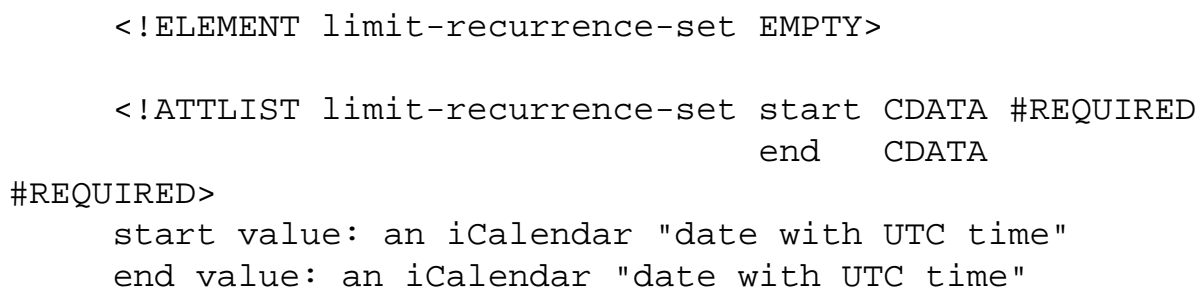

\subsubsection{CALDAV:limit-freebusy-set XML Element}

Name:

Namespace:

Purpose:

Description:

Definition: limit-freebusy-set

urn:ietf:params:xml:ns:caldav

Specifies a time range to limit the set of FREEBUSY values returned by the server.

The CALDAV:limit-freebusy-set XML element specifies that for a given calendaring REPORT request, the server MUST only return the FREEBUSY property values of a VFREEBUSY component that intersects a specified time range.

The "start" attribute specifies the inclusive start of the time range, and the "end" attribute specifies the non-inclusive end of the time range. Both attributes are specified as "date with UTC time" value. The value of the "end" attribute MUST be greater than the value of the "start" attribute.

The server MUST use the same logic as defined for CALDAV:time-range to determine if a FREEBUSY property value intersects the specified time range.

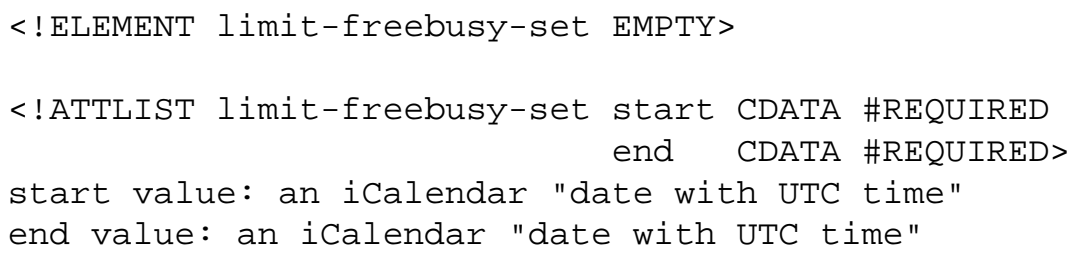

\subsection{CALDAV:filter XML Element}

Name:

Namespace:

Purpose:

Description:

Definition: filter

urn:ietf:params:xml:ns:caldav

Specifies a filter to limit the set of calendar components returned by the server.

The CALDAV:filter XML element specifies the search filter used to limit the calendar components returned by a calendaring REPORT request.

$<$ !ELEMENT filter (comp-filter) >

\subsubsection{CALDAV:comp-filter XML Element}

Name:

Namespace:

Purpose:

Description: comp-filter

urn:ietf:params:xml:ns:caldav

Specifies search criteria on calendar components.

The CALDAV:comp-filter XML element specifies a query targeted at the calendar object (i.e., VCALENDAR) or at a specific calendar component type (e.g., VEVENT). 
Definition:

The scope of the CALDAV:comp-filter XML element is the calendar object when used as a child of the CALDAV:filter XML element. The scope of the CALDAV:comp-filter XML element is the enclosing calendar component when used as a child of another CALDAV:comp-filter XML element. A CALDAV:comp-filter is said to match if:

- The CALDAV:comp-filter XML element is empty and the calendar object or calendar component type specified by the "name" attribute exists in the current scope;

or:

- The CALDAV:comp-filter XML element contains a CALDAV:is-not-defined XML element and the calendar object or calendar component type specified by the "name" attribute does not exist in the current scope;

or:

- The CALDAV:comp-filter XML element contains a CALDAV:time-range XML element and at least one recurrence instance in the targeted calendar component is scheduled to overlap the specified time range, and all specified CALDAV:propfilter and CALDAV:comp-filter child XML elements also match the targeted calendar component;

or:

- The CALDAV:comp-filter XML element only contains CALDAV:prop-filter and CALDAV:comp-filter child XML elements that all match the targeted calendar component.

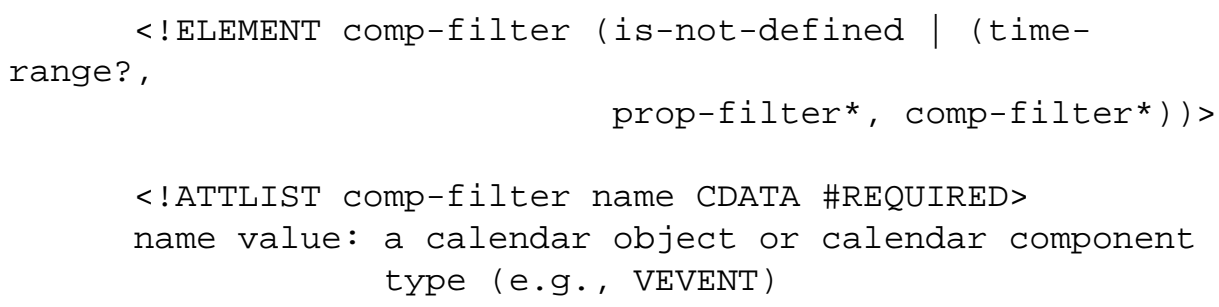

\subsubsection{CALDAV:prop-filter XML Element}

Name:

Namespace:

Purpose:

Description: prop-filter

urn:ietf:params:xml:ns:caldav

Specifies search criteria on calendar properties.

The CALDAV:prop-filter XML element specifies a query targeted at a specific calendar property (e.g., CATEGORIES) in the scope of the enclosing calendar component. A calendar property is said to match a CALDAV:prop-filter if:

- The CALDAV:prop-filter XML element is empty and a property of the type specified by the "name" attribute exists in the enclosing calendar component;

or:

- The CALDAV:prop-filter XML element contains a CALDAV:is-not-defined XML element and no property of the type specified by the "name" attribute exists in the enclosing calendar component;

or:

- The CALDAV:prop-filter XML element contains a CALDAV:time-range XML element and the property value overlaps the specified time range, and all specified CALDAV:param-filter child XML elements also match the targeted property; 
or:

- The CALDAV:prop-filter XML element contains a CALDAV:text-match XML element and the property value matches it, and all specified CALDAV:param-filter child XML elements also match the targeted property;

Definition:

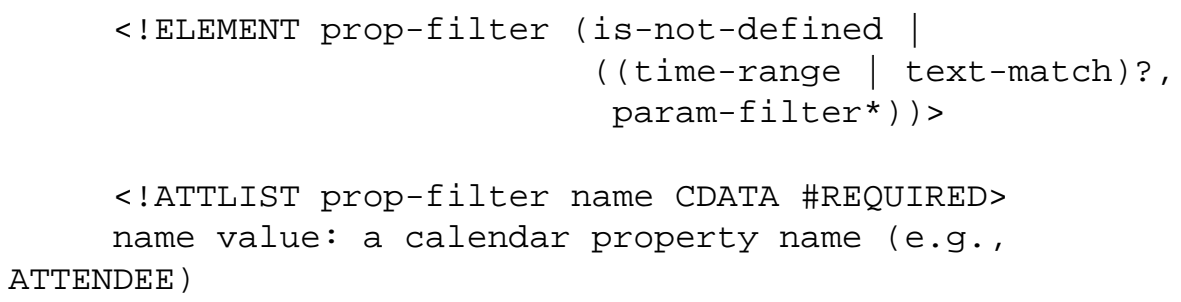

\subsubsection{CALDAV:param-filter XML Element}

Name:

Namespace:

Purpose:

Description:

Definition: param-filter

urn:ietf:params:xml:ns:caldav

Limits the search to specific parameter values.

The CALDAV:param-filter XML element specifies a query targeted at a specific calendar property parameter (e.g., PARTSTAT) in the scope of the calendar property on which it is defined. A calendar property parameter is said to match a CALDAV:param-filter if:

- The CALDAV:param-filter XML element is empty and a parameter of the type specified by the "name" attribute exists on the calendar property being examined;

or:

- The CALDAV:param-filter XML element contains a CALDAV:is-not-defined XML element and no parameter of the type specified by the "name" attribute exists on the calendar property being examined;

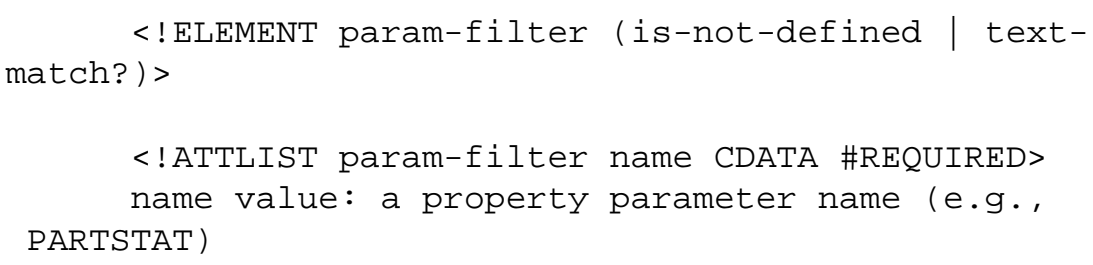

\subsubsection{CALDAV:is-not-defined XML Element}

Name:

is-not-defined

Namespace:

urn:ietf:params:xml:ns:caldav

Purpose:

Description:

Specifies that a match should occur if the enclosing component, property, or parameter does not exist.

The CALDAV:is-not-defined XML element specifies that a match occurs if the enclosing component, property, or parameter value specified in a calendaring REPORT request does not exist in the calendar data being tested.

Definition: 


\subsubsection{CALDAV:text-match XML Element}

Name:

Namespace:

Purpose:

Description:

Definition:

text-match

urn:ietf:params:xml:ns:caldav

Specifies a substring match on a property or parameter value.

The CALDAV:text-match XML element specifies text used for a substring match against the property or parameter value specified in a calendaring REPORT request.

The "collation" attribute is used to select the collation that the server MUST use for character string matching. In the absence of this attribute, the server MUST use the "i;ascii-casemap" collation.

The "negate-condition" attribute is used to indicate that this test returns a match if the text matches when the attribute value is set to "no", or return a match if the text does not match, if the attribute value is set to "yes". For example, this can be used to match components with a STATUS property not set to CANCELLED.

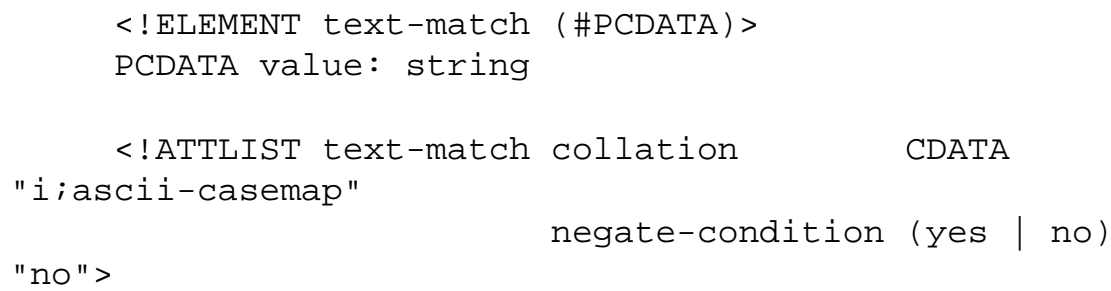

\subsection{CALDAV:timezone XML Element}

Name:

Namespace:

Purpose:

Description:

Note:

Definition: timezone

urn:ietf:params:xml:ns:caldav

Specifies the time zone component to use when determining the results of a report.

The CALDAV:timezone XML element specifies that for a given calendaring REPORT request, the server MUST rely on the specified VTIMEZONE component instead of the CALDAV:calendar-timezone property of the calendar collection, in which the calendar object resource is contained to resolve "date" values and "date with local time" values (i.e., floating time) to "date with UTC time" values. The server will require this information to determine if a calendar component scheduled with "date" values or "date with local time" values intersects a CALDAV:time-range specified in a CALDAV:calendar-query REPORT.

The iCalendar data embedded within the CALDAV:timezone XML element MUST follow the standard XML character data encoding rules, including use of \&lt; \&gt; \&amp; etc. entity encoding or the use of a $<$ ![CDATA $[. .].]>$ construct. In the later case, the iCalendar data cannot contain the character sequence "]]>", which is the end delimiter for the CDATA section.

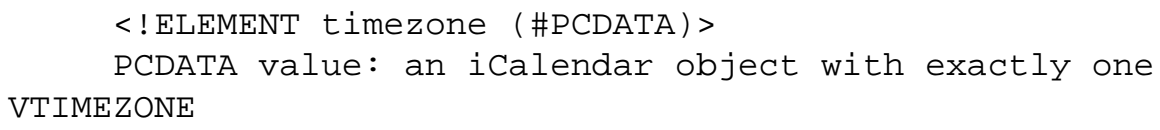




\subsection{CALDAV:time-range XML Element}

Name:

Namespace:

Purpose:

Description: time-range

urn:ietf:params:xml:ns:caldav

Specifies a time range to limit the set of calendar components returned by the server.

The CALDAV:time-range XML element specifies that for a given calendaring REPORT request, the server MUST only return the calendar object resources that, depending on the context, have a component or property whose value intersects a specified time range.

The "start" attribute specifies the inclusive start of the time range, and the "end" attribute specifies the non-inclusive end of the time range. Both attributes MUST be specified as "date with UTC time" value. Time ranges open at one end can be specified by including only one attribute; however, at least one attribute MUST always be present in the CALDAV:time-range element. If either the "start" or "end" attribute is not specified in the CALDAV:time-range XML element, assume "-infinity" and "+infinity" as their value, respectively. If both "start" and "end" are present, the value of the "end" attribute MUST be greater than the value of the "start" attribute.

Time range tests MUST consider every recurrence instance when testing the time range condition; if any one instance matches, then the test returns true. Testing recurrence instances requires the server to infer an effective value for DTSTART, DTEND, DURATION, and DUE properties for an instance based on the recurrence patterns and any overrides.

A VEVENT component overlaps a given time range if the condition for the corresponding component state specified in the table below is satisfied. Note that, as specified in [RFC2445], the DTSTART property is REQUIRED in the VEVENT component. The conditions depend on the presence of the DTEND and DURATION properties in the VEVENT component. Furthermore, the value of the DTEND property MUST be later in time than the value of the DTSTART property. The duration of a VEVENT component with no DTEND and DURATION properties is 1 day (+P1D) 
when the DTSTART is a DATE value, and 0 seconds when the DTSTART is a DATETIME value.

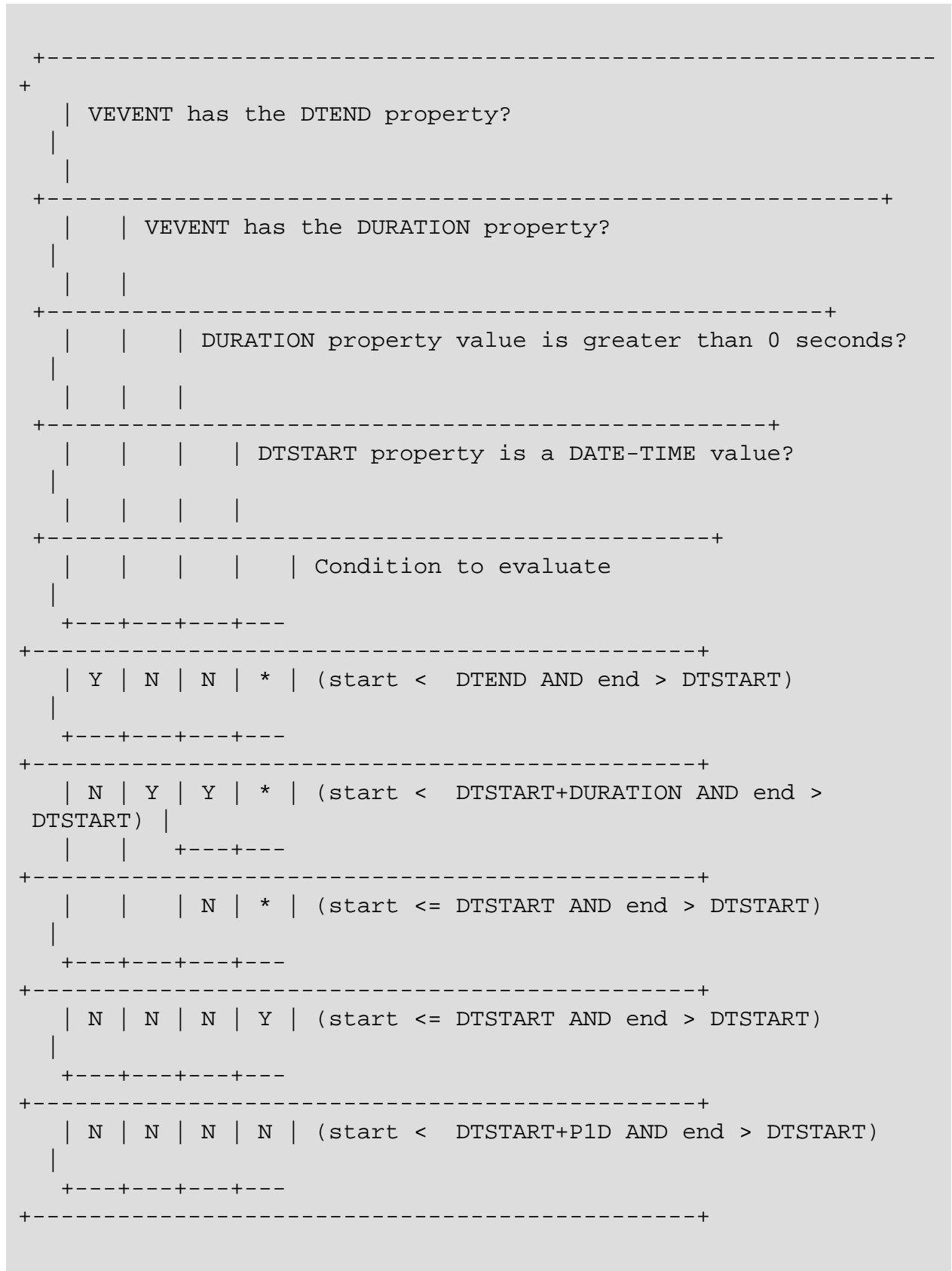

A VTODO component is said to overlap a given time range if the condition for the corresponding component state specified in the table below is satisfied. The conditions depend on the presence of the DTSTART, DURATION, DUE, COMPLETED, and CREATED properties in the VTODO component. Note that, as specified in 
[RFC2445], the DUE value MUST be a DATE-TIME value equal to or after the DTSTART value if specified. 


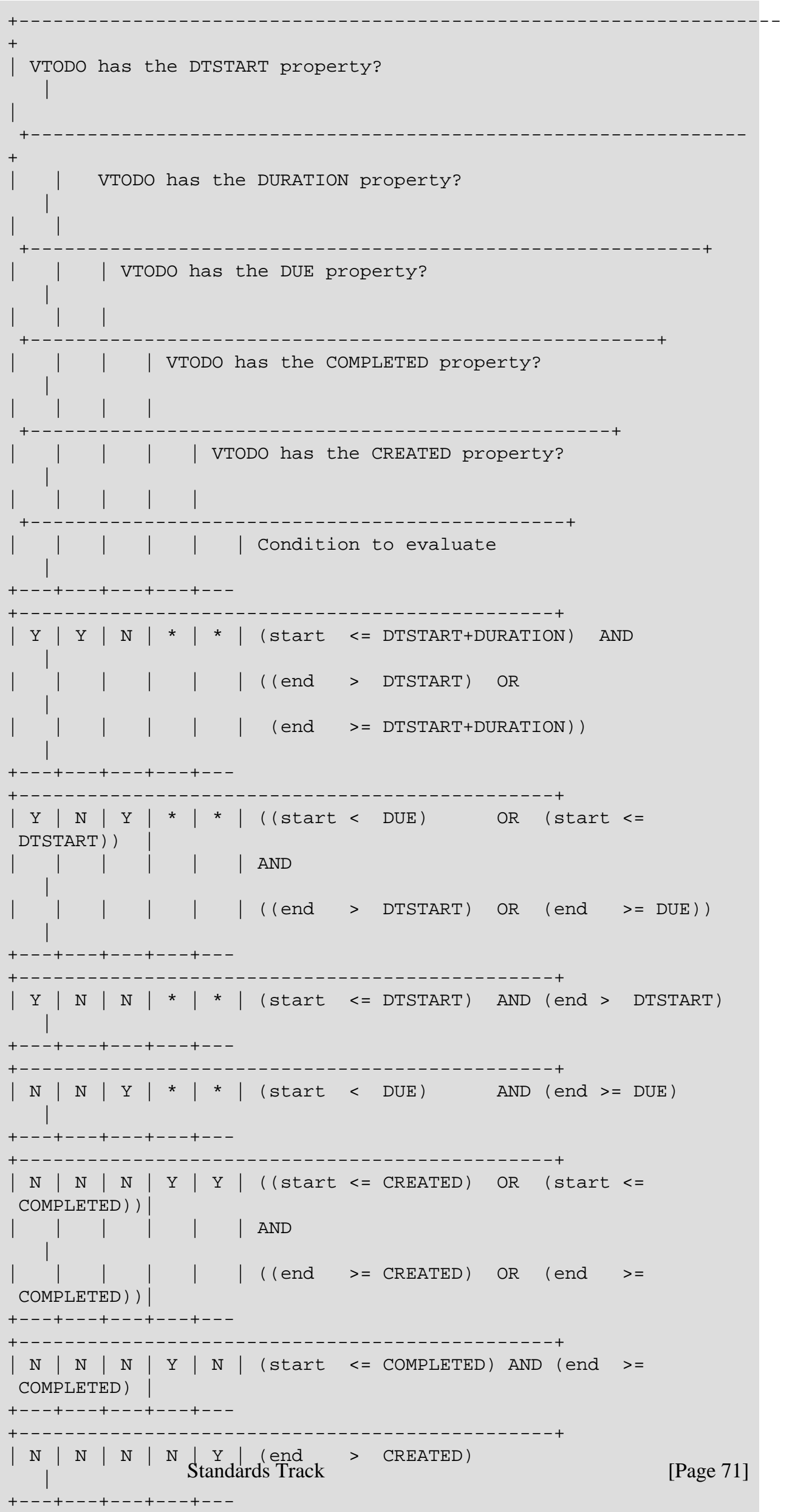

Daboo, et al. 
A VJOURNAL component overlaps a given time range if the condition for the corresponding component state specified in the table below is satisfied. The conditions depend on the presence of the DTSTART property in the VJOURNAL component and on whether the DTSTART is a DATE-TIME or DATE value. The effective "duration" of a VJOURNAL component is 1 day (+P1D) when the DTSTART is a DATE value, and 0 seconds when the DTSTART is a DATE-TIME value.

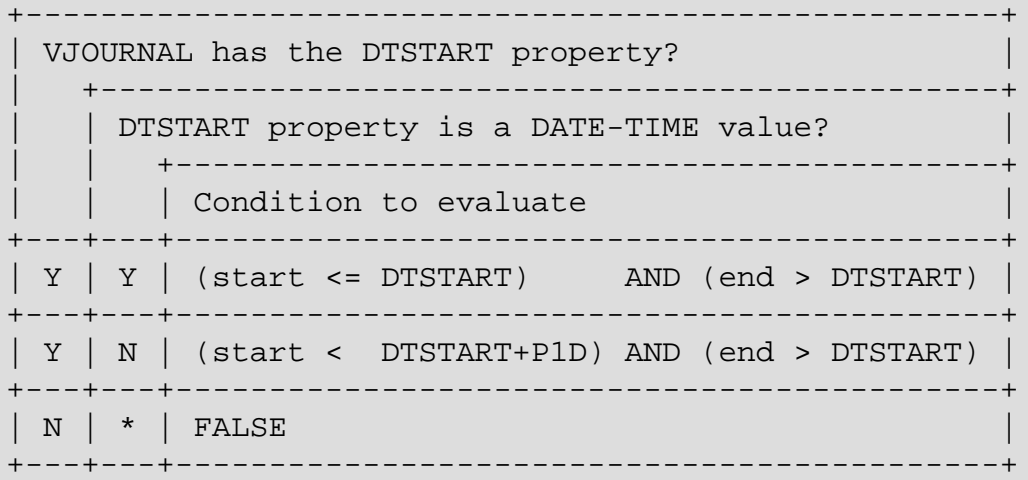

A VFREEBUSY component overlaps a given time range if the condition for the corresponding component state specified in the table below is satisfied. The conditions depend on the presence in the VFREEBUSY component of the DTSTART and DTEND properties, and any FREEBUSY properties in the absence of DTSTART and DTEND. Any DURATION property is ignored, as it has a special meaning when used in a VFREEBUSY component.

When only FREEBUSY properties are used, each period in each FREEBUSY property is compared against the time range, irrespective of the type of free busy information (free, busy, busy-tentative, busy-unavailable) represented by the property.

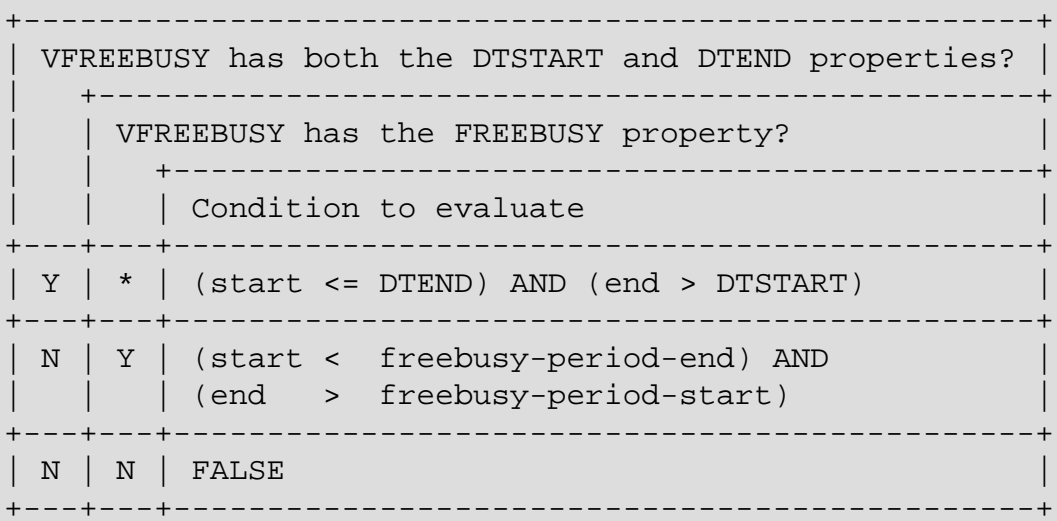

A VALARM component is said to overlap a given time range if the following condition holds:

(start <= trigger-time) AND (end > trigger-time)

A VALARM component can be defined such that it triggers repeatedly. Such a VALARM component is said to overlap a given time range if at least one of its triggers overlaps the time range. 
Definition:

The calendar properties COMPLETED, CREATED, DTEND, DTSTAMP, DTSTART, DUE, and LAST-MODIFIED overlap a given time range if the following condition holds:

(start <= date-time) AND (end > date-time)

Note that if DTEND is not present in a VEVENT, but DURATION is, then the test should instead operate on the 'effective' DTEND, i.e., DTSTART+DURATION. Similarly, if DUE is not present in a VTODO, but DTSTART and DURATION are, then the test should instead operate on the 'effective' DUE, i.e., DTSTART + DURATION.

The semantic of CALDAV:time-range is not defined for any other calendar components and properties.

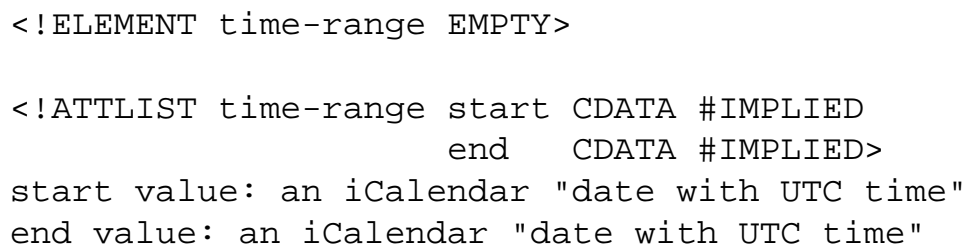

\subsection{CALDAV:calendar-multiget XML Element}

Name:

Namespace:

Purpose:

Description:

Definition: calendar-multiget

urn:ietf:params:xml:ns:caldav

CalDAV report used to retrieve specific calendar object resources.

See Section 7.9.

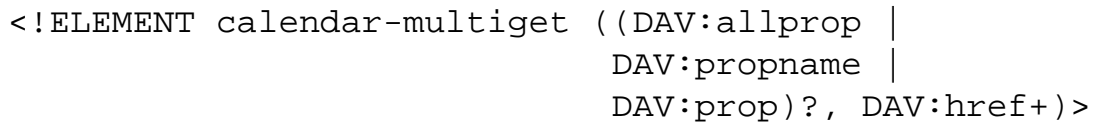

\subsection{CALDAV:free-busy-query XML Element}

Name:

Namespace:

Purpose:

Description:

Definition: free-busy-query

urn:ietf:params:xml:ns:caldav

CalDAV report used to generate a VFREEBUSY to determine busy time over a specific time range.

See Section 7.10.

$<$ !ELEMENT free-busy-query (time-range) $>$ 


\section{Internationalization Considerations}

CalDAV allows internationalized strings to be stored and retrieved for the description of calendar collections (see Section 5.2.1).

The CALDAV:calendar-query REPORT (Section 7.8) includes a text searching option controlled by the CALDAV:text-match element, and details of character handling are covered in the description of that element (see Section 9.7.5). 


\section{Security Considerations}

HTTP protocol transactions are sent in the clear over the network unless protection from snooping is negotiated. This can be accomplished by use of TLS, as defined in [RFC2818]. In particular, HTTP Basic authentication MUST NOT be used unless TLS is in effect.

Servers MUST take adequate precautions to ensure that malicious clients cannot consume excessive server resources (CPU, memory, disk, etc.) through carefully crafted reports. For example, a client could upload an event with a recurrence rule that specifies a recurring event occurring every second for the next 100 years, which would result in approximately $3 \times 10^{\wedge} 9$ instances! A report that asks for recurrences to be expanded over that range would likely constitute a denial-of-service attack on the server.

When creating new resources (including calendar collections), clients MUST ensure that the resource name (the last path segment of the resource URI) assigned to the new resource does not expose any data from within the iCalendar resource itself or information about the nature of a calendar collection. This is required to ensure that the presence of a specific iCalendar component or nature of components in a collection cannot be inferred based on the name of a resource.

When rolling up free-busy information, more information about a user's events is exposed if busy periods overlap or are adjacent (this tells the client requesting the free-busy information that the calendar owner has at least two events, rather than knowing only that the calendar owner has one or more events during the busy period). Thus, a conservative approach to calendar data privacy would have servers always coalesce such busy periods when they are the same type.

Procedure alarms are a known security risk for either clients or servers to handle, particularly when the alarm was created by another agent. Clients and servers are not required to execute such procedure alarms.

Security considerations described in iCalendar [RFC2445] and iTIP [RFC2446] are also applicable to CalDAV. Beyond these, CalDAV does not raise any security considerations that are not present in HTTP [RFC2616] and WebDAV [RFC2518], [RFC3253], [RFC3744]. 


\section{IANA Considerations}

This document uses one new URN to identify a new XML namespace. The URN conforms to a registry mechanism described in [RFC3688].

\subsection{Namespace Registration}

Registration request for the CalDAV namespace:

URI: urn:ietf:params:xml:ns:caldav

Registrant Contact: See the "Authors' Addresses" section of this document.

XML: None. Namespace URIs do not represent an XML specification. 


\section{Acknowledgements}

The authors would like to thank the following individuals for contributing their ideas and support for writing this specification: Michael Arick, Mario Bonin, Chris Bryant, Scott Carr, Andre Courtemanche, Mike Douglass, Ted Hardie, Marten den Haring, Jeffrey Harris, Sam Hartman, Helge Hess, Jeff McCullough, Alexey Melnikov, Dan Mosedale, Brian Moseley, Francois Perrault, Kervin L. Pierre, Julian F. Reschke, Wilfredo Sanchez Vega, Mike Shaver, Jari Urpalainen, Simon Vaillancourt, and Jim Whitehead.

The authors would also like to thank the Calendaring and Scheduling Consortium for advice with this specification, and for organizing interoperability testing events to help refine it. 


\section{References}

\subsection{Normative References}

[RFC2119]

[RFC2246]

[RFC2445]

[RFC2446]

[RFC2518]

[RFC2616]

[RFC2818]

[RFC3253]

[RFC3688]

[RFC3744]

[RFC4346]

[RFC4790]

[W3C.REC-xml-20060816]
Bradner, S., "Key words for use in RFCs to Indicate Requirement Levels", BCP 14, RFC 2119, March 1997.

Dierks, T. and C. Allen, "The TLS Protocol Version 1.0", RFC 2246, January 1999.

Dawson, F., Stenerson, D., "Internet Calendaring and Scheduling Core Object Specification (iCalendar)", RFC 2445, November 1998.

Silverberg, S., Mansour, S., Dawson, F., and R. Hopson, "iCalendar Transport-Independent Interoperability Protocol (iTIP) Scheduling Events, BusyTime, To-dos and Journal Entries", RFC 2446, November 1998.

Goland, Y., Whitehead, E., Faizi, A., Carter, S., and D. Jensen, "HTTP Extensions for Distributed Authoring -- WEBDAV", RFC 2518, February 1999.

Fielding, R., Gettys, J., Mogul, J., Frystyk, H., Masinter, L., Leach, P., and T. Berners-Lee, "Hypertext Transfer Protocol -- HTTP/1.1", RFC 2616, June 1999.

Rescorla, E., "HTTP Over TLS", RFC 2818, May 2000.

Clemm, G., Amsden, J., Ellison, T., Kaler, C., and J. Whitehead, "Versioning Extensions to WebDAV (Web Distributed Authoring and Versioning)", RFC 3253, March 2002.

Mealling, M., "The IETF XML Registry", BCP 81, RFC 3688, January 2004.

Clemm, G., Reschke, J., Sedlar, E., and J. Whitehead, "Web Distributed Authoring and Versioning (WebDAV) Access Control Protocol", RFC 3744, May 2004.

Dierks, T. and E. Rescorla, "The Transport Layer Security (TLS) Protocol Version 1.1", RFC 4346, April 2006.

Newman, C., Duerst, M., and A. Gulbrandsen, "Internet Application Protocol Collation Registry", RFC 4790, March 2007.

Paoli, J., Maler, E., Yergeau, F., Sperberg-McQueen, C., and T. Bray, "Extensible Markup Language (XML) 1.0 (Fourth Edition)", World Wide Web Consortium Recommendation REC-xml-20060816, August 2006, <http://www.w3.org/TR/2006/REC-xml-20060816>.

\subsection{Informative References}

[RFC2426]

[rfc2518bis]

[RFC2739]

[RFC4331]
Dawson, F. and T. Howes, "vCard MIME Directory Profile", RFC 2426, September 1998.

Dusseault, L., "HTTP Extensions for Distributed Authoring - WebDAV", Work in Progress, December 2006.

Small, T., Hennessy, D., and F. Dawson, "Calendar Attributes for vCard and LDAP", RFC 2739, January 2000.

Korver, B. and L. Dusseault, "Quota and Size Properties for Distributed Authoring and Versioning (DAV) Collections", RFC 4331, February 2006. 

Protocol", RFC 4511, June 2006. 


\section{Appendix A. CalDAV Method Privilege Table (Normative)}

The following table extends the WebDAV Method Privilege Table specified in Appendix B of [RFC3744].

\begin{tabular}{|l|l|}
\hline METHOD & PRIVILEGES \\
\hline MKCALENDAR & DAV:bind \\
REPORT & $\begin{array}{l}\text { DAV:read or CALDAV:read-free-busy (on all } \\
\text { referenced resources) }\end{array}$ \\
\hline
\end{tabular}




\section{Appendix B. Calendar Collections Used in the Examples}

This appendix shows the calendar object resources contained in the calendar collection queried in the examples throughout this document.

The content of the calendar collection is being shown as if it were returned by a CALDAV:calendar-query REPORT request designed to return all the calendar data in the collection:

> Request $<<$

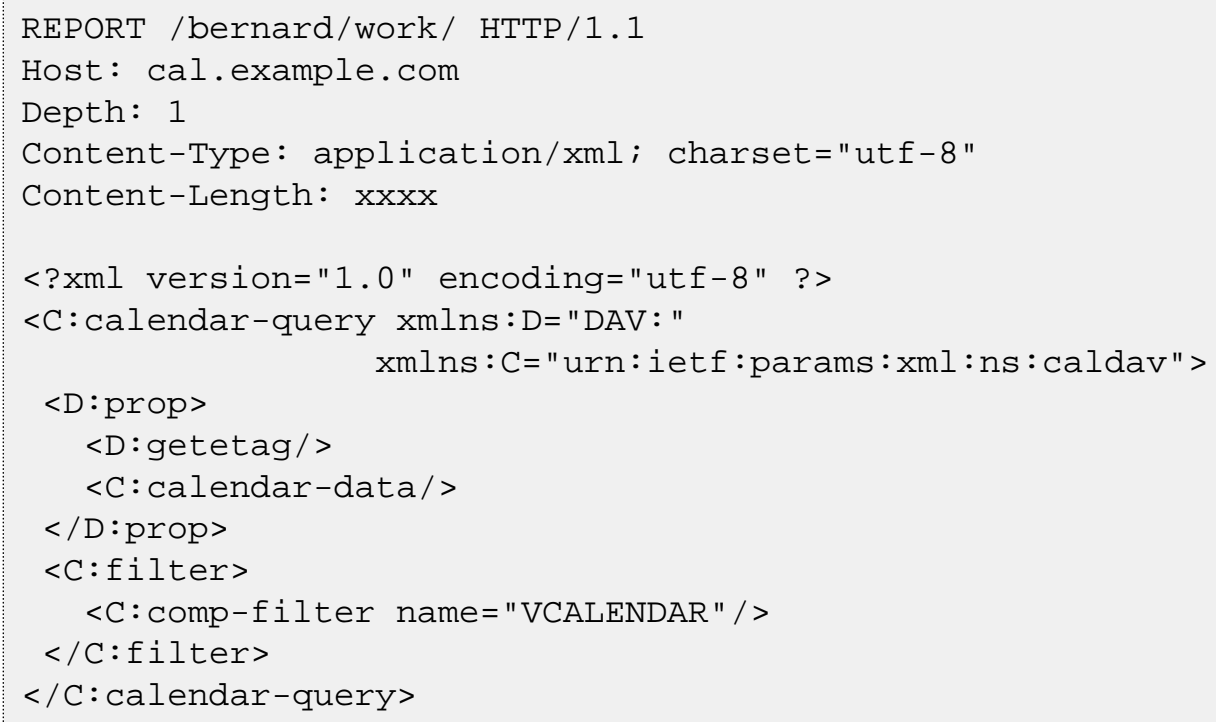




\section{$>$ Response $<<$}

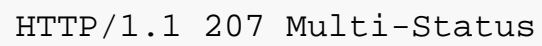

DabogRetat.D : - / / Example Corp. / / CalDAStamdards TyackN 


\section{Index}

\section{C}

CALDAV:calendar-collection-location-ok precondition 18

CALDAV:calendar-description property $\mathbf{1 1}$

CALDAV:calendar-home-set property $\mathbf{2 3}$

CALDAV:calendar-multiget report $\mathbf{4 8}$

CALDAV:calendar-query report $\mathbf{2 8}$

CALDAV:calendar-timezone property $\mathbf{1 2}$

CALDAV:free-busy-query report $\mathbf{5 2}$

CALDAV:max-attendees-per-instance property $\mathbf{1 6}$

CALDAV:max-date-time precondition 29, 49

CALDAV:max-date-time property $\mathbf{1 5}$

CALDAV:max-instances property $\mathbf{1 6}$

CALDAV:max-resource-size property $\mathbf{1 4}$

CALDAV:min-date-time precondition 28, 49

CALDAV:min-date-time property $\mathbf{1 5}$

CALDAV:read-free-busy $\mathbf{2 3}$

CALDAV:supported-calendar-component-set property $\mathbf{1 3}$

CALDAV:supported-calendar-data precondition 28, 49

CALDAV:supported-calendar-data property 14

CALDAV:supported-collation precondition $\mathbf{2 9}$

CALDAV:supported-filter precondition $\mathbf{2 8}$

CALDAV:valid-calendar-data precondition 18, 28

CALDAV:valid-filter precondition $\mathbf{2 8}$

Condition Names

CALDAV:calendar-collection-location-ok (pre) $\mathbf{1 8}$

CALDAV:max-date-time (pre) 29, 49

CALDAV:min-date-time (pre) 28, 49

CALDAV:supported-calendar-data (pre) 28, 49

CALDAV:supported-collation (pre) 29

CALDAV:supported-filter (pre) 28

CALDAV:valid-calendar-data (pre) 18, 28

CALDAV:valid-filter (pre) 28

DAV:needs-privilege (pre) 18

DAV:number-of-matches-within-limits (post) 29, 53

DAV:resource-must-be-null (pre) 18

\section{D}

DAV:needs-privilege precondition $\mathbf{1 8}$

DAV:number-of-matches-within-limits postcondition 29, 53

DAV:resource-must-be-null precondition $\mathbf{1 8}$

M

Methods

MKCALENDAR 17

MKCALENDAR method 17

$\mathbf{P}$

Privileges

CALDAV:read-free-busy 23

Properties

CALDAV:calendar-description 11

CALDAV:calendar-home-set $\mathbf{2 3}$

CALDAV:calendar-timezone $\mathbf{1 2}$

CALDAV:max-attendees-per-instance $\mathbf{1 6}$

CALDAV:max-date-time $\mathbf{1 5}$

CALDAV:max-instances $\mathbf{1 6}$

CALDAV:max-resource-size $\mathbf{1 4}$
CALDAV:min-date-time $\mathbf{1 5}$

CALDAV:supported-calendar-component-set $\mathbf{1 3}$

CALDAV:supported-calendar-data 14

$\mathbf{R}$

Reports

CALDAV:calendar-multiget $\mathbf{4 8}$

CALDAV:calendar-query $\mathbf{2 8}$

CALDAV:free-busy-query $\mathbf{5 2}$

RFC 2119 5, 78

$R F C 2246$ 6, 6, 78

$R F C 2426$ 56, 78

RFC2445 5, 6, 20, 22, 26, 57, 68, 70, 75, 78

Section 3.1020

Section 4.222

RFC2446 75, 78

RFC 2518 5, 5, 5, 5, 6, 6, 11, 12, 13, 14, 14, 15, 15, 16, 16, $17,23,26,61,62,62,75,78$

Section 12.13.2 17

Section 12.14.1 11, 12, 13, 14, 14, 15, 15, 16, 16, 23, 26

Section 145

rfc2518bis 6, 78

$R F C 2616$ 5, 6, 75, 78

RFC2739 56, 78

RFC2818 6, 75, 78

RFC3253 5, 6, 25, 25, 25, 75, 78

Section 1.4.2 5

Section 3.1.5 25

Section 3.625

Section 3.825

RFC3688 76, 78

$R F C 3744$ 6, 23, 23, 56, 75, 78, 80

RFC4331 57, 78

$R F C 4346$ 6, 78

RFC4511 56, 79

$R F C 4790$ 26, 26, 26, 26, 26, 59,78

Section 3.126

Section 3.226

Section 4.226

W

W3C.REC-xml-20060816 5, 78

Section 3.25 


\section{Authors' Addresses}

Cyrus Daboo

Apple Inc.

1 Infinite Loop

Cupertino, CA 95014

USA

Email: cyrus@ daboo.name

URI: http://www.apple.com/

\section{Bernard Desruisseaux}

Oracle Corporation

600 Blvd. de Maisonneuve West

Suite 1900

Montreal, QC H3A 3J2

CANADA

Email: bernard.desruisseaux@oracle.com

URI: http://www.oracle.com/

\section{Lisa Dusseault}

CommerceNet

169 University Ave.

Palo Alto, CA 94301

USA

Email:1dusseault@commerce.net

URI: http://commerce.net/

\section{Full Copyright Statement}

Copyright $\odot$ The IETF Trust (2007).

This document is subject to the rights, licenses and restrictions contained in BCP 78, and except as set forth therein, the authors retain all their rights.

This document and the information contained herein are provided on an "AS IS" basis and THE CONTRIBUTOR, THE ORGANIZATION HE/SHE REPRESENTS OR IS SPONSORED BY (IF ANY), THE INTERNET SOCIETY, THE IETF TRUST AND THE INTERNET ENGINEERING TASK FORCE DISCLAIM ALL WARRANTIES, EXPRESS OR IMPLIED, INCLUDING BUT NOT LIMITED TO ANY WARRANTY THAT THE USE OF THE INFORMATION HEREIN WILL NOT INFRINGE ANY RIGHTS OR ANY IMPLIED WARRANTIES OF MERCHANTABILITY OR FITNESS FOR A PARTICULAR PURPOSE.

\section{Intellectual Property}

The IETF takes no position regarding the validity or scope of any Intellectual Property Rights or other rights that might be claimed to pertain to the implementation or use of the technology described in this document or the extent to which any license under such rights might or might not be available; nor does it represent that it has made any independent effort to identify any such rights. Information on the procedures with respect to rights in RFC documents can be found in BCP 78 and BCP 79.

Copies of IPR disclosures made to the IETF Secretariat and any assurances of licenses to be made available, or the result of an attempt made to obtain a general license or permission for the use of such proprietary rights by implementers or users of this specification can be obtained from the IETF on-line IPR repository at http://www .ietf.org/ipr ${ }^{1}$.

\footnotetext{
${ }^{1}$ http://www.ietf.org/ipr
} 
The IETF invites any interested party to bring to its attention any copyrights, patents or patent applications, or other proprietary rights that may cover technology that may be required to implement this standard. Please address the information to the IETF at ietf-ipr@ietf.org ${ }^{2}$.

\section{Acknowledgement}

Funding for the RFC Editor function is provided by the IETF Administrative Support Activity (IASA).

2 mailto:ietf-ipr@ietf.org 